\title{
Representations of Algebraic Quantum Groups and Reconstruction Theorems for Tensor Categories
}

\author{
M. Müger, J. E. Roberts and L. Tuset
}

October 24,2018

\begin{abstract}
We give a pedagogical survey of those aspects of the abstract representation theory of quantum groups which are related to the Tannaka-Krein reconstruction problem. We show that every concrete semisimple tensor *-category with conjugates is equivalent to the category of finite dimensional non-degenerate *representations of a discrete algebraic quantum group. Working in the self-dual framework of algebraic quantum groups, we then relate this to earlier results of S. L. Woronowicz and S. Yamagami. We establish the relation between braidings and $R$-matrices in this context. Our approach emphasizes the role of the natural transformations of the embedding functor. Thanks to the semisimplicity of our categories and the emphasis on representations rather than corepresentations, our proof is more direct and conceptual than previous reconstructions. As a special case, we reprove the classical Tannaka-Krein result for compact groups. It is only here that analytic aspects enter, otherwise we proceed in a purely algebraic way. In particular, the existence of a Haar functional is reduced to a well known general result concerning discrete multiplier Hopf *-algebras.
\end{abstract}

\section{Introduction}

Pontryagin's duality theory for locally compact abelian groups and the Tannaka-Krein theory for compact groups are two major results in the theory of harmonic analysis on topological groups 14. The Pontryagin duality theorem is a statement concerning characters, whereas the Tannaka-Krein theorem is a statement involving irreducible unitary representations. These two notions coincide whenever the group is both abelian and compact.

Pontryagin's theorem can be stated more generally for locally compact quantum groups [21], a notion evolving out of Kac algebras [1]] and compact matrix pseudogroups 440].

The theory of representations is most naturally developed in the language of tensor categories [24]. The category of finite dimensional representations of a compact group is a symmetric tensor $*$-category with conjugates [9], and the Tannaka-Krein theorem tells us how to reconstruct the group from the latter. In 1988, starting from a tensor $*$-category [12 with conjugates and admitting a generator, but assuming neither a symmetry nor a braiding, S. L. Woronowicz reconstructed [41] a compact matrix pseudogroup [40] having the given category as its category of finite dimensional unitary corepresentations. No general definition of a compact quantum group existed at the time. Once it did, his proof generalizes to categories without generator. Alternatively, one can use the fact that every $*$-category and every compact quantum group are inductive limits of categories with generators and of compact matrix pseudogroups, respectively.

For the Tannaka-Krein results mentioned so far, the starting point is a concrete category, i.e. a (non-full) subcategory of the tensor category of Hilbert spaces. It is conceptually more satisfactory to start from an abstract tensor category together with a faithful tensor functor into the category of Hilbert spaces. In the work of N. Saavedra Rivano [31] the group associated with a concrete symmetric tensor category was identified as the group of natural monoidal automorphisms of the embedding functor as Tannaka had effectively done [33] before the advent of category theory. The idea of considering the natural transformations of the embedding functor was generalized in the work of K.-H. Ulbrich [34], where a given concrete tensor category is identified as the category of comodules over a Hopf algebra, cf. also [45]. 
While the role of the natural transformations of the embedding functor is obscure in Woronowicz's approach, they appear at least implicitly in the work of S. Yamagami [43], who considered representations of discrete quantum groups. His approach has two drawbacks. On the one hand, he assumes that the category is equipped with an additional piece of structure, an ' $\varepsilon$-structure'. A very similar notion naturally arises in recent axiomatizations of tensor categories with two-sided duals [2], but it is quite superfluous if one works with $*$-categories. This will become clear in our treatment. (Yamagami has recently proved a result [44, Theorem 3.6] implying that, passing if necessary to an equivalent tensor $*$-category, there is an essentially unique $\varepsilon$-structure.) On the other hand, the von Neumann algebraic formulation of discrete quantum groups used in [43] is considerably more involved than current definitions, in that it unnecessarily mixes analytic and algebraic structures.

In neither of 40, 43 does one find a complete proof of equivalence between the given category and the representation category of the derived quantum group. Also questions of uniqueness and braidings have not been addressed.

It is well known that the self-duality of the category of finite dimensional Hopf algebras breaks down in the infinite dimensional case. Motivated by the desire to find a purely algebraic framework for quantum groups admitting a version of Pontryagin duality, A. Van Daele developed the theory of algebraic quantum groups (aqg) [37]. This is achieved by admitting non-unital algebras and requiring the existence of a positive and leftinvariant functional, the Haar functional. All compact and all discrete quantum groups are aqg, and every aqg has a unique analytic extension to a locally compact quantum group [20], having an equivalent von Neumann algebraic version [22]. A discrete multiplier Hopf *-algebra [35] can be shown to have a Haar functional rendering it a discrete aqg.

The purpose of the present paper is to give a coherent and reasonably complete survey of the TannakaKrein theory of quantum groups. The only other review we are aware of is 15, which appeared ten years ago. At the time, no appropriate self-dual category of quantum groups existed. (In the same year, P. Podlés and Woronowicz, in defining a discrete quantum group, took the first step in this direction [28].) The approach to Tannakian categories motivated by algebraic geometry is well reviewed in [6], where, however, only symmetric tensor categories are considered.

Our approach to generalized Tannaka-Krein theory adopts the philosophy on quantum groups in [41, 43], meaning a self-dual category with emphasis on $*$-categories. In contradistinction to these authors we wish to distinguish categorical from quantum group aspects as well as algebraic from analytic aspects as far as possible. Following [31, 7, 34, 45], we emphasize natural transformations of the embedding functor. Yet, our use of natural transformations is more direct and we work with representations rather than corepresentations. The algebra product is just the composition of natural transformations and the coproduct is defined directly in terms of the tensor structure, the tensor unit giving rise to the counit. This yields a quantum semigroup, whose discreteness is an immediate consequence of the semisimplicity of the category. (The semisimplicity of $*$-categories avoids appealing to the theorem of Barr-Beck in 31, 7] and to proceed in a pedestrian way, using only the definition of natural transformations.) The coinverse now arises from the conjugation in the category. The result is a discrete multiplier Hopf $*$-algebra. Thus our reconstruction of the discrete aqg is purely algebraic, the existence of the Haar functional following by quantum group theory.

The selfduality of the category of aqg and the existence of analytic extensions allow us to to relate our reconstruction result to those of Woronowicz and Yamagami on one hand and the purely algebraic ones on the other [34, 45. In particular, making use of the universal unitary corepresentation of an aqg introduced by $\mathrm{J}$. Kustermans [18], we prove that the tensor $*$-category of $*$-representations (or modules) of a discrete aqg $(A, \Delta)$ is equivalent to the tensor $*$-category of unitary corepresentations (or comodules) of the dual compact aqg $(\hat{A}, \hat{\Delta})$. We provide these categories with conjugates (for representations of discrete aqg this has not been worked out before). We also show that the tensor $*$-category of pointwise continuous finite dimensional $*$-representations of a sub Hopf $*$-algebra of the maximal dual (or Sweedler dual) Hopf $*$-algebra of a compact quantum group is equivalent to any of the tensor $*$-categories mentioned above, whenever the Hopf $*$-algebra separates the regular functions associated with the compact quantum group. This is a useful result since it applies to the deformed universal enveloping Lie algebras $U_{q}(\mathfrak{g})$ of M. Jimbo and V.G. Drinfeld. It therefore links these axiomatic quantum group results to the more familiar context of quantum groups given by deformations of semisimple Lie algebras $\mathfrak{g}$. Indeed, this is how the latter can be shown to produce compact (or discrete) quantum groups and how Woronowicz [1] constructed the compact matrix pseudogroup $S U_{q}(N)$. 
The correspondence between infinite dimensional representations and corepresentations was already established in [28] and [18], but only for the objects of the respective categories, morphisms and the tensor structure were not considered. In 39] tensor structure and braiding were taken into account in a purely algebraic context and, while studying the amenability of quantum groups, the correspondence between tensor $\mathrm{C}^{*}$-categories of infinite dimensional representations and corepresentations was established in [3, \#, for the various analytic versions of aqg and lcqg. The latter results rely on the theory of infinite dimensional representations and corepresentations and of the construction of the universal corepresentation for lcqg developed in [19]. But none of this work touched on Tannaka-Krein reconstruction, since conjugates do not exist in the infinite dimensional case. Another type of reconstruction result for tensor $\mathrm{C}^{*}$-categories involving infinite dimensional objects was undertaken in 10] from the point of view of multiplicative unitaries and the regular corepresentation.

For a discrete aqg $(A, \Delta)$ we establish a bijection between braidings of the category $\operatorname{Rep}_{f}(A, \Delta)$ and $R$ matrices in the multiplier algebra $M(A \otimes A)$. To the best of our knowledge this is the first such result rigorously proven in an axiomatic framework for quantum groups. If the category is symmetric and the embedding functor maps the braiding into the canonical braiding of the category of Hilbert spaces, the discrete aqg is cocommutative.

For any discrete aqg there is a compact group $G$, the intrinsic group, and for cocommutative $(A, \Delta)$ we prove an equivalence $\operatorname{Rep}_{f}(A, \Delta) \simeq \operatorname{Rep}_{f} G$ of tensor $*$-categories. This is the only point in our approach were analysis plays a role, in that we use the theorems of Gelfand, Krein-Milman, Stone-Weierstrass, etc. In the theory of locally compact quantum groups it is well known that commutative and cocommutative quantum groups are Kac algebras. Furthermore, commutative (resp. cocommutative) lcqg are of the form $(C(G), \Delta)$ (resp. $\left.\left(C_{r}^{*}(G), \Delta\right)\right)$ for a locally compact group $G$. Finally, a commutative compact aqg is the algebra of regular functions on a compact group $G$, but cocommutative discrete aqg are inconvenient to characterize. This is why, alternatively, we give the more instructive and direct proof of the above equivalence of categories. In passing we give a description of a cocommutative aqg in terms of the intrinsic group $G$.

It cannot be emphasized strongly enough that all Tannaka-Krein type results discussed so far depend on the tensor $*$-category being concrete, i.e. coming with a faithful tensor functor into the category of Hilbert spaces. There are applications in pure mathematics and in quantum field theory, where such a functor is not given a priori. For symmetric categories, it was first shown by S. Doplicher and J. E. Roberts [9] that such a functor always exists. An alternative approach in a more algebraic setting was given by P. Deligne [8]. The questions of existence and uniqueness of an embedding functor will be addressed anew in a sequel to this paper.

The above discussion did not follow the order of presentation. Let us therefore give a brief overview of the organization of this paper. In the next section we provide some preliminaries on tensor $*$-categories and aqg. Concerning the former we are quite brief, since much of this material is almost universally known. Concerning the latter we focus in particular on the discrete and compact cases and discuss the examples related to groups. In Section 3 we treat the representation and corepresentation theory of discrete and compact aqg, respectively, from a tensor $*$-category point of view. To this end, we recall the universal corepresentation due to Kustermans, and we discuss conjugates in these categories. The special case of cocommutative discrete aqg is considered in Section 4. Section 5 is the heart of this paper. There we construct a discrete quantum semigroup from a tensor *-category, deriving the coinverse from the conjugation and leading to a discrete aqg. The precise statement of the generalized Tannaka-Krein theorem for quantum groups is then made in Theorem 5.25. In the final Section 6 we establish the bijection between braidings and $R$-matrices. In the case of a symmetric tensor $*$-category with symmetric embedding functor we recover the classical Tannaka-Krein theorem for compact groups.

\section{Preliminaries}

\section{$2.1 \quad$ Tensor Categories}

For the definitions of categories, functors and natural transformations we refer, e.g., to [24]. In this subsection we briefly recall some of the less standard notions of category theory which will be needed here, others will be introduced as we proceed. We may occasionally say 'arrows' instead of 'morphisms' in order to avoid confusion with algebra homomorphisms. All categories which we consider are essentially small, i.e. equivalent to a small 
category. We mostly speak of 'tensor categories' rather than 'monoidal categories' but cannot avoid the adjective 'monoidal'. In view of the coherence theorems for (braided, symmetric) tensor categories we may assume all tensor categories to be strict, satisfying $X \otimes(Y \otimes Z)=(X \otimes Y) \otimes Z$ for all $X, Y, Z$. Following widespread use, we also consider the tensor categories of vector spaces as strict, appealing to the canonical isomorphisms to identify $X \otimes(Y \otimes Z)$ with $(X \otimes Y) \otimes Z$. We often write $X Y$ in the place of $X \otimes Y$. By $\mathcal{H}$ we mean the strictification of Hilb. (We also suppress all other canonical isomorphisms in Hilb, identifying $B(H \otimes K)=B(H) \otimes B(K)$.) Note, however, that one cannot assume all tensor functors to be strict without losing generality. Thus we need the following.

Definition 2.1 Let $\mathcal{C}, \mathcal{D}$ be (strict) tensor categories. A functor $F: \mathcal{C} \rightarrow \mathcal{D}$ is weakly monoidal if there exist morphisms $d_{X, Y}: F(X) \otimes F(Y) \rightarrow F(X \otimes Y)$ for all $X, Y \in \mathcal{C}$ and a morphism $e: \mathbf{1}_{\mathcal{D}} \rightarrow F\left(\mathbf{1}_{\mathcal{C}}\right)$ such that

1. The morphisms $d_{X, Y}$ are natural w.r.t. both arguments.

2. For all $X, Y, Z \in \mathcal{C}$ the following diagram commutes:

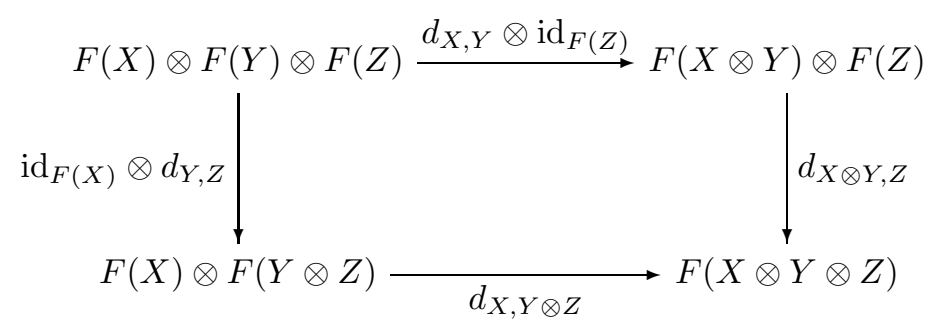

3. The following compositions are the identity morphisms of $F(X)$

$$
\begin{aligned}
& F(X) \equiv F(X) \otimes \mathbf{1}_{\mathcal{D}} \stackrel{\operatorname{id}_{F(X)} \otimes e}{\longrightarrow} F(X) \otimes F\left(\mathbf{1}_{\mathcal{C}}\right) \stackrel{d_{X, \mathbf{1}}}{\longrightarrow} F\left(X \otimes \mathbf{1}_{\mathcal{C}}\right) \equiv F(X) \\
& F(X) \equiv \mathbf{1}_{\mathcal{D}} \otimes F(X) \stackrel{e \otimes \operatorname{id}_{F(X)}}{\longrightarrow} F\left(\mathbf{1}_{\mathcal{C}}\right) \otimes F(X) \stackrel{d_{\mathbf{1}, X}}{\longrightarrow} F\left(\mathbf{1}_{\mathcal{C}} \otimes X\right) \equiv F(X)
\end{aligned}
$$

for all $X \in \mathcal{C}$.

The functor $F$ is monoidal if $e$ and all the $d_{X, Y}, X, Y \in \mathcal{C}$ are isomorphisms. If $\mathcal{C}, \mathcal{D}$ are tensor $*$-categories and $F$ is *-preserving, the isomorphisms $e, d_{X, Y}$ are required to be unitary.

We will mainly be interested in linear categories over the complex field. Viz., for all $X, Y, \operatorname{Mor}(X, Y)$ is a complex vector space, and the compositions $\circ, \otimes$ of morphisms are bilinear. All functors are supposed $\mathbb{C}$-linear. A $*$-operation on a $\mathbb{C}$-linear (tensor) category $\mathcal{C}$ is a map which assigns to an arrow $s \in \operatorname{Mor}(X, Y)$ another arrow $s^{*} \in \operatorname{Mor}(Y, X)$. This map has to be antilinear, involutive $\left(s^{* *}=s\right)$, contravariant $\left((s \circ t)^{*}=t^{*} \circ s^{*}\right)$ and, if $\mathcal{C}$ is monoidal, monoidal $\left((s \times t)^{*}=s^{*} \times t^{*}\right)$. A $*$-operation is positive iff $s^{*} \circ s=0$ implies $s=0$. A (tensor) $*$-category is a $\mathbb{C}$-linear (tensor) category with a positive $*$-operation. A morphism $s: X \rightarrow Y$ is an isometry iff it satisfies $s^{*} \circ s=\mathrm{id}_{X}$. A morphism $s$ is unitary iff $s$ and $s^{*}$ are isometries. A functor $F$ between *-categories is $*$-preserving if $F\left(s^{*}\right)=F(s)^{*}$ for every morphism $s$.

An object $Z$ is a direct sum of $X_{1}, X_{2}$, denoted $Z \cong X_{1} \oplus X_{2}$, if there are isometries $s_{i}: X_{i} \rightarrow Z, i=1,2$ such that $s_{1} \circ s_{1}^{*}+s_{2} \circ s_{2}^{*}=\mathrm{id} Z$. A $*$-category 'has direct sums' if for every pair $X_{1}, X_{2}$ there exists a direct sum. An object $Y$ is a subobject of $X$ if there exists an isometry $s: Y \rightarrow X$. A *-category 'has subobjects' if for every $p=p \circ p=p^{*} \in \operatorname{End}(X)$ there exist $Y$ and an isometry $s: Y \rightarrow X$ such that $s \circ s^{*}=p$. An object $X$ in a $\mathbb{C}$-linear category is irreducible if it is non-zero and End $X=\mathbb{C}$ id $X$.

Definition $2.2 A$ *-category is semisimple if it has finite dimensional spaces of morphisms, a zero object, direct sums and subobjects. A tensor *-category is semisimple if, in addition, the tensor unit $\mathbf{1}$ is irreducible. 
In a semisimple $*$-category $\mathcal{C}, \operatorname{End}(X)$ is a finite dimensional $C^{*}$-algebra for every $X$, and every object is a finite direct sum of irreducible objects. (It is well-known that a category which is semisimple in our sense is abelian and semisimple in the usual sense, i.e. all exact sequences split.)

Let $\mathcal{C}$ be a tensor $*$-category and $X \in \mathcal{C}$. A 'solution of the conjugate equations' is a triple $(\bar{X}, r, \bar{r})$, where $\bar{X} \in \mathcal{C}$ and $r: \mathbf{1} \rightarrow \bar{X} \otimes X, \bar{r}: \mathbf{1} \rightarrow X \otimes \bar{X}$ satisfy

$$
\begin{aligned}
& \bar{r}^{*} \otimes \operatorname{id}_{X} \circ \operatorname{id}_{X} \otimes r=\operatorname{id}_{X}, \\
& r^{*} \otimes \operatorname{id}_{\bar{X}} \circ \operatorname{id}_{\bar{X}} \otimes \bar{r}=\operatorname{id}_{\bar{X}} .
\end{aligned}
$$

A tensor $*$-category $\mathcal{C}$ 'has conjugates' if there is a solution of the conjugate equations for every $X \in \mathcal{C}$. A solution $(X, r, \bar{r})$ is normalized iff $r^{*} \circ r=\bar{r}^{*} \circ \bar{r}$. It is a standard solution iff there are irreducible objects $X_{i}, i \in I_{X}$, solutions $\left(\overline{X_{i}}, r_{i}, \bar{r}_{i}\right)$ of the conjugate equations and isometries $v_{i}: X_{i} \rightarrow X, w_{i}: \overline{X_{i}} \rightarrow \bar{X}, i \in I_{X}$ satisfying

$$
v_{i}^{*} \circ v_{j}=\delta_{i j} \operatorname{id}_{X_{i}}, \quad \sum_{i} v_{i} \circ v_{i}^{*}=\mathrm{id}_{X},
$$

similar equations for $w_{i}$, and we have

$$
r=\sum_{i} w_{i} \otimes v_{i} \circ r_{i}, \quad \bar{r}=\sum_{i} v_{i} \otimes w_{i} \circ \overline{r_{i}} .
$$

We define the (intrinsic or categorical) dimension $d(X) \in \mathbb{R}_{+}$of $X$ by $r^{*} \circ r=d(X) \operatorname{id}_{\mathbf{1}}$ where $(\bar{X}, r, \bar{r})$ is a normalized standard solution. One can prove the following facts, cf. 23]. The dimension is additive under direct sums and multiplicative under tensor products. It takes values in the set $\left\{2 \cos \frac{\pi}{n}, n=3,4, \ldots\right\} \cup[2, \infty)$, in particular $d(X) \geq 1$ with $d(X)=1$ iff $X \otimes \bar{X} \cong \mathbf{1}$ iff $X$ is invertible, i.e. there exists $Y$ such that $X \otimes Y \cong \mathbf{1}$. If $d(X)=1$ then $X$ is irreducible. In the category $\mathcal{H}$ of Hilbert spaces we have $d_{\mathcal{H}}(H)=\operatorname{dim}_{\mathbb{C}} H$.

We briefly comment on a somewhat more general setting. A $C^{*}$-(tensor) category is a (tensor) $*$-category where $\operatorname{Mor}(X, Y)$ is a Banach space for every pair $(X, Y)$ of objects and the norms satisfy $\left\|X^{*} \circ X\right\|=\|X\|^{2}$. A $W^{*}$-category is a $C^{*}$-category where every $\operatorname{Mor}(X, Y)$ is the dual of a Banach space for every pair $(X, Y)$. In a $C^{*}$-category with conjugates and irreducible tensor unit all spaces of morphisms are finite dimensional. This is useful in applications where this finite dimensionality is not known a priori, like in quantum field theory. Conversely, every $*$-category which is semisimple in our sense is a $W^{*}$-category, cf. [25]. In a $W^{*}$-category, every morphism $s: X \rightarrow Y$ has a polar decomposition $s=p u$, where $p$ is positive and $u$ a partial isometry. As a consequence, whenever $\operatorname{Mor}(X, Y)$ contains a split monic (or isomorphism), it also contains an isometry (respectively, unitary). This shows that most of the above definitions, e.g., of direct sums, are equivalent to the the usual ones as given, e.g., in [24].

If $\mathcal{C}$ is a semisimple tensor $*$-category we denote the set of isomorphism classes of irreducible objects by $I_{\mathcal{C}}$. Let $\left(X_{i}, i \in I_{\mathcal{C}}\right)$ be a complete set of irreducible objects and write $d_{i}=d\left(X_{i}\right)$. We have $0 \in I_{\mathcal{C}}$ such that $X_{0} \cong \mathbf{1}$. If $\mathcal{C}$ has conjugates, $I_{\mathcal{C}}$ comes with an involution $i \mapsto \bar{\imath}$ such that $X_{\bar{\imath}}$ is a conjugate of $X_{i}$. For $i, j, k \in I_{\mathcal{C}}$ we define $N_{i j}^{k}=\operatorname{dim} \operatorname{Mor}\left(X_{k}, X_{i} \otimes X_{j}\right)$. These numbers satisfy the following properties.

1. For every pair $(i, j)$ there are only finitely many $k \in I_{\mathcal{C}}$ such that $N_{i j}^{k} \neq 0$. We have $X_{i} \otimes X_{j} \cong$ $\bigoplus_{k \in I_{\mathcal{C}}} N_{i j}^{k} X_{k}$ and thus $d_{i} d_{j}=\sum_{k} N_{i j}^{k} d_{k}$.

2. $\sum_{l} N_{i j}^{l} N_{l k}^{m}=\sum_{n} N_{i n}^{m} N_{j k}^{n}$ for all $i, j, k, m \in I_{\mathcal{C}}$.

3. $N_{i j}^{k}=N_{k \bar{\jmath}}^{i}=N_{\bar{\imath} k}^{j}=N_{\bar{k} i}^{\bar{\jmath}}=N_{j \bar{k}}^{\bar{\imath}}=N_{\bar{\jmath}, \bar{\imath}}^{\bar{k}}$ for all $i, j, k \in I_{\mathcal{C}}$.

4. $N_{i j}^{0}=\delta_{i, \bar{j}}$.

5. If $\mathcal{C}$ is braided, cf. Section 6, then $N_{i j}^{k}=N_{j i}^{k}$ for all $i, j, k \in I_{\mathcal{C}}$. 


\subsection{Algebraic Quantum Groups}

In this subsection we briefly outline those aspects of the theory of aqg that will be needed in the sequel. For the details and proofs, see the original references [35, 37.

Every algebra will be a (not necessarily unital) associative algebra over the complex field $\mathbb{C}$. The identity map on a set $V$ will be denoted by $\iota$. If $V$ and $W$ are linear spaces, $V^{\prime}$ denotes the linear space of linear functionals on $V$ and $V \otimes W$ denotes the linear space tensor product of $V$ and $W$. The flip map $\sigma$ from $V \otimes W$ to $W \otimes V$ is the linear map sending $v \otimes w$ onto $w \otimes v$, for all $v \in V$ and $w \in W$. If $V$ and $W$ are Hilbert spaces, $V \otimes W$ denotes their Hilbert space tensor product; we denote by $B(V)$ and $B_{0}(V)$ the $\mathrm{C}^{*}$-algebras of bounded linear operators and compact operators on $V$, respectively. If $V$ and $W$ are algebras, $V \otimes W$ denotes their algebra tensor product. If $V$ and $W$ are $\mathrm{C}^{*}$-algebras, then $V \otimes W$ will denote their $\mathrm{C}^{*}$-tensor product with respect to the minimal $\mathrm{C}^{*}$-norm.

An algebra $A$ is non-degenerate if for any $a \in A$ such that $a b=0$ for all $b \in A$ or $b a=0$ for all $b \in A$, we have $a=0$. Obviously, all unital algebras are non-degenerate. If $A$ and $B$ are non-degenerate algebras, so is $A \otimes B$. From now on all algebras are assumed to be non-degenerate.

Let $A$ be a $*$-algebra and denote by End $A$ the unital algebra of linear maps from $A$ to itself. Let

$$
M(A)=\left\{x \in \text { End } A \mid \exists y \in \text { End } A \text { such that } x(a)^{*} b=a^{*} y(b) \quad \forall a, b \in A\right\} .
$$

Then $M(A)$ is a unital subalgebra of End $A$. The linear map $y$ associated to a given $x \in M(A)$ is uniquely determined by non-degeneracy and we denote it by $x^{*}$. The unital algebra $M(A)$ becomes a $*$-algebra when endowed with the involution $x \mapsto x^{*}$. This unital $*$-algebra is called the multiplier algebra of $A$.

Suppose that $A$ is an ideal in a $*$-algebra $B$. For $b \in B$, define $L_{b} \in M(A)$ by $L_{b}(a)=b a$, for all $a \in A$. Then the map $L: B \rightarrow M(A), b \mapsto L_{b}$, is a homomorphism. If $A$ is an essential ideal in $B$ in the sense that an element $b$ of $B$ is necessarily equal to zero if $b a=0$, for all $a \in A$, or $a b=0$, for all $a \in A$, then $L$ is injective. In particular, $A$ is an essential ideal in itself (by non-degeneracy) and therefore we have an injective homomorphism $L: A \rightarrow M(A)$. We identify the image of $A$ under $L$ with $A$. Then $A$ is an essential ideal of $M(A)$. (In fact, $M(A)$ is the 'largest' algebra containing $A$ as an essential ideal.) Obviously, $M(A)=A$ iff $A$ is unital.

If $A$ and $B$ are $*$-algebras, then it is easily verified that $A \otimes B$ is an essential self-adjoint ideal in $M(A) \otimes M(B)$. Hence, by the preceding remarks, there exists a canonical injective $*$-homomorphism from $M(A) \otimes M(B)$ into $M(A \otimes B)$. We use this to identify $M(A) \otimes M(B)$ as a unital $*$-subalgebra of $M(A \otimes B)$. In general, these algebras are not equal. A linear map $\pi: A \rightarrow B$ is said to be non-degenerate if $\pi(A) B=B$ and $B \pi(A)=B$. Here, as elsewhere, $\pi(A) B$ denotes the linear span of $\{\pi(a) b \mid a \in A, b \in B\}$. Whenever $\pi$ is non-degenerate and multiplicative (resp. non-degenerate and antimultiplicative), there exists a unique extension to a unital homomorphism (resp. antihomomorphism) $\bar{\pi}: M(A) \rightarrow M(B)$. We shall henceforth use the same symbol $\pi$ to denote the original map and its extension $\bar{\pi}$. A representation of a $*$-algebra is a non-degenerate homomorphism $\pi: A \rightarrow B(K)$, where $K$ is a Hilbert space.

If $\omega$ is a linear functional on $A$ and $x \in M(A)$, we define the linear functionals $x \omega$ and $\omega x$ on $A$ by setting $(x \omega)(a)=\omega(a x)$ and $(\omega x)(a)=\omega(x a)$, for all $a \in A$. We need the leg numbering notation. Take three $*$-algebras $A, B, C$. It can be shown that there exists a non-degenerate $*$-homomorphism $\theta_{13}: A \otimes C \rightarrow M(A \otimes B \otimes C)$ such that $\theta_{13}(a \otimes c)=a \otimes 1 \otimes c$, for all $a \in A, c \in C$. Thus, it has a unique extension to $M(A \otimes C)$. Set $x_{13}=\theta_{13}(x)$, for all $x \in M(A \otimes C)$. The other variants of the leg numbering notation are defined similarly.

The triple product $A \otimes A \otimes A$ is an essential ideal of both $M(A \otimes A) \otimes A$ and $A \otimes M(A \otimes A)$, thus $M(M(A \otimes A) \otimes A) \subset M(A \otimes A \otimes A)$ and $M(A \otimes M(A \otimes A)) \subset M(A \otimes A \otimes A)$.

Definition 2.3 A multiplier Hopf $*$-algebra $(A, \Delta)$ consists of $a *$-algebra $A$ and $a *$-homomorphism $\Delta$ from $A$ into $M(A \otimes A)$ such that

1. $(\Delta \otimes \iota) \Delta=(\iota \otimes \Delta) \Delta$.

2. The linear mappings $T_{1}, T_{2}$ from $A \otimes A$ into $M(A \otimes A)$ such that

$$
\begin{aligned}
& T_{1}(a \otimes b)=\Delta(a)(b \otimes 1), \\
& T_{2}(a \otimes b)=\Delta(a)(1 \otimes b)
\end{aligned}
$$


for all $a, b \in A$, are bijections from $A \otimes A$ to $A \otimes A$.

Here the (unique) extension of $\Delta \otimes \iota: A \otimes A \rightarrow M(A \otimes A \otimes A)$ to $M(A \otimes A)$ is understood and similarly for $\iota \otimes \Delta$. Condition (ii) implies that $\Delta(a)(b \otimes 1), \Delta(a)(1 \otimes b) \in M(A \otimes A)$ lie in fact in $A \otimes A$.

We say that two multiplier Hopf $*$-algebras $\left(A_{1}, \Delta_{1}\right)$ and $\left(A_{2}, \Delta_{2}\right)$ are isomorphic, and write $\left(A_{1}, \Delta_{1}\right) \cong$ $\left(A_{2}, \Delta_{2}\right)$, if there exists a bijective $*$-homomorphism $\theta: A_{1} \rightarrow A_{2}$ such that $(\theta \otimes \theta) \Delta_{1}=\Delta_{2} \theta$.

The following result shows that multiplier Hopf $*$-algebras share most properties with Hopf algebras. Let $m: A \otimes A \rightarrow A$ denote the linear extension of the multiplication map. Note that $m$ is a $*$-homomorphism iff $A$ is commutative.

Proposition 2.4 Let $(A, \Delta)$ be a multiplier Hopf $*$-algebra. There exist a unique $*$-homomorphism $\varepsilon: A \rightarrow \mathbb{C}$ and a unique invertible antimultiplicative $S \in$ End $A$ such that

1. $(\varepsilon \otimes \iota)(\Delta(a)(1 \otimes b))=a b$,

2. $(\iota \otimes \varepsilon)(\Delta(a)(b \otimes 1))=a b$,

3. $m(S \otimes \iota)(\Delta(a)(1 \otimes b))=\varepsilon(a) b$,

4. $m(\iota \otimes S)(\Delta(a)(b \otimes 1))=\varepsilon(a) b$

for all $a, b \in A$. We call $\varepsilon$ the counit and $S$ the coinverse. Conversely, if $(A \otimes 1) \Delta(A) \subset A \otimes A$ and $(A \otimes 1) \Delta(A) \subset$ $A \otimes A$ and there exist linear maps $\varepsilon: A \rightarrow \mathbb{C}$ and $S: A \rightarrow A$ satisfying properties 1-4 with $S$ invertible, then $(A, \Delta)$ is a multiplier Hopf $*$-algebra.

We denote $m_{\mathrm{op}}=m \sigma$ and $\Delta_{\mathrm{op}}=\sigma \Delta$ where $\sigma(a \otimes b)=b \otimes a$ is the flip $*$-automorphism of $A \otimes A$ and $M(A \otimes A)$. $A_{\mathrm{op}}$ denotes the vector space $A$ with multiplication $m_{\mathrm{op}}$. The following result follows easily from the uniqueness of the counit and coinverse.

Proposition 2.5 The antipode $S$ is invertible and

1. $S^{-1}(a)=S\left(a^{*}\right)^{*} \quad \forall a \in A$.

2. $(S \otimes S)(\Delta(a)(1 \otimes b))=(1 \otimes S(b)) \Delta_{o p} S(a) \quad \forall a, b \in A$.

3. $\varepsilon S=\varepsilon$.

4. $\left(A_{o p}, \Delta\right)$ and $\left(A, \Delta_{o p}\right)$ are multiplier Hopf $*$-algebras with counit $\varepsilon$ and coinverse $S^{-1}$.

Definition 2.6 A linear functional $\omega$ on a multiplier Hopf $*$-algebra $(A, \Delta)$ is called

1. positive iff $\omega\left(a^{*} a\right) \geq 0 \quad \forall a \in A$,

2. faithful iff it is positive and $\omega\left(a^{*} a\right)=0 \Rightarrow a=0 \quad \forall a \in A$,

3. left-invariant iff $(\iota \otimes \omega)(\Delta(a)(b \otimes 1))=\omega(a) b \quad \forall a, b \in A$,

4. right-invariant iff $(\omega \otimes \iota)((1 \otimes b) \Delta(a))=\omega(a) b \quad \forall a, b \in A$.

Thus $\omega$ is left-invariant iff $\omega S$ is right-invariant.

Definition 2.7 An aqg is a multiplier Hopf $*$-algebra $(A, \Delta)$ which admits a non-zero left-invariant positive linear functional $\varphi$.

THEOREM 2.8 Let $(A, \Delta)$ be an aqg with positive left-invariant functional $\varphi$. Then

1. $\varphi$ is faithful. 
2. If $\omega$ is a left-invariant functional, there exists $c \in \mathbb{C}$ such that $\omega=c \varphi$.

3. If $\omega$ is a right-invariant functional, there exists $c \in \mathbb{C}$ such that $\omega=c \varphi S$.

An immediate consequence is that the $*$-operation is positive, viz. $a^{*} a=0 \Rightarrow a=0$, which clearly is stronger than non-degeneracy. Furthermore, there exists a unique complex number $\mu$ such that $\varphi S^{2}=\mu \varphi$. It can be proved that $|\mu|=1$, but it has recently been discovered that $\mu \neq 1$ for the quantum group version of $a x+b$ 38]. Every aqg $(A, \Delta)$ has the property that to any $a \in A$, there exists $c \in A$ such that $a c=c a=a$. Two aqg are said to be isomorphic if they are isomorphic as multiplier Hopf $*$-algebras and we use the same notation $\cong$ to denote this.

Set $\psi=\varphi S$. Then $\psi$ is a non-zero right-invariant linear functional on $A$. However, in general, $\psi$ will not be positive. It is known that there exists a non-zero positive right-invariant linear functional on $A$. To $\varphi$ there existence a unique automorphism $\rho$ on $A$ such that $\varphi(a b)=\varphi(b \rho(a))$, for every $a, b \in A$. We refer to this as the weak KMS-property of $\varphi$. Moreover, we have $\Delta \rho=\left(S^{2} \otimes \rho\right) \Delta$ and $\rho\left(\rho\left(a^{*}\right)^{*}\right)=a$, for every $a \in A$. Also there exists an automorphism $\rho^{\prime}$ for the right-invariant functional $\psi$, that is, $\rho^{\prime}$ is an automorphism on $A$ such that $\psi(a b)=\psi\left(b \rho^{\prime}(a)\right)$, for every $a, b \in A$.

It is possible to introduce a modular function for aqg. It is an invertible element $\delta$ in $M(A)$ such that $(\varphi \otimes \iota)(\Delta(a)(1 \otimes b))=\varphi(a) \delta b$, for every $a, b \in A$. This modular function satisfies

$$
\Delta(\delta)=\delta \otimes \delta, \quad \varepsilon(\delta)=1, \quad S(\delta)=\delta^{-1} .
$$

As in the classical case we have

$$
\varphi(S(a))=\varphi(a \delta)=\mu \varphi(\delta a) \quad \forall a \in A .
$$

We now discuss duality within the category of aqg. Define a subspace $\hat{A}$ of $A^{\prime}$ by

$$
\hat{A}=\{\varphi a \mid a \in A\}=\{a \varphi \mid a \in A\} .
$$

Then $\hat{A}$ is a non-degenerate $*$-algebra under the definitions:

1. For every $\omega_{1}, \omega_{2} \in \hat{A}$ and $a \in A$, we have $\left(\omega_{1} \omega_{2}\right)(a)=\left(\omega_{1} \otimes \omega_{2}\right)(\Delta(a))$.

2. For every $\omega \in \hat{A}$ and $a \in A$, define $\omega^{*}(a)=\overline{\omega\left(S(a)^{*}\right)}$.

The comultiplication $\hat{\Delta}$ is defined on $\hat{A}$ by $\hat{\Delta}(\omega)(x \otimes y)=\omega(x y)$, for every $\omega \in \hat{A}$ and $x, y \in A$. For this to make sense, $M(A \otimes A)$ should be embedded in $(A \otimes A)^{\prime}$ in a proper way. A definition of the comultiplication $\hat{\Delta}$ that does not use such an embedding can be found in [18]. Then $(\hat{A}, \hat{\Delta})$ is a multiplier Hopf $*$-algebra with counit $\hat{\varepsilon}$ and the antipode $\hat{S}$ given by:

1. $\hat{\varepsilon}(\omega)=\omega(1)$, for every $\omega \in \hat{A}$.

2. $\hat{S}(\omega)(a)=\omega(S(a))$, for every $\omega \in \hat{A}$ and $a \in A$.

Define $\hat{a}=a \varphi \in \hat{A}$, for $a \in A$. The map $A \rightarrow \hat{A}$ sending $a$ to $\hat{a}$ is a bijection, and is referred to as the Fourier transform. Next define the linear functional $\hat{\psi}$ on $\hat{A}$ by setting $\hat{\psi}(\hat{a})=\varepsilon(a)$, for every $a \in A$. It is possible to prove that $\hat{\psi}$ is right-invariant, and that $\hat{\psi}\left(\hat{a}^{*} \hat{a}\right)=\varphi\left(a^{*} a\right)$, for every $a \in A$. It follows that $\hat{\psi}$ is a non-zero positive linear functional on $\hat{A}$, and that the dual $(\hat{A}, \hat{\Delta})$ is an aqg. Let $\hat{\rho}$ denote the automorphism of $\hat{A}$ such that $\hat{\psi}(\hat{a} \hat{b})=\hat{\psi}(\hat{b} \hat{\rho}(\hat{a}))$, for all $a, b \in A$. The aqg version of Pontryagin's duality theorem takes the following form:

THEOREm 2.9 Let $(A, \Delta)$ be an aqg. Then the double dual $(\hat{\hat{A}}, \hat{\hat{\Delta}})$ is an aqg isomorphic to $(A, \Delta)$. More precisely, there exists a canonical $*$-isomorphism $\theta: A \rightarrow \hat{\hat{A}}$ such that $\theta(a)(\omega)=\omega(a)$, for all $a \in \hat{A}$, and that satisfies the equation $(\theta \otimes \theta) \Delta=\hat{\hat{\Delta}} \theta$. 
Note that an aqg $(A, \Delta)$ is commutative iff $(\hat{A}, \hat{\Delta})$ is cocommutative.

We shall need to consider an object associated to an aqg called its analytic extension. See [20] for full details. If $A$ is a $\mathrm{C}^{*}$-algebra, then $M(A)$ denotes the usual multiplier algebra of $A$, and $*$-homomorphism between $\mathrm{C}^{*}$ -

algebras are assumed to be non-degenerate in the usual operator algebraic sense. We need first to recall the concept of a GNS pair. Suppose given a positive linear functional $\omega$ on a $*$-algebra $A$. Let $H$ be a Hilbert space, and let $\Lambda: A \rightarrow H$ be a linear map with dense range for which $(\Lambda(a), \Lambda(b))=\omega\left(b^{*} a\right)$, for all $a, b \in A$. Then we call $(H, \Lambda)$ a GNS pair associated to $\omega$. As is well known, such a pair always exists and is essentially unique.

If $\varphi$ is a left Haar integral on an aqg $(A, \Delta)$, and $(H, \Lambda)$ is an associated GNS pair, then it can be shown that there is a unique $*$-homomorphism $\pi: A \rightarrow B(H)$ such that $\pi(a) \Lambda(b)=\Lambda(a b)$, for all $a, b \in A$. Moreover, $\pi$ is faithful and non-degenerate. We let $A_{r}$ denote the norm closure of $\pi(A)$ in $B(H)$. Thus, $A_{r}$ is a non-degenerate $\mathrm{C}^{*}$-subalgebra of $B(H)$. There exists a unique non-degenerate $*$-homomorphism $\Delta_{\mathrm{r}}: A_{r} \rightarrow M\left(A_{r} \otimes A_{r}\right)$ such that, for all $a \in A$ and all $x \in A \otimes A$, we have $\Delta_{\mathrm{r}}(\pi(a))(\pi \otimes \pi)(x)=(\pi \otimes \pi)(\Delta(a) x)$ and $(\pi \otimes \pi)(x) \Delta_{\mathrm{r}}(\pi(a))=$ $(\pi \otimes \pi)(x \Delta(a))$.

Define a linear map $\hat{\Lambda}: \hat{A} \rightarrow H$ by setting $\hat{\Lambda}(\hat{a})=\Lambda(a)$, for all $a \in \mathcal{A}$. Since $\hat{\psi}\left(\hat{b}^{*} \hat{a}\right)=\varphi\left(b^{*} a\right)=(\Lambda(a), \Lambda(b))$, for all $a, b \in A$, it follows that $(H, \hat{\Lambda})$ is a GNS-pair associated to $\hat{\psi}$. It can be shown that it is unitarily equivalent to the GNS-pair for a left Haar integral $\hat{\varphi}$ of $(\hat{A}, \hat{\Delta})$. Hence, we can use $(H, \hat{\Lambda})$ to define a representation of the analytic extension $\left(\hat{A}_{r}, \hat{\Delta}_{\mathrm{r}}\right)$ of $(\hat{A}, \hat{\Delta})$ on the space $H$. There is a unique $*$-homomorphism $\hat{\pi}_{r}: \hat{A} \rightarrow B(H)$ such that $\hat{\pi}_{r}(a) \hat{\Lambda}(b)=\hat{\Lambda}(a b)$, for all $a, b \in \hat{A}$. Moreover, $\hat{\pi}$ is faithful and non-degenerate. Let $\hat{A}_{r}$ be the norm closure of $\hat{\pi}_{r}(A)$ in $B(H)$, so $\hat{A}_{r}$ is a non-degenerate $\mathrm{C}^{*}$-subalgebra of $B(H)$. There exist a unique $*$-homomorphism $\hat{\Delta}_{r}: \hat{A}_{r} \rightarrow M\left(\hat{A}_{r} \otimes \hat{A}_{r}\right)$ such that, for all $a \in \hat{A}$ and $x \in \hat{A} \otimes \hat{A}, \hat{\Delta}_{r}(\hat{\pi}(a))(\hat{\pi} \otimes \hat{\pi})(x)=(\hat{\pi} \otimes \hat{\pi})(\hat{\Delta}(a) x)$ and $(\hat{\pi} \otimes \hat{\pi})(x) \hat{\Delta}_{r}(\hat{\pi}(a))=(\hat{\pi} \otimes \hat{\pi})(x \hat{\Delta}(a))$.

\subsection{Discrete and Compact Case}

The following proposition is well known and easy to prove.

Proposition 2.10 Let $I$ be a set and $H_{i}, i \in I$ finite dimensional Hilbert spaces. Consider the algebraic direct sum

$$
A=\bigoplus_{i \in I} B\left(H_{i}\right)
$$

1. A is a $*$-algebra with the usual $*$-operation on $B\left(H_{i}\right)$.

2. A is unital iff I is finite.

3. The canonical embedding maps $\iota_{i}: B\left(H_{i}\right) \hookrightarrow A$ and projections $p_{i}: A \rightarrow B\left(H_{i}\right)$ are $*$-homomorphisms.

4. Let $M(A)$ be the multiplier algebra and $L: A \rightarrow M(A)$ the canonical embedding map. There exists a unique isomorphism

$$
P: M(A) \rightarrow \prod_{i \in I} B\left(H_{i}\right)
$$

such that $P L=\mathrm{id}$, where we use the obvious identification of the direct sum with a subalgebra of the direct product.

5. If $\pi$ is an irreducible (*)-representation of $A$ there is $i \in I$ and an invertible (unitary) $U \in B\left(H, H_{i}\right.$ ) unique up to a scalar such that

$$
\pi(\cdot)=\pi \iota_{i} p_{i}(\cdot)=U p_{i}(\cdot) U^{-1} .
$$

6. Any (*-)representation of $A$ is a direct sum of irreducible (*-)representations. 
From now on the isomorphism $P$ will be used to make an identification $M(A) \equiv \prod_{i \in I} B\left(H_{i}\right)$ and therefore suppressed.

Definition 2.11 A multiplier Hopf $*$-algebra $(A, \Delta)$ is called discrete if $A=\oplus_{i} B\left(H_{i}\right)$. An aqg is called discrete if it is discrete as a multiplier Hopf *-algebra.

The proof of the following result is included, since it is instructive for our purposes.

TheOREM 2.12 Let $(A, \Delta)$ be a discrete multiplier Hopf $*$-algebra. Then there is a unique $f \in M(A)$ satisfying

1. $f$ is positive and invertible,

2. $S^{2}(a)=f a f^{-1} \forall a \in A$,

3. $\operatorname{Tr}_{i} p_{i}(f)=\operatorname{Tr}_{i} p_{i}\left(f^{-1}\right)$, where $T r_{i}$ is the usual trace on $B\left(H_{i}\right)$.

It also satisfies $S(f)=f^{-1}$ (where the unique extension of $S$ to $M(A)$ is understood).

Proof. To simplify the formulae we introduce the antilinear map $\chi(a)=S\left(a^{*}\right)$ which satisfies $\chi^{2}=\operatorname{id}_{A}$. For every $i \in I$ we pick an antiunitary $J_{i}: H_{i} \rightarrow H_{i}$. Now, $J_{i} p_{i}(\cdot) J_{i}^{-1}$ is a (linear) irreducible representation of $A$ on $H_{i}$ for any $i \in I$. By Prop. 2.10 there is a unique $c(i) \in I$ and an invertible $V_{i}: H_{i} \rightarrow H_{c(i)}$ such that

$$
V_{i} J_{i} p_{i} \chi(\cdot) J_{i}^{-1} V_{i}^{-1}=p_{c(i)} .
$$

Now $U_{i} \equiv V_{i} J_{i}$ is unique up to a scalar. We calculate

$$
U_{i} p_{i}(a) U_{i}^{-1}=U_{i} p_{i}(\chi \chi(a)) U_{i}^{-1}=p_{c(i)}(\chi(a))=U_{c(i)}^{-1} p_{c(c(i))} U_{c(i)} .
$$

In the first step we have used $\chi^{2}=\mathrm{id}$ and then we have used (2.1) twice. This shows that the irreducible representations $p_{i}$ and $p_{c(c(i))}$ are equivalent and therefore $c$ is involutive: $c(c(i))=i$. From now on we write $\bar{\imath}=c(i)$. We have

$$
U_{\bar{\imath}} U_{i} p_{i}(\cdot) U_{i}^{-1} U_{\bar{\imath}}=p_{i}(\cdot),
$$

and by irreducibility $U_{\bar{\imath}} U_{i}=k_{i} 1 \in B\left(H_{i}\right)$. Using the freedom (up to a scalar) in the choice of the $U_{i}$ we can assume that $k_{i}=1 \forall i \in I$. (If $i=\bar{\imath}$ we can absorb $k_{i}$ in $U_{i}$. If $i \neq \bar{\imath}$, we can achieve $U_{\bar{\imath}} U_{i}=1$, and since the $U_{i}$ are invertible this implies that automatically $k_{\bar{\tau}}=1$.)

From (2.1) we obtain $p_{i} S(a)=U_{i}^{-1} p_{\bar{\imath}}\left(a^{*}\right) U_{i}$ and applying this twice yields

$$
p_{i} S^{2}(a)=U_{i}^{-1} p_{\bar{\imath}} S(a)^{*} U_{i}=U_{i}^{-1}\left[U_{\bar{\imath}}^{-1} p_{\overline{\bar{\imath}}}\left(a^{*}\right) U_{\bar{\imath}}\right]^{*} U_{i}=U_{i}^{-1} U_{\bar{\imath}}^{*} p_{i}(a) U_{\bar{\imath}}^{-1 *} U_{i} .
$$

Thus $p_{i} S^{2}(a)=G_{i} p_{i}(a) G_{i}^{-1}$ with $G_{i}=U_{i}^{-1} U_{\bar{\imath}}^{*}=k_{i}^{-1} U_{\bar{\imath}} U_{\bar{\imath}}^{*}$ positive and invertible for all $i \in I$. Defining

$$
F_{i}=\sqrt{\frac{\operatorname{Tr}_{i} G_{i}^{-1}}{\operatorname{Tr}_{i} G_{i}}} G_{i},
$$

we have

$$
\operatorname{Tr}_{i} F_{i}=\sqrt{\operatorname{Tr}_{i} G_{i}^{-1} \operatorname{Tr}_{i} G_{i}}=\operatorname{Tr}_{i} F_{i}^{-1}
$$

as required. Obviously, also the $F_{i}$ are positive and invertible and $p_{i} S^{2}(a)=F_{i} p_{i}(a) F_{i}^{-1}$. The uniqueness is obvious in view of the normalizations. It remains to prove $S(f)=f^{-1}$, which is clearly equivalent to $p_{i} S(f)=p_{i}\left(f^{-1}\right) \forall i$. As above, with (2.1) we have $p_{i} S(f)=U_{i}^{-1} p_{\bar{\imath}}\left(f^{*}\right) U_{i}=U_{i}^{-1} F_{\bar{\imath}}^{*} U_{i}$. On the other hand, $p_{i}\left(f^{-1}\right)=F_{i}^{-1}$. Thus we have to show

$$
U_{i}^{-1} F_{\bar{\imath}}^{*} U_{i}=F_{i}^{-1} \quad \forall i \in I
$$


Inserting the definition of the $F_{i}$ this is equivalent to

$$
U_{i}^{-1} \sqrt{\frac{T r_{\bar{\imath}} G_{\bar{\tau}}^{-1}}{T r_{\bar{\imath}} G_{\bar{\imath}}}} U_{i} U_{\bar{\imath}}^{-1 *} U_{i}=\sqrt{\frac{T r_{i} G_{i}}{T r_{i} G_{i}^{-1}}} U_{\bar{\imath}}^{*-1} U_{i} \quad \forall i \in I .
$$

This is clearly equivalent to

$$
\operatorname{Tr}_{\bar{\imath}} G_{\bar{\imath}}^{-1} \operatorname{Tr}_{i} G_{i}^{-1}=\operatorname{Tr}_{i} G_{i} \operatorname{Tr}_{\bar{\imath}} G_{\bar{\imath}} \quad \forall i \in I
$$

Plugging in $G_{i}=U_{i}^{-1} U_{\bar{\imath}}^{*}$ this is an easy computation which we omit. (One uses $U_{\bar{\imath}}=U_{i}^{-1}$ and cyclicity of the traces.)

TheOREM 2.13 A discrete multiplier Hopf $*$-algebra $(A, \Delta)$ admits a non-zero left-invariant positive linear functional $\varphi$, thus is an aqg.

Proposition 2.14 An aqg $(A, \Delta)$ is discrete iff there exists $h \in A$ such that ah $=\varepsilon(a) h$, for all $a \in A$.

Definition 2.15 An aqg $(A, \Delta)$ is called compact if $A$ is unital.

Note that an aqg $(A, \Delta)$ is compact iff it is a Hopf $*$-algebra. Hence the analytical extension of a compact aqg is a compact quantum group in the sense of S.L. Woronowicz. A multiplier Hopf $*$-algebra $(A, \Delta)$ with $A$ unital is not necessarily an aqg, but this is the case whenever $A$ has a $\mathrm{C}^{*}$-algebra envelope. It is easily seen that $(A, \Delta)$ is compact and discrete iff $A$ is finite dimensional.

TheOREM 2.16 Let $(A, \Delta)$ be an aqg. Then $(A, \Delta)$ is compact iff $(\hat{A}, \hat{\Delta})$ is discrete.

\subsection{Examples}

The examples discussed below are treated in depth in 111. The translation into the framework of aqgis straightforward and is recommended as an exercise.

Throughout this section let $\Gamma$ denote a discrete group with unit $e$. Consider the group algebra $\mathbb{C} \Gamma$ and the algebra $C_{c}(\Gamma)$ of finitely supported complex valued functions with the usual pointwise operations. They are both non-degenerate $*$-algebras. The group algebra has unit $\delta_{e}$, whereas $C_{c}(\Gamma)$ has a unit iff $\Gamma$ is finite.

In the group algebra case $(\mathbb{C} \Gamma, \Delta)$ the comultiplication is given by $\Delta\left(\delta_{g}\right)=\delta_{g} \otimes \delta_{g}$ and yields a Hopf $*$-algebra with counit $\varepsilon\left(\delta_{g}\right)=1$ and coinverse $S\left(\delta_{g}\right)=\delta_{g^{-1}}$, for all $g \in \Gamma$. The functional defined by $\varphi\left(\delta_{g}\right)=\delta_{g, e}$, for $g \in \Gamma$, is unital, positive and both left and right invariant, so $(\mathbb{C} \Gamma, \Delta)$ is a cocommutative compact aqg.

Note that $M\left(C_{c}(\Gamma)\right) \cong C(\Gamma)$, where $C(\Gamma)$ is the unital $*$-algebra of all functions on $\Gamma$, and that $C_{c}(\Gamma \times \Gamma) \cong$ $C_{c}(\Gamma) \otimes C_{c}(\Gamma)$ (as *-algebras). Thus the algebra $C_{c}(\Gamma)$ has comultiplication $\Delta: C_{c}(\Gamma) \rightarrow M\left(C_{c}(\Gamma) \otimes C_{c}(\Gamma)\right)$ given by $\Delta(a)(g, h)=a(g h)$ for $a \in C_{c}(\Gamma)$ and $g, h \in \Gamma$. It is easy to see that the support of $\Delta(a)$ for $a \in C_{c}(\Gamma)$ is infinite whenever $\Gamma$ is. This shows that the Hopf algebra framework is too restrictive to cover this example. For $\left(C_{c}(\Gamma), \Delta\right)$ we have $\varepsilon(a)=a(e)$ and $S(a)(g)=a\left(g^{-1}\right)$ where $a \in C_{c}(\Gamma \times \Gamma)$ and $g \in \Gamma$. The integral $\varphi(a)=\sum_{g \in \Gamma} a(g), a \in \mathbb{C} \Gamma$, w.r.t. the counting measure is positive and both left and right invariant, so $\left(C_{c}(\Gamma), \Delta\right)$ is a commutative discrete aqg.

The following proposition shows that these two examples exhaust the cocommutative compact aqg and the commutative discrete aqg.

Proposition 2.17 Let $(A, \Delta)$ be an aqg. Then

1. $(A, \Delta)$ is discrete and commutative iff there exists a discrete group $\Gamma$ such that $(A, \Delta) \cong\left(C_{c}(\Gamma), \Delta\right)$.

2. $(A, \Delta)$ is compact and co-commutative iff there exists a discrete group $\Gamma$ such that $(A, \Delta) \cong(\mathbb{C} \Gamma, \Delta)$. 
It is also easy to check that $(\hat{\mathbb{C}} \Gamma, \hat{\Delta}) \cong\left(C_{c}(\Gamma), \Delta\right)$ and $\left(C_{c} \hat{(}(\Gamma), \hat{\Delta}\right) \cong(\mathbb{C} \Gamma, \Delta)$, so they are dual to each other.

Giving a characterization of the commutative compact and the cocommutative discrete algebraic quantum groups on the algebraic level, is less immediate and requires some (co-) representation theory. For the moment we mention that the commutative-compact case can be described in terms of the algebra of regular functions on a compact group, and we make here an analytic statement concerning both cases.

Proposition 2.18 Let $(A, \Delta)$ be an aqg with analytic extension $\left(A_{r}, \Delta_{r}\right)$. Then

1. $(A, \Delta)$ is compact and commutative iff there exists a compact group $G$ such that $\left(A_{r}, \Delta_{r}\right) \cong\left(C(G), \Delta_{r}\right)$.

2. $(A, \Delta)$ is discrete and cocommutative iff there exists a compact group $G$ such that $\left(A_{r}, \Delta_{r}\right) \cong\left(C_{r}^{*}(G), \Delta_{r}\right)$.

Again these two cases are dual to each other. Here $C(G)$ denotes the $\mathrm{C}^{*}$-algebra of continuous functions on $G$ with uniform norm, whereas $C_{r}^{*}(G)$ denotes the reduced group $\mathrm{C}^{*}$-algebra of $G$. Since a compact group $G$ is amenable, $C_{r}^{*}(G)$ coincides with the universal group $\mathrm{C}^{*}$-algebra $C^{*}(G)$, and therefore, as we shall see very explicitly in the next section, the representation theory of a discrete cocommutative aqg coincides with that of $G$. We shall also give a simple algebraic description of the associated aqg in this case.

\section{Representation Theory}

\subsection{The Universal Corepresentation}

Throughout this section $(A, \Delta)$ stands for an arbitrary aqg and $B$ denotes a $*$-algebra. We recall the universal corepresentation $U$ of $(A, \Delta)$ and discuss its various properties [18.

Definition $3.1 A$ corepresentation of $(A, \Delta)$ on $B$ is an element $V \in M(A \otimes B)$ such that $(\Delta \otimes \iota) V=V_{13} V_{23}$.

Proposition 3.2 Suppose $V$ is a corepresentation of $(A, \Delta)$ on $B$. Then the following are equivalent:

1. $V$ is invertible in $M(A \otimes B)$ with $V^{-1}=(S \otimes \iota) V$.

2. $(\varepsilon \otimes \iota) V=1$.

3. $V(A \otimes B)=A \otimes B=(A \otimes B) V$.

A corepresentation $V$ satisfying these equivalent conditions is said to be non-degenerate.

Definition 3.3 A unitary corepresentation $V$ of $(A, \Delta)$ on $B$ is a corepresentation on $B$ which is a unitary element of $M(A \otimes B)$.

The following result is fundamental for corepresentations of aqg. We regard elements of $A \otimes \hat{A}$ as endomorphisms of $A$.

THEOREM 3.4 There exists a unique element $U \in M(A \otimes \hat{A})$ such that

$$
\begin{aligned}
& {[U(x \otimes \omega)](y)=(\iota \otimes \omega)(\Delta(y)(x \otimes 1))} \\
& {[(x \otimes \omega) U](y)=(\omega \otimes \iota)((1 \otimes x) \Delta(y))}
\end{aligned}
$$

for all $x, y \in A$ and $\omega \in \hat{A}$. Moreover, we have the following properties for $U$ :

1. $U$ is unitary in $M(A \otimes \hat{A})$.

2. $(\Delta \otimes \iota) U=U_{13} U_{23}$. 
3. $(\iota \otimes \hat{\Delta}) U=U_{12} U_{13}$.

4. $(\omega \otimes \iota) U=\omega$ for all $\omega \in \hat{A}$.

5. $(\iota \otimes a) U=a$ for all $a \in A$, where $a$ acts on $\hat{A}$ by the identification $A=\hat{\hat{A}}$.

Note that claims 1 and 2 say that $U$ is a unitary corepresentation of $(A, \Delta)$ on $\hat{A}$, whereas claims 1 and 3 say that $\sigma(U)$ is a unitary corepresentation of $(\hat{A}, \hat{\Delta})$ on $A$. Below we will explain why $U$ is called the universal corepresentation of $(A, \Delta)$. It follows that $\sigma(U)$ is the universal corepresentation of $(\hat{A}, \hat{\Delta})$.

Let $\operatorname{Hom}(A, B)$ denote the set of $*$-homomorphisms from $A$ to $M(B)$ satisfying $\theta(A) B=B$.

Proposition 3.5 Let $V \in M(A \otimes B)$ and consider the linear map

$$
\pi_{V}: \hat{A} \rightarrow M(B), \quad \omega \mapsto(\omega \otimes \iota) V .
$$

Then the following equivalences hold:

1. $V$ is a corepresentation (of $(A, \Delta)$ on $B$ ) iff $\pi_{V}$ is multiplicative.

2. $V$ is a non-degenerate corepresentation iff $\pi_{V}$ is multiplicative and non-degenerate.

3. $V$ is a unitary corepresentation iff $\pi_{V} \in \operatorname{Hom}(\hat{A}, B)$.

The universality of $U$ can now be formulated as follows.

Theorem 3.6 For any unitary corepresentation $V$ of $(A, \Delta)$ on $B$ and any $\theta \in \operatorname{Hom}(\hat{A}, B)$, we have:

1. $\left(\iota \otimes \pi_{V}\right) U=V$.

2. $\pi_{(\iota \otimes \theta) U}=\theta$.

The result remains valid for the non-degenerate situation without $*$-operation.

Definition 3.7 Let $B^{\prime}$ be non-degenerate $*$-algebra. For $\theta \in \operatorname{Hom}(A, B)$ and $\theta^{\prime} \in \operatorname{Hom}\left(A, B^{\prime}\right)$ we define $\theta \times \theta^{\prime} \in \operatorname{Hom}\left(A, B \otimes B^{\prime}\right)$ by $\theta \times \theta^{\prime}=\left(\theta \otimes \theta^{\prime}\right) \Delta$. If $V$ and $V^{\prime}$ are unitary corepresentations of $(A, \Delta)$ on $B$ and $B^{\prime}$, respectively, we define a unitary corepresentation $V \times V^{\prime}$ of $(A, \Delta)$ on $B \otimes B^{\prime}$ by $V \times V^{\prime}=V_{12} V_{13}^{\prime}$.

TheOREM 3.8 The map sending unitary corepresentations $V$ of $(A, \Delta)$ on $B$ to $\pi_{V} \in \operatorname{Hom}(\hat{A}, B)$, is a bijection. Let $V^{\prime}$ be a unitary corepresentation of $(A, \Delta)$ on $B^{\prime}$. Then $\pi_{V \times V^{\prime}}=\pi_{V} \times \pi_{V^{\prime}}$. For the universal corepresentation $U$ of $(A, \Delta)$ on $\hat{A}$ we have $\pi_{U}=\iota \in \operatorname{Hom}(\hat{A}, \hat{A})$, and finally for the trivial unitary corepresentation $1 \otimes 1 \in A \otimes B(\mathbb{C})$ of $(A, \Delta)$ we have $\pi_{1 \otimes 1}=\hat{\varepsilon}$, where $\hat{\varepsilon}$ is the counit of $(\hat{A}, \hat{\Delta})$.

Proof. The only part which is not immediate from the results above, is the identity $\pi_{V \times V^{\prime}}=\pi_{V} \times \pi_{V^{\prime}}$. To show this, first note that

$$
\begin{aligned}
V & \times V^{\prime}=V_{12} V_{13}^{\prime}=\left[\left(\iota \otimes \pi_{V}\right) U\right]_{12}\left[\left(\iota \otimes \pi_{V^{\prime}}\right) U\right]_{13}=\left(\iota \otimes \pi_{V} \otimes \pi_{V^{\prime}}\right)\left(U_{12} U_{13}\right) \\
& =\left(\iota \otimes \pi_{V} \otimes \pi_{V^{\prime}}\right)(\iota \otimes \hat{\Delta}) U=\left(\iota \otimes\left(\pi_{V} \otimes \pi_{V^{\prime}}\right) \hat{\Delta}\right) U=\left(\iota \otimes \pi_{V} \times \pi_{V^{\prime}}\right) U .
\end{aligned}
$$

Thus we may conclude that

$$
\pi_{V \times V^{\prime}}(\omega)=(\omega \otimes \iota \otimes \iota)\left(V \times V^{\prime}\right)=(\omega \otimes \iota \otimes \iota)\left(\iota \otimes \pi_{V} \times \pi_{V^{\prime}}\right) U=\left(\pi_{V} \times \pi_{V^{\prime}}\right)(\omega \otimes \iota) U=\left(\pi_{V} \times \pi_{V^{\prime}}\right)(\omega),
$$

for all $\omega \in \hat{A}$. 
REMARK 3.9 The correspondence established in Theorem 3.8 is in fact a functor if we define arrows for representations and corepresentations on $*$-algebras in the following way. For any objects $\pi \in \operatorname{Hom}(A, B)$ and $\pi^{\prime} \in \operatorname{Hom}\left(A, B^{\prime}\right)$ define an arrow $f: \pi \rightarrow \pi^{\prime}$ to be $f \in \operatorname{Hom}\left(B, B^{\prime}\right)$ such that $f \pi=\pi^{\prime}$. Similarly, if $V$ and $V^{\prime}$ are unitary corepresentations of $(A, \Delta)$ on $B$ and $B^{\prime}$, respectively, we define an arrow $f: V \rightarrow V^{\prime}$ between these objects to be $f \in \operatorname{Hom}\left(B, B^{\prime}\right)$ such that $V^{\prime}=(\iota \otimes f) V$. Then the correspondence $V \mapsto \pi_{V}$ in Theorem 3.8 is an equivalence of tensor categories (they are not $*$-categories).

REMARK 3.10 We remark on the tensor categories of actions and coactions of an aqg $(A, \Delta)$ on $*$-algebras.

By an action $\gamma$ of $(A, \Delta)$ on a $*$-algebra $B$, we mean a surjective linear map $\gamma: A \otimes B \rightarrow B$ such that $\gamma(m \otimes \iota)=\gamma(\iota \otimes \gamma)$. An arrow $f: \gamma \rightarrow \gamma^{\prime}$ is an element $f \in \operatorname{Hom}\left(B, B^{\prime}\right)$ such that $f \gamma=\gamma^{\prime} f$. We do not define tensor products in the general case.

Given $\pi \in \operatorname{Hom}(A, B)$, we define the action $\gamma_{\pi}$ of $(A, \Delta)$ on $B$ by $\gamma_{\pi}(a \otimes b)=\pi(a) b$, for all $a \in A$ and $b \in B$. Using non-degeneracy of $f$, we see that for any $f \in \operatorname{Hom}\left(B, B^{\prime}\right)$, we have $f: \pi \rightarrow \pi^{\prime}$ iff $f: \gamma_{\pi} \rightarrow \gamma_{\pi^{\prime}}$. Thus $\pi \mapsto \gamma_{\pi}$ is an equivalence of the tensor category of non-degenerate $*$-representations of $(A, \Delta)$ on $*$-algebras to the tensor category of actions of $(A, \Delta)$ on $*$-algebras contained in the image of the functor and with tensor product $\gamma_{\pi} \times \gamma_{\pi^{\prime}}$ simply defined to be the action $\gamma_{\pi \times \pi^{\prime}}$. We have not incorporated the $*$-preserving property of $\pi$ in $\gamma_{\pi}$ and the axioms for actions.

A coaction $\delta$ of $(A, \Delta)$ on a $*$-algebra $B$ is a $\delta \in \operatorname{Hom}(B, A \otimes B)$ such that $(\iota \otimes \delta) \delta=(\Delta \otimes \iota) \delta$ and $(\varepsilon \otimes \iota) \delta=\iota$. Such coactions form a tensor category, an arrow $f: \delta \rightarrow \delta^{\prime}$ being an element $f \in \operatorname{Hom}\left(B, B^{\prime}\right)$ such that $(\iota \otimes f) \delta=\delta^{\prime} f$ and the tensor product $\delta \times \delta^{\prime}$ being the comodule of $(A, \Delta)$ on $B \otimes B^{\prime}$ given by $\left(\delta \times \delta^{\prime}\right)\left(b \otimes b^{\prime}\right)=\delta(b)_{12} \delta^{\prime}\left(b^{\prime}\right)_{13}$, for all $b \in B$ and $b^{\prime} \in B^{\prime}$.

Let $V$ be a unitary corepresentation of $(A, \Delta)$ on $B$. Then the linear map $\delta_{V}: B \rightarrow M(A \otimes B)$ given by $\delta_{V}(b)=V(1 \otimes b) V^{*}$ for all $b \in B$, is indeed a coaction of $\left(A, \Delta^{o p}\right)$ on $B$. Non-degeneracy of $\delta_{V}$ follows from $V(A \otimes B)=A \otimes B$, and the formula $\left(\iota \otimes \delta_{V}\right) \delta_{V}=\left(\Delta^{o p} \otimes \iota\right) \delta_{V}$ follows from the calculation

$$
\begin{aligned}
& \left(\iota \otimes \delta_{V}\right) \delta_{V}(b)=\left(\iota \otimes \delta_{V}\right) V\left(1 \otimes \delta_{V}(b)\right)\left(\left(\iota \otimes \delta_{V}\right) V\right)^{*}=V_{23} V_{13} V_{23}^{*}\left(1 \otimes V(1 \otimes b) V^{*}\right)\left(V_{23} V_{13} V_{23}^{*}\right)^{*} \\
& \quad=V_{23} V_{13}(1 \otimes 1 \otimes b) V_{13}^{*} V_{23}^{*}=\left(\Delta^{o p} \otimes \iota\right) V(1 \otimes 1 \otimes b)\left(\left(\Delta^{o p} \otimes \iota\right) V\right)^{*}=\left(\Delta^{o p} \otimes \iota\right)\left(V(1 \otimes b) V^{*}\right) \\
& \quad=\left(\Delta^{o p} \otimes \iota\right) \delta_{V}(b)
\end{aligned}
$$

for all $b \in B$. Note that any arrow $f: V \rightarrow V^{\prime}$ will be an arrow $f: \delta_{V} \rightarrow \delta_{V^{\prime}}$, but the converse is not true (consider $B^{\prime}$ commutative). So we have a tensor functor $V \mapsto \delta_{V}$ from the tensor category of unitary corepresentations of $(A, \Delta)$ on $*$-algebras to the tensor category of coactions of $\left(A, \Delta^{o p}\right)$ on $*$-algebras, which in general, is not an equivalence.

\subsection{Representations vs. Corepresentations as Tensor $*$-Categories}

We now proceed to establish the correspondence between representations of an aqg $(A, \Delta)$ and corepresentations of $(\hat{A}, \hat{\Delta})$, restricting ourselves to finite dimensional Hilbert spaces. For homomorphisms $\pi: A \rightarrow \operatorname{End} K$ there are two different notions of non-degeneracy which, fortunately, are equivalent for $K$ finite dimensional.

Lemma 3.11 Let $A$ be an algebra and $K$ a finite dimensional vector space. For a homomorphism $\pi: A \rightarrow$ End $K$ the conditions $\pi(A)$ End $K=$ End $K$ and $\pi(A) K=K$ are equivalent.

Proof. For $K$ finite dimensional we clearly have $(\operatorname{End} K) K=K$. Assuming $\pi(A) \operatorname{End} K=\operatorname{End} K$ we compute

$$
\pi(A) K=\pi(A)((\operatorname{End} K) K)=(\pi(A) \operatorname{End} K) K=(\operatorname{End} K) K=K .
$$

Conversely, assume $\pi(A) K=K$. With the isomorphism $\alpha: K \otimes K^{*} \rightarrow$ End $K$ determined by $\alpha(x \otimes \phi)(z)=$ $x \phi(z)$ for $x, z \in K, \phi \in K^{*}$ we have $(s \alpha(x \otimes \phi))(z)=s x \phi(z)=\alpha(s x \otimes \phi)(z)$ and therefore

$$
\pi(A) \text { End } K=\pi(A) \alpha\left(K \otimes K^{*}\right)=\alpha\left((\pi(A) K) \otimes K^{*}\right)=\alpha\left(K \otimes K^{*}\right)=\text { End } K,
$$

as desired. 
Definition 3.12 Let $(A, \Delta)$ be an aqg. Let $\operatorname{Rep}_{f}(A, \Delta)$ denote the class of (algebraically) non-degenerate *-representations of $A$ on finite dimensional Hilbert spaces including the zero representation 0 . Let $\pi_{1}, \pi_{2} \in$ $\operatorname{Rep}_{f}(A, \Delta)$. Then $\pi_{i} \in \operatorname{Hom}\left(A, B\left(K_{i}\right)\right)$, so we define $\pi_{1} \times \pi_{2} \in \operatorname{Hom}\left(A, B\left(K_{1}\right) \otimes B\left(K_{2}\right)\right)=\operatorname{Hom}\left(A, B\left(K_{1} \otimes\right.\right.$ $\left.\left.K_{2}\right)\right) \subset \operatorname{Rep}_{f}(A, \Delta)$ according to Definition 3.7.

We regard $\operatorname{Rep}_{f}(A, \Delta)$ as a tensor $*$-category with representations as objects and intertwiners as arrows. (Recall that a zero representation 0 is not regarded as an irreducible object.) The tensor product is the one given in the definition above. Clearly, $\operatorname{Rep}_{f}(A, \Delta)$ is a tensor $*$-category with the counit $\varepsilon$ as the irreducible unit. Of course, the $*$-operation in the category is the usual Hilbert space adjoint of operators.

Definition 3.13 We say $V$ is a finite dimensional unitary corepresentation of an aqg $(A, \Delta)$ on a Hilbert space $K$ if $K$ is finite dimensional and $V$ is a unitary corepresentation of $(A, \Delta)$ on the unital *-algebra $B(K)$ of linear operators on $K$. Let $\operatorname{Corep}_{f}(A, \Delta)$ denote the class of all such unitary corepresentations including the zero corepresentation 0 . Let $V, V^{\prime} \in \operatorname{Corep}_{f}(A, \Delta)$. Then we define the unitary corepresentation $V \times V^{\prime}$ of $(A, \Delta)$ on $B(K) \otimes B\left(K^{\prime}\right)=B\left(K \otimes K^{\prime}\right)$ according to Definition 3.X. Clearly, we have $V \times V^{\prime} \in \operatorname{Corep}_{f}(A, \Delta)$.

We regard $\operatorname{Corep}_{f}(A, \Delta)$ as a tensor $*$-category with corepresentations as objects and intertwiners as arrows. Recall that $T \in B\left(K, K^{\prime}\right)$ is an intertwiner between two corepresentations $V, V^{\prime} \in \operatorname{Corep}_{f}(A, \Delta)$ iff $T(\omega \otimes \iota) V=$ $(\omega \otimes \iota) V^{\prime} T$, for all $\omega \in \hat{A}$. The tensor product is the one given in the definition above. Clearly, $\operatorname{Corep}_{f}(A, \Delta)$ is a tensor $*$-category with the unit $1 \otimes 1$ as the irreducible unit. Again, the $*$-operation in the category is the usual Hilbert space adjoint of operators.

The two tensor categories are related in the following way.

THEOREM 3.14 Let notation be as above. The correspondence

$$
P: \operatorname{Corep}_{f}(A, \Delta) \rightarrow \operatorname{Rep}_{f}(\hat{A}, \hat{\Delta}), \quad V \mapsto \pi_{V}
$$

provided by Proposition 3.5 together with the identity map on morphisms gives rise to an isomorphism of tensor *-categories.

Proof. The proof is immediate from Theorem 3.8.

REMARK 3.15 A similar result can be obtained for the infinite dimensional case. One needs then to talk about *-representations which are non-degenerate in the $C^{*}$-algebra sense, so $\pi \in \operatorname{Rep}(A, \Delta)$ iff the exists a (possibly infinite dimensional) Hilbert space $K$ and a $*$-homomorphism $\pi: A \rightarrow B(K)$ such that the vector space $\pi(A) K$ is dense in $K$. The tensor $*$-category $\operatorname{Corep}\left(A_{r}, \Delta_{r}\right)$ of unitary corepresentations on Hilbert spaces consists of Hilbert spaces $K$ and unitaries $V \in M\left(A_{r} \otimes B_{0}(K)\right)$ such that $\left(\Delta_{r} \otimes \iota\right) V=V_{13} V_{23}$. For the exact correspondence thus established, see [5].

We will look at another way of obtaining a tensor $*$-category $\operatorname{Rep}_{f}\left(A_{s}^{o}, \hat{\Delta}\right)$ from a compact aqg $(A, \Delta)$ which is equivalent to $\operatorname{Corep}_{f}(A, \Delta)$. This can sometimes come in very handy, especially when dealing with representations of quantized universal enveloping Lie algebras $U_{q}(\mathfrak{g})$.

First recall that $(A, \Delta)$ is a Hopf *-algebra with counit $\varepsilon$, coinverse $S$ and unit $I$, so the vector space $A^{\prime}$ of all linear functionals on $A$ is a unital *-algebra with unit $\varepsilon$, product $\omega \eta=(\omega \otimes \eta) \Delta \in A^{\prime}$ and $*$-operation $\omega^{*} \in A^{\prime}$ given by $\omega^{*}(a)=\overline{\omega\left(S(a)^{*}\right)}$, for all $a \in A$. Define a linear map $\hat{\Delta}: A^{\prime} \rightarrow(A \otimes A)^{\prime}$ by $\hat{\Delta}(\omega)(a \otimes b)=\omega(a b)$, for all $\omega \in A^{\prime}$ and $a, b \in A$. Consider the subspace $A^{o}$ of $A^{\prime}$ given by

$$
A^{o}=\left\{\omega \in A^{\prime} \mid \hat{\Delta}(\omega) \in A^{\prime} \otimes A^{\prime}\right\},
$$

where we understand the embedding $A^{\prime} \otimes A^{\prime} \subset(A \otimes A)^{\prime}$. Then $\left(A^{o}, \hat{\Delta}\right)$ is called the maximal dual Hopf $*$-algebra of $(A, \Delta)$. Note that for $\hat{f} \in M(\hat{A})$ as in Theorem 2.12, we have $\hat{f} \in A^{o}$ and $\hat{\Delta}(\hat{f})=\hat{f} \otimes \hat{f}$. Regard $A^{o}$ as a locally convex topological vector space with pointwise convergence, so $\omega_{\lambda} \rightarrow \omega$ in $A^{o}$ if $\omega_{\lambda}(a) \rightarrow \omega(a)$, for all $a \in A$. The continuous unital $*$-representations of $\left(A^{o}, \hat{\Delta}\right)$ on finite dimensional Hilbert spaces clearly form a tensor $*$-category with conjugates given by the same formulas as for $\operatorname{Rep}_{f}(\hat{A}, \hat{\Delta})$. Note that in general, the maximal dual Hopf $*$-algebra can be very small! 
Theorem 3.16 Let $(A, \Delta)$ be a compact aqg and let $\left(A_{s}^{o}, \hat{\Delta}\right)$ be a sub Hopf $*$-algebra of the maximal dual Hopf *-algebra of $(A, \Delta)$ which separates the elements of $A$. (Thus $A_{s}^{o}$ is a unital *-subalgebra of $A^{\circ}$ and $\hat{\Delta}$ is given by restriction). Consider the tensor $*$-category $\operatorname{Rep}_{f}\left(A_{s}^{o}, \hat{\Delta}\right)$ of continuous unital $*$-representations of $\left(A_{s}^{o}, \hat{\Delta}\right)$ on finite dimensional Hilbert spaces with arrows, *-operation and tensor product defined in the obvious way. Then the assignment

$$
N: \operatorname{Corep}_{f}(A, \Delta) \rightarrow \operatorname{Rep}_{f}\left(A_{s}^{o}, \hat{\Delta}\right), \quad V \mapsto \pi_{V} \text { with } \pi_{V}(\omega)=(\omega \otimes \iota) V, \forall \omega \in A_{s}^{o},
$$

together with the identity map on arrows, is an equivalence of tensor $*$-categories.

Proof. The only part which requires a proof is that any $\pi \in \operatorname{Rep}_{f}\left(A_{s}^{o}, \hat{\Delta}\right)$ is of the form $\pi_{V_{\pi}}$ for some $V_{\pi} \in$ $\operatorname{Corep}_{f}(A, \Delta)$. Thus let $\pi: A_{s}^{o} \rightarrow B(K)$ be a continuous unital $*$-representation of $A_{s}^{o}$ on a finite dimensional Hilbert space $K$. We will provide a $V_{\pi} \in A \otimes B(K)$ such that $\pi_{V_{\pi}}=\pi$. Using the fact that $A_{s}^{o}$ separates the elements of $A$, it is then clear that $V_{\pi}$ will be unitary, that $(\Delta \otimes \iota) V_{\pi}=\left(V_{\pi}\right)_{13}\left(V_{\pi}\right)_{23}$ and that the $*$-preserving tensor functor $N$ is an equivalence.

Now pick an orthonormal basis $\left(e_{i}\right)$ for $K$ and consider the system $m_{i j}$ of matrix units for $B(K)$ defined as $m_{i j}\left(e_{k}\right)=\delta_{j k} e_{i}$, for all $i, j, k$. Consider, for fixed $i$ and $j$, the linear functional on $A_{s}^{o}$ given by $\omega \mapsto\left(\pi(\omega) e_{j}, e_{i}\right)$ for all $\omega \in A_{s}^{o}$. Since $\pi$ is continuous w.r.t pointwise topology on $A_{s}^{o}$, it follows from [27, Proposition 2.4.4], that there exists a unique $a_{i j} \in A$ such that $\omega\left(a_{i j}\right)=\left(\pi(\omega) e_{j}, e_{i}\right)$, for all $\omega \in A_{s}^{o}$. Define now $V_{\pi} \in A \otimes B(K)$ by $V_{\pi}=\sum_{i j} a_{i j} \otimes m_{i j}$. By construction $\pi_{V_{\pi}}=\pi$ and we are done.

REMARK 3.17 It is known that for the usual quantized compact aqg $\left(A_{q}, \Delta\right)$, the associated quantized universal enveloping Lie algebras $U_{q}(\mathfrak{g})$ are Hopf $*$-algebras with $A_{s}^{o}=U_{q}(\mathfrak{g})$ which separate the elements of $A_{q}$ [17, Sect. 7.1.5]. Thus the tensor $*$-category of continuous unital $*$-representations of $\left(U_{q}(\mathfrak{g}), \hat{\Delta}\right)$ on finite dimensional Hilbert spaces is equivalent to $\operatorname{Corep}_{f}\left(A_{q}, \Delta\right)$. Also $\hat{f} \in U_{q}(\mathfrak{g})$, so the intrinsic dimension can be read off conveniently for such representations [30]. We return to this issue in the next subsection.

We now recast our results in the language of modules and comodules.

Proposition 3.18 Any non-degenerate $*$-representation $\pi$ of a discrete aqg $(A, \Delta)$ on a finite dimensional Hilbert space $K$ gives rise to an $A$-module $K$. Namely, define a linear map $\alpha_{\pi}: A \otimes K \rightarrow K$ by $\alpha_{\pi}(a \otimes \xi)=\pi(a) \xi$, for all $a \in A$ and $\xi \in K$. Then the following properties hold:

1. $\alpha_{\pi}(m \otimes \iota)=\alpha_{\pi}\left(\iota \otimes \alpha_{\pi}\right)$.

2. $\alpha_{\pi}(A \otimes K)=K$.

3. $\left(\alpha_{\pi}\left(a^{*} \otimes \xi\right), \eta\right)=\left(\xi, \alpha_{\pi}(a \otimes \eta)\right) \quad \forall a \in A \quad \forall \xi, \eta \in K$.

Denote by $\operatorname{Mod}_{f}(A, \Delta)$ the tensor $*$-category of linear maps $\alpha: A \otimes K \rightarrow K$ satisfying properties 1-3 above, with arrows $t: \alpha \rightarrow \alpha^{\prime}$ being linear maps $t: K \rightarrow K^{\prime}$ satisfying $\alpha^{\prime}=t \alpha$ and tensor product $\alpha \times \alpha^{\prime}: A \otimes K \otimes K^{\prime} \rightarrow$ $K \otimes K^{\prime}$ uniquely determined by

$$
\left(\alpha \times \alpha^{\prime}\right)(\iota \otimes \alpha \otimes \alpha)\left(a \otimes b \otimes \xi \otimes c \otimes \xi^{\prime}\right)=\sum_{k} \alpha\left(a_{k} \otimes \xi\right) \otimes \alpha^{\prime}\left(b_{k} \otimes \xi^{\prime}\right),
$$

for all $a, b \in A, \xi \in K$ and $\xi^{\prime} \in K^{\prime}$ and where $\sum_{k} a_{k} \otimes b_{k}=\Delta(a)(b \otimes c)$. Then the assignment $\pi \mapsto \alpha_{\pi}$ is a monoidal *-preserving equivalence of $\operatorname{Rep}_{f}(A, \Delta)$ and $\operatorname{Mod}_{f}(A, \Delta)$.

Proof. Straightforward, once one shows that $\alpha \times \alpha^{\prime}$ is well-defined. This is most easily done by considering the corresponding well-defined tensor product $\pi \times \pi^{\prime}$ of the associated non-degenerate $*$-representations, i.e, of $\pi$ and $\pi^{\prime}$ such that $\alpha=\alpha_{\pi}$ and $\alpha^{\prime}=\alpha_{\pi^{\prime}}$, and then noting that $\alpha \times \alpha^{\prime}=\alpha_{\pi \times \pi^{\prime}}$. 
Proposition 3.19 Any unitary corepresentation $V$ of a compact aqg $(A, \Delta)$ on a finite dimensional Hilbert space $K$ gives rise to an $A$-comodule $K$. Namely, define a linear map $\beta_{V}: K \rightarrow A \otimes K$ by $\beta_{V}(\xi)=\sum_{k} a_{k} \otimes x_{k} \xi$, for all $\xi \in K$ and where $V=\sum_{k} a_{k} \otimes x_{k} \in A \otimes B(K)$. Then the following properties hold:

1. $\left(\Delta^{o p} \otimes \iota\right) \beta_{V}=\left(\iota \otimes \beta_{V}\right) \beta_{V}$.

2. $(S \otimes(\cdot, \eta)) \beta_{V}(\xi)=(* \otimes(\xi, \cdot)) \beta_{V}(\eta) \quad \forall \xi, \eta \in K$,

where $\varepsilon$ and $S$ are the counit and coinverse of $(A, \Delta)$, respectively. Let $\operatorname{Comod}_{f}\left(A, \Delta^{o p}\right)$ denote the tensor $*$-category of linear maps $\beta: K \rightarrow A \otimes K$ satisfying properties 1-2 above, with arrows $t: \beta \rightarrow \beta^{\prime}$ being linear maps $t: K \rightarrow K^{\prime}$ satisfying $\beta^{\prime} t=(\iota \otimes t) \beta$ and tensor product $\beta \times \beta^{\prime}: K \otimes K^{\prime} \rightarrow A \otimes K \otimes K^{\prime}$ given by

$$
\left(\beta \times \beta^{\prime}\right)\left(\xi \otimes \xi^{\prime}\right)=\beta(\xi)_{12} \beta^{\prime}\left(\xi^{\prime}\right)_{13},
$$

for all $\xi \in K$ and $\xi^{\prime} \in K^{\prime}$. Then the assignment $V \mapsto \beta_{V}$ is a monoidal $*$-preserving equivalence of $\operatorname{Corep}_{f}(A, \Delta)$ and $\operatorname{Comod}_{f}\left(A, \Delta^{o p}\right)$.

Proof. Straightforward. How to produce a unitary corepresentation $V \in A \otimes B(K)$ of $(A, \Delta)$ from a prescribed comodule $\beta: K \rightarrow A \otimes K$ of $\left(A, \Delta^{o p}\right)$ perhaps calls for some explanation. Pick an orthonormal basis $\left(e_{k}\right)$ for $K$ and consider the system $\left(m_{i j}\right)$ of matrix units of $B(K)$ given by $m_{i j}\left(e_{k}\right)=\delta_{j k} e_{i}$ for all $i, j, k$. Define $V \in A \otimes B(K)$ by $V=\sum_{i j} a_{i j} \otimes m_{i j}$, where the elements $a_{i j} \in A$ are given by

$$
a_{i j}=\left(\iota \otimes\left(\cdot, e_{i}\right)\right) \beta\left(e_{j}\right),
$$

for all $i, j$. It is now easily checked that $\beta_{V}=\beta$.

We thus get the following restatement of Theorem 3.14 .

TheOREm 3.20 Let $(A, \Delta)$ be a discrete aqg. Then $\operatorname{Mod}_{f}(A, \Delta)$ and $\operatorname{Comod}_{f}\left(\hat{A}, \hat{\Delta}^{o p}\right)$ are equivalent as semisimple tensor $*$-categories.

\subsection{Conjugates for Representations and Corepresentations}

Suppose that $(A, \Delta)$ is a discrete aqg with counit $\varepsilon$ and coinverse $S$. We now show that the semisimple tensor *-categories $\operatorname{Rep}_{f}(A, \Delta)$ and $\operatorname{Corep}_{f}(\hat{A}, \hat{\Delta})$ have conjugates, and provide a conjugate object for every non-zero object.

Suppose $\pi$ is a non-zero non-degenerate $*$-representation on the finite dimensional Hilbert space $K$. Let $f \in M(A)$ be as in Theorem 2.12 and consider the positive operator $\pi\left(f^{-1}\right) \in B(K)$. Pick any Hilbert space $\bar{K}$ and any invertible antilinear operator $J: K \rightarrow \bar{K}$ such that $J^{*} J=\pi\left(f^{-1}\right)$. This can clearly be done. Now pick an orthonormal basis $\left(e_{i}\right)$ for $K$ and define linear maps $r: \mathbb{C} \rightarrow \bar{K} \otimes K$ and $\bar{r}: \mathbb{C} \rightarrow K \otimes \bar{K}$ by

$$
r(1)=\sum_{i} J e_{i} \otimes e_{i} \quad \text { and } \quad \bar{r}(1)=\sum_{i} e_{i} \otimes J^{*-1} e_{i},
$$

respectively. Next define

$$
\bar{\pi}: A \rightarrow B(\bar{K}), \quad a \mapsto J \pi S\left(a^{*}\right) J^{-1} .
$$

As $J$ and $J^{-1}$ both are antilinear, $\bar{\pi}(a)$ is linear and thus $\bar{\pi}(a) \in B(\bar{K})$ for all $a \in A$. By the following proposition, $\bar{\pi}$ is indeed a conjugate to $\pi$ and $r$ and $\bar{r}$ are normalized solutions of the conjugate equations for $\pi$ and $\bar{\pi}$.

Proposition 3.21 Let $(A, \Delta)$ be a discrete aqg and $\pi$ a non-zero object of $\operatorname{Rep}_{f}(A, \Delta)$. Then $\bar{\pi}$ is a non-zero object of $\operatorname{Rep}_{f}(A, \Delta)$. Moreover, the following hold:

1. $r \in \operatorname{Hom}(\varepsilon, \bar{\pi} \times \pi)$, 
2. $\bar{r} \in \operatorname{Hom}(\varepsilon, \pi \times \bar{\pi})$,

3. $\bar{r}^{*} \otimes \mathrm{id}_{\pi} \circ \mathrm{id}_{\pi} \otimes r=\mathrm{id}_{\pi}$,

4. $r^{*} \otimes \mathrm{id}_{\bar{\pi}} \circ \mathrm{id}_{\bar{\pi}} \otimes \bar{r}=\mathrm{id}_{\bar{\pi}}$,

5. $r^{*} \circ r=\bar{r}^{*} \circ \bar{r}$.

Proof. Claims 3 and 4 hold for any invertible antilinear map $J$, as is easily verified. Claim 5 is simply a restatement of the fact $\operatorname{Tr} \pi(f)=\operatorname{Tr} \pi\left(f^{-1}\right)$ stated in Theorem 2.12. To show that $\bar{\pi} \in \operatorname{Rep}_{f}(A, \Delta)$, we first note that $\bar{\pi}$ is linear (as $A d J$ and $*$ are both antilinear), multiplicative and non-degenerate, thus non-zero. To see that $\bar{\pi}$ is $*$-preserving, first notice that $S\left(a^{*}\right)^{*}=S^{-1}(a)$ and $S^{2}(a)=f a f^{-1}$ for all $a \in A$, and then calculate

$$
\bar{\pi}(a)^{*}=J^{*-1} \pi S^{-1}(a) J^{*}=J \pi(f) \pi S^{-1}(a) \pi\left(f^{-1}\right) J^{-1}=J \pi S(a) J^{-1}=\bar{\pi}\left(a^{*}\right),
$$

for all $a \in A$.

It remains to show relations 1 and 2. We prove only the first, the second being proved similarly. Now $r \in \operatorname{Hom}(\varepsilon, \bar{\pi} \times \pi)$ simply means that $\varepsilon(a) r(1)=(\bar{\pi} \times \pi)(a) r(1)$, for all $a \in A$. By the non-degeneracy of $\pi$ it thus suffices to show that

$$
\left(\varepsilon(a) r(1), J^{*-1} e_{j} \otimes \pi\left(b^{*}\right) e_{l}\right)=\left((\bar{\pi} \times \pi)(a) r(1), J^{*-1} e_{j} \otimes \pi\left(b^{*}\right) e_{l}\right)
$$

for all $a, b \in A$ and all $j, l$. On the l.h.s. we have

$$
\begin{aligned}
& \left(\varepsilon(a) r(1), J^{*-1} e_{j} \otimes \pi\left(b^{*}\right) e_{l}\right)=\varepsilon(a) \sum_{i}\left(J e_{i}, J^{*-1} e_{j}\right)\left(e_{i}, \pi\left(b^{*}\right) e_{l}\right) \\
& =\varepsilon(a) \sum_{i} \delta_{i j}\left(e_{i}, \pi\left(b^{*}\right) e_{l}\right)=\varepsilon(a)\left(e_{j}, \pi\left(b^{*}\right) e_{l}\right)=\left(\pi(\varepsilon(a) b) e_{j}, e_{l}\right) .
\end{aligned}
$$

To see that the r.h.s. coincides with this expression, first write $(1 \otimes b) \Delta(a)=\sum_{k} a_{k} \otimes b_{k}$ and notice that

$$
\sum_{k} b_{k} S\left(a_{k}^{*}\right)^{*}=\sum_{k} b_{k} S^{-1}\left(a_{k}\right)=m\left(\iota \otimes S^{-1}\right)\left(\sum_{k} b_{k} \otimes a_{k}\right)=m\left(\iota \otimes S^{-1}\right)\left((b \otimes 1) \Delta^{o p}(a)\right)=\varepsilon(a) b .
$$

Hence

$$
\begin{aligned}
\left((\bar{\pi} \times \pi)(a) r(1), J^{*-1} e_{j} \otimes \pi\left(b^{*}\right) e_{l}\right)=\sum_{i}\left((\bar{\pi} \otimes \pi) \Delta(a)\left(J e_{i} \otimes e_{i}\right), J^{*-1} e_{j} \otimes \pi\left(b^{*}\right) e_{l}\right) \\
\quad=\sum_{i}\left((\bar{\pi} \otimes \pi)(1 \otimes b)(\bar{\pi} \otimes \pi) \Delta(a)\left(J e_{i} \otimes e_{i}\right), J^{*-1} e_{j} \otimes e_{l}\right) \\
\quad=\sum_{i}\left((\bar{\pi} \otimes \pi)((1 \otimes b) \Delta(a))\left(J e_{i} \otimes e_{i}\right), J^{*-1} e_{j} \otimes e_{l}\right) \\
\quad=\sum_{i k}\left(\bar{\pi}\left(a_{k}\right) J e_{i} \otimes \pi\left(b_{k}\right) e_{i}, J^{*-1} e_{j} \otimes e_{l}\right)=\sum_{i k}\left(J \pi S\left(a_{k}^{*}\right) e_{i} \otimes \pi\left(b_{k}\right) e_{i}, J^{*-1} e_{j} \otimes e_{l}\right) \\
\quad=\sum_{i k}\left(J \pi S\left(a_{k}^{*}\right) e_{i}, J^{*-1} e_{j}\right)\left(\pi\left(b_{k}\right) e_{i}, e_{l}\right)=\sum_{i k}\left(e_{j}, \pi S\left(a_{k}^{*}\right) e_{i}\right)\left(\pi\left(b_{k}\right) e_{i}, e_{l}\right) \\
\quad=\sum_{i k}\left(\pi S\left(a_{k}^{*}\right)^{*} e_{j}, e_{i}\right)\left(\pi\left(b_{k}\right) e_{i}, e_{l}\right)=\sum_{k}\left(\pi\left(b_{k}\right) \sum_{i}\left(\pi S\left(a_{k}^{*}\right)^{*} e_{j}, e_{i}\right) e_{i}, e_{l}\right) \\
\quad=\sum_{k}\left(\pi\left(b_{k}\right) \pi S\left(a_{k}^{*}\right)^{*} e_{j}, e_{l}\right)=\left(\pi\left(\sum_{k} b_{k} S\left(a_{k}^{*}\right)^{*}\right) e_{j}, e_{l}\right)=\left(\pi(\varepsilon(a) b) e_{j}, e_{l}\right),
\end{aligned}
$$

as desired. 
Note that for a discrete aqg $(A, \Delta)$, the intrinsic dimension $d(\pi)$ of an irreducible object $\pi \in \operatorname{Rep}_{f}(A, \Delta)$ is then given by

$$
d(\pi)=r^{*} \circ r=\bar{r}^{*} \circ \bar{r}=\operatorname{Tr} \pi(f)=\operatorname{Tr} \pi\left(f^{-1}\right) .
$$

In fact, the latter two expressions can be thought of as the quantum dimension of $\pi$ [30] and gives the intrinsic dimension for any $\pi \in \operatorname{Rep}_{f}(A, \Delta)$. By the Schwarz inequality for the inner product given by $\operatorname{Tr}$ it follows that if $d(\pi)$ equals the dimension of the Hilbert space on which $\pi$ acts, then $\pi(f)=1$. Thus the von Neumann extension of $(A, \Delta)$ is a Kac algebra iff the intrinsic dimension of any finite dimensional representation coincides with the dimension of the Hilbert space on which it acts.

Suppose now that $(A, \Delta)$ is a compact agq with counit $\varepsilon$ and coinverse $S$. We would like to find an expression for the conjugate unitary corepresentation $\bar{V}$ of a non-zero object $V \in \operatorname{Corep}_{f}(A, \Delta)$. To this end, we will use the correspondence between $\operatorname{Rep}_{f}(\hat{A}, \hat{\Delta})$ and $\operatorname{Corep}_{f}(A, \Delta)$ established in Theorem 3.14. Recall that the dual $(\hat{A}, \hat{\Delta})$ of $(A, \Delta)$ is a discrete aqg. Let $\hat{\varepsilon}$ denote its counit, $\hat{S}$ its coinverse, and let $\hat{f} \in M(\hat{A})$ be as in Theorem 2.12 .

Let $V \in A \otimes B(K)$ be a non-zero unitary corepresentation of $(A, \Delta)$ on a finite dimensional Hilbert space $K$. Pick a finite dimensional Hilbert space $\bar{K}$ and a antilinear map $J: K \rightarrow \bar{K}$ such that $J^{*} J=\pi_{V}\left(\hat{f}^{-1}\right) \in B(K)$. Given any invertible antilinear map $J$ we define a linear map $j: B(K) \rightarrow B(\bar{K})$ by $j(x)=J x^{*} J^{-1}$, for all $x \in B(K)$.

Proposition 3.22 Suppose $(A, \Delta)$ is a compact aqg. Let $V$ be a unitary corepresentation of $(A, \Delta)$ on a finite dimensional Hilbert space $K$ with $J^{*} J=\pi_{V}\left(\hat{f}^{-1}\right) \in B(K)$. Define $\bar{V} \in A \otimes B(\bar{K})$ by $\bar{V}=\left(S^{-1} \otimes j\right) V$. Then the following hold:

1. $V\left(1 \otimes J^{*} J\right)=\left(1 \otimes J^{*} J\right)\left(S^{2} \otimes \iota\right) V$,

2. $\bar{V} \in \operatorname{Corep}_{f}(A, \Delta)$,

3. $\overline{\pi_{V}}=\pi_{\bar{V}}$. (Here it is understood that the same $J: K \rightarrow \bar{K}$ is used in both constructions.)

Proof. To prove 1., observe that

$$
\begin{gathered}
(\omega \otimes \iota) V J^{*} J=\pi_{V}(\omega) \pi_{V}\left(\hat{f}^{-1}\right)=\pi_{V}\left(\omega \hat{f}^{-1}\right)=\pi_{V}\left(\hat{f}^{-1}\right) \pi_{V}\left(\hat{f} \omega \hat{f}^{-1}\right) \\
=J^{*} J \pi_{V}\left(\hat{S}^{2}(\omega)\right)=J^{*} J\left(\hat{S}^{2}(\omega) \otimes \iota\right) V=J^{*} J(\omega \otimes \iota)\left(S^{2} \otimes \iota\right) V
\end{gathered}
$$

for all $\omega \in \hat{A}$.

To prove 3., note first that $\hat{S}(\omega)(a)=\omega S(a)$ and $\omega^{*}(a)=\overline{\omega\left(S(a)^{*}\right)}$ for all $a \in A$ and $\omega \in \hat{A}$. Writing $V=\sum_{k} a_{k} \otimes x_{k}$ we compute

$$
\begin{aligned}
\sum_{k} \hat{S}\left(\omega^{*}\right)\left(a_{k}\right) x_{k} & =\sum_{k} \omega^{*} S\left(a_{k}\right) x_{k}=\left(\omega^{*} \otimes \iota\right)(S \otimes \iota) V=\left(\omega^{*} \otimes \iota\right)\left(V^{*}\right) \\
& =\sum_{k} \omega^{*}\left(a_{k}^{*}\right) x_{k}^{*}=\sum_{k} \overline{\omega\left(S\left(a_{k}^{*}\right)^{*}\right)} x_{k}^{*}=\sum_{k} \overline{\omega S^{-1}\left(a_{k}\right)} x_{k}^{*} .
\end{aligned}
$$

Since $(S \otimes \iota) V=V^{*}$, we thus obtain

$$
\begin{aligned}
\overline{\pi_{V}}(\omega) & =J \pi_{V} \hat{S}\left(\omega^{*}\right) J^{-1}=J\left(\hat{S}\left(\omega^{*}\right) \otimes \iota\right) V J^{-1}=\sum_{k} J\left(\hat{S}\left(\omega^{*}\right)\left(a_{k}\right) x_{k}\right) J^{-1}=\sum_{k} J\left(\overline{\omega S^{-1}\left(a_{k}\right)} x_{k}^{*}\right) J^{-1} \\
& =\sum_{k} \omega S^{-1}\left(a_{k}\right) J x_{k}^{*} J^{-1}=(\omega \otimes \iota)\left(S^{-1} \otimes j\right)\left(\sum_{k} a_{k} \otimes x_{k}\right)=(\omega \otimes \iota)\left(S^{-1} \otimes j\right) V=\pi_{\bar{V}}(\omega)
\end{aligned}
$$

for all $\omega \in \hat{A}$, proving 3. Now 3., together with Proposition 3.5 and Proposition 3.21, imply 2., completing the proof.

This suggests the following proposition, which is a formulation more intrinsic to the category $\operatorname{Corep}_{f}(A, \Delta)$. 
Proposition 3.23 Let $(A, \Delta)$ be a compact aqg and $V \in \operatorname{Corep}_{f}(A, \Delta)$ be non-zero. Pick a finite dimensional Hilbert space $\bar{K}$ and any antilinear map $J: K \rightarrow \bar{K}$ such that $V\left(1 \otimes J^{*} J\right)=\left(1 \otimes J^{*} J\right)\left(S^{2} \otimes \iota\right) V$. Then $\bar{V} \in A \otimes B(\bar{K})$ given by $\bar{V}=\left(S^{-1} \otimes j\right) V$ belongs to $\operatorname{Corep}_{f}(A, \Delta)$. It is a conjugate of $V$ with normalized solutions $r \in \operatorname{Hom}(1 \otimes 1, \bar{V} \times V), \bar{r} \in \operatorname{Hom}(1 \otimes 1, V \times \bar{V})$ of the conjugate equations for $V$ and $\bar{V}$ given by

$$
r(1)=\sum_{i} J e_{i} \otimes e_{i} \quad \text { and } \quad \bar{r}(1)=\sum_{i} e_{i} \otimes J^{*-1} e_{i} .
$$

Proof. The irreducible case follows from Proposition 3.21, Theorem 3.14 and the fact that $J^{*} J$ is the unique (up to a positive scalar) positive operator in $B(K)$ with the property $V\left(1 \otimes J^{*} J\right)=\left(1 \otimes J^{*} J\right)\left(S^{2} \otimes \iota\right) V$ [40, 30]. We content ourselves with proving one of the least obvious parts of the proposition, namely that $r$, defined above, belongs to $\operatorname{Hom}(1 \otimes 1, \bar{V} \times V)$. In other words we must show that $r(\omega \otimes \iota)(1 \otimes 1)=(\omega \otimes \iota \otimes \iota)(\bar{V} \times V) r$ or $\omega(1) r(1)=(\omega \otimes \iota \otimes \iota)(\bar{V} \times V) r(1)$ for all $\omega \in \hat{A}$. Write $V=\sum_{k} a_{k} \otimes x_{k} \in A \otimes B(K)$, so $\bar{V}=\sum_{k} S^{-1}\left(a_{k}\right) \otimes J x_{k}^{*} J^{-1}$. For any $\omega \in \hat{A}$ and any elements $e_{j}, e_{r}$ in the chosen orthonormal basis for $K$, we then get

$$
\begin{aligned}
& \left((\omega \otimes \iota \otimes \iota)(\bar{V} \times V) r(1), J^{*-1} e_{j} \otimes e_{r}\right)=\sum_{i}\left((\omega \otimes \iota \otimes \iota)\left(\bar{V}_{12} V_{13}\right)\left(J e_{i} \otimes e_{i}\right), J^{*-1} e_{j} \otimes e_{r}\right) \\
& =\sum_{i k l} \omega\left(S^{-1}\left(a_{k}\right) a_{l}\right)\left(J x_{k}^{*} J^{-1} J e_{i}, J^{*-1} e_{j}\right)\left(x_{l} e_{i}, e_{r}\right)=\sum_{i k l} \omega\left(S^{-1}\left(a_{k}\right) a_{l}\right)\left(J x_{k}^{*} e_{i}, J^{*-1} e_{j}\right)\left(x_{l} e_{i}, e_{r}\right) \\
& =\sum_{i k l} \omega\left(S^{-1}\left(a_{k}\right) a_{l}\right)\left(e_{j}, x_{k}^{*} e_{i}\right)\left(x_{l} e_{i}, e_{r}\right)=\sum_{i k l} \omega\left(S^{-1}\left(a_{k}\right) a_{l}\right)\left(x_{k} e_{j}, e_{i}\right)\left(x_{l} e_{i}, e_{r}\right) \\
& =\sum_{k l} \omega\left(S^{-1}\left(a_{k}\right) a_{l}\right)\left(x_{l} \sum_{i}\left(x_{k} e_{j}, e_{i}\right) e_{i}, e_{r}\right)=\sum_{k l} \omega\left(S^{-1}\left(a_{k}\right) a_{l}\right)\left(x_{l} x_{k} e_{j}, e_{r}\right) \\
& =\sum_{k l} \omega S^{-1}\left(S\left(a_{l}\right) a_{k}\right)\left(x_{l} x_{k} e_{j}, e_{r}\right)=\sum_{k l} \omega S^{-1}\left(a_{l}^{*} a_{k}\right)\left(x_{l}^{*} x_{k} e_{j}, e_{r}\right) \\
& =\left(\omega S^{-1} \otimes \omega_{e_{j}, e_{r}}\right)\left(V^{*} V\right)=\left(\omega \otimes \omega_{e_{j}, e_{r}}\right)(1 \otimes 1)=\omega(1)\left(e_{j}, e_{r}\right) \\
& =\sum_{i} \omega(1)\left(J e_{i}, J^{*-1} e_{j}\right)\left(e_{i}, e_{r}\right)=\left(\omega(1) r(1), J^{*-1} e_{j} \otimes e_{r}\right),
\end{aligned}
$$

where

$$
\omega_{e_{j}, e_{r}}: B(K) \rightarrow \mathbb{C}, \quad x \mapsto\left(x e_{j}, e_{r}\right)
$$

Thus

$$
(\omega \otimes \iota \otimes \iota)(\bar{V} \times V) r(1)=\omega(1) r(1)
$$

completing the proof.

REMARK 3.24 In the last two results, the conjugates can be expressed alternatively in terms of the unitary antipode $R$ and an antiunitary $J$ [5].

\section{Cocommutative Algebraic Quantum Groups vs. Compact Groups}

Definition 4.1 Let $(A, \Delta)$ be an aqg. The intrinsic group $G$ of $(A, \Delta)$ is the following subgroup of the unitaries in the multiplier algebra $M(A)$ :

$$
G=\left\{g \in M(A) \mid \Delta g=g \otimes g, g^{*} g=g g^{*}=1\right\} .
$$

Since the extended comultiplication $\Delta: M(A) \rightarrow M(A \otimes A)$ is a unital $*$-homomorphism and the algebra $M(A)$ is associative, the set $G$ is indeed a group. It is easy to see that $\varepsilon(g)=1$ and $S(g)=g^{-1}=g^{*}$, for any $g \in G$. 
REMARK 4.2 It can be shown that any bounded group-like element of $M(A)$ is automatically unitary with $S(g)=g^{*}$. A proof of this may be found in [20] and [11], but the above definition suffices for our purposes.

Lemma 4.3 Let $(A, \Delta)$ be a discrete aqg. Equipped with the product topology, $M(A)=\prod_{i \in I} B\left(H_{i}\right)$ is a complete locally convex topological vector space and $A$ is dense in $M(A)$.

Proof. Since the blocks $B\left(H_{i}\right)$ are Banach spaces, the functions $\left\|p_{i}(\cdot)\right\|$ form a separating family of seminorms which induces the product topology. Completeness is obvious by semisimplicity of the $B\left(H_{i}\right), i \in I$. To see that $A$ is dense in $M(A)$, let $x \in M(A)$ and consider the net $\left(x_{\lambda}\right)_{\lambda \in \Lambda}$ in $A$ given by $x_{\lambda}=\oplus_{i \in \lambda} p_{i}(x)$, where $\Lambda$ is the collection of finite subsets of $I$ directed by inclusions. Then clearly $x_{\lambda} \rightarrow x$.

Proposition 4.4 If $(A, \Delta)$ is a discrete aqg, its intrinsic group $G$ is a compact topological group w.r.t. the product topology on $M(A)$.

Proof. Note that a net $\left(g_{\lambda}\right)$ converges to $g$ in $G$ iff $p_{i}\left(g_{\lambda}\right) \rightarrow p_{i}(g)$ in norm, for all $i \in I$. By Tychonov's theorem it suffices to show that $G$ is closed in $\prod_{i \in I} U\left(B\left(H_{i}\right)\right)$. Given a net $\left(g_{\lambda}\right)$ in $G$ which converges to $a \in M(A)$, we must show that $\Delta a=a \otimes a$. Let $i, j \in I$ and consider the finite dimensional non-degenerate $*$-representation $\left(p_{i} \otimes p_{j}\right) \Delta: A \rightarrow B\left(H_{i} \otimes H_{j}\right)$. By Proposition 2.10, we may write

$$
\left(p_{i} \otimes p_{j}\right) \Delta \simeq \bigoplus_{k \in I} N_{i j}^{k} p_{k}
$$

Since both these expressions are non-degenerate $*$-representations, they extend to equivalent unital $*$-representations of $M(A)$, which we now identify. Therefore

$$
\begin{gathered}
\left(p_{i} \otimes p_{j}\right) \Delta a=\bigoplus_{k \in I} N_{i j}^{k} p_{k}(a)=\lim _{\lambda} \bigoplus_{k \in I} N_{i j}^{k} p_{k}\left(g_{\lambda}\right)=\lim _{\lambda}\left(p_{i} \otimes p_{j}\right) \Delta g_{\lambda} \\
=\lim _{\lambda} p_{i}\left(g_{\lambda}\right) \otimes p_{j}\left(g_{\lambda}\right)=p_{i}(a) \otimes p_{j}(a)=\left(p_{i} \otimes p_{j}\right)(a \otimes a),
\end{gathered}
$$

thus $\Delta a=a \otimes a$ and $G$ is closed.

REMARK 4.5 Note furthermore that in the proof of the proposition we have shown that

$$
\Delta: M(A)=\prod_{i \in I} B\left(H_{i}\right) \rightarrow M(A \otimes A)=\prod_{i, j \in I} B\left(H_{i} \otimes H_{j}\right)
$$

is continuous w.r.t. the product topologies.

Proposition 4.6 Suppose $(A, \Delta)$ is a discrete aqg with intrinsic group $G$. Let $\pi$ be a non-degenerate *representation of $A$ on a finite dimensional Hilbert space $K$. Define a map $u_{\pi}: G \rightarrow B(K)$ by

$$
u_{\pi}: g \mapsto \pi(g),
$$

where the extension of $\pi$ to $M(A)$ is understood. Then $u_{\pi}$ is strongly continuous and $D:(K, \pi) \mapsto\left(K, u_{\pi}\right)$ together with the identity map on the morphisms is a faithful and tensor functor from $\operatorname{Rep}_{f}(A, \Delta)$ to the category $\operatorname{Rep}_{f} G$ of finite dimensional continuous representations of $G$. 
Proof. Continuity of $u_{\pi}$ w.r.t. the topology on $G$ is obvious. As $\pi: M(A) \rightarrow B(K)$ is a unital $*$-homomorphism, clearly $\left(K, u_{\pi}\right) \in \operatorname{Rep} G$. Functoriality and faithfulness of $D$ are obvious. Monoidality follows from the calculation

$$
D\left(\pi_{1} \times \pi_{2}\right)(g)=\left(\pi_{1} \otimes \pi_{2}\right) \Delta g=\pi_{1}(g) \otimes \pi_{2}(g)=u_{\pi_{1}}(g) \otimes u_{\pi_{2}}(g)=\left(u_{\pi_{1}} \times u_{\pi_{2}}\right)(g)
$$

for all $g \in G$ and $\pi_{i} \in \operatorname{Rep}(A, \Delta)$. Every $\pi \in \operatorname{Rep}(A, \Delta)$ is equivalent to a direct sum of the representations $p_{i}$ by Proposition 2.10. Each $u_{p_{i}}$ is (strongly) continuous and therefore the direct sum is strongly continuous.

Thus far we have not assumed $(A, \Delta)$ to be cocommutative. The following characterization of cocommutativity will be crucial for proving that $D$ gives rise to an equivalence of categories.

TheOREm 4.7 A discrete aqg $(A, \Delta)$ is cocommutative iff

$$
\overline{\operatorname{span}_{\mathbb{C}}\{g \mid g \in G\}}=M(A) .
$$

REMARK 4.8 This theorem implies that

$$
\operatorname{span}_{\mathbb{C}}\left\{p_{i}(g) \mid g \in G\right\}=B\left(H_{i}\right)
$$

for every $i \in I$ and in particular that $A=\operatorname{span}_{\mathbb{C}}\left\{g I_{i} \mid i \in I, g \in G\right\}$. These results are rigorous formulations of the heuristic idea that a cocommutative aqg is 'spanned by its grouplike elements'. Before we give the proof of Theorem 4.7 we show that it leads to the desired equivalence of categories.

In what follows we fix a cocommutative discrete aqg $(A, \Delta)$ where $A=\oplus_{i \in I} B\left(H_{i}\right)$ and let $G$ denote its intrinsic group. For every $i \in I, u_{i}: g \mapsto p_{i}(g) \in B\left(H_{i}\right)$ is a continuous unitary representation of $G$. By Theorem 4.7, the span of $p_{i}(g), g \in G$ is dense in $B\left(H_{i}\right)$, thus $u_{i}$ is irreducible. This defines a map $\gamma: I \rightarrow I_{G}$.

Proposition 4.9 The map $\gamma$ is a bijection.

Proof. If there is a unitary $V: H_{i} \rightarrow H_{j}$ such that $V p_{i}(g)=p_{j}(g) V$ for all $g \in G$ then $V p_{i}(x)=p_{j}(x) V$ for all $x \in \operatorname{span}_{\mathbb{C}}\{g \mid g \in G\}$. Since these $x$ are dense in $M(A)$ by Theorem 4.7 and the $p_{i}$ are continuous we conclude that $V p_{i}(x)=p_{j}(x) V$ for all $x \in M(A)$, thus $i=j$ so that $\gamma: I \rightarrow I_{G}$ is injective.

Obviously if $g \neq e$ there is an $i \in I$ such that $p_{i}(g) \neq 1_{B\left(H_{i}\right)}$. Since the category $\operatorname{Rep}_{f}(A, \Delta)$ is monoidal and has conjugates, $\gamma(I) \subset I_{G}$ is closed w.r.t. conjugation and tensor products and reduction. The surjectivity of $\gamma$ now follows from the following well known group theoretical fact.

Lemma 4.10 Let $G$ be a compact group and let $J \subset I_{G}$ be closed w.r.t. conjugation and tensor products and reduction. If $J$ separates points on $G$ then $J=I_{G}$.

Proof. For every equivalence class $i \in I_{G}$ pick a representative $u_{i}$. The assumptions on $J$ imply that the span of the matrix elements $\left(u_{j}\right)_{n m}, j \in J$ is a unital $*$-subalgebra of $C(G)$. Since it separates the points of $G$ it is dense in $C(G)$ by the Stone-Weierstrass theorem and therefore dense in $L^{2}(G, \mu)$, where $\mu$ is the Haar measure. If there were a $k \in I_{G} \backslash J$ then by the Peter-Weyl theorem the matrix elements of $u_{k}$ would be orthogonal to the dense subspace of $L^{2}(G, \mu)$ generated by the $\left(u_{j}\right)_{n m}, j \in J$, which is a contradiction.

TheOREM 4.11 Let $(A, \Delta)$ be a cocommutative discrete aqg and $G$ its intrinsic group. Then the functor $D$ : $\operatorname{Rep}(A, \Delta) \rightarrow \operatorname{Rep} G$ induces a canonical equivalence of tensor $*$-categories:

$$
\operatorname{Rep}_{f}(A, \Delta) \stackrel{\otimes}{\simeq} \operatorname{Rep}_{f} G .
$$


Proof. In view of Proposition 4.6 it only remains to prove that the functor $D$ is full and essentially surjective. By Proposition 2.10 the category $\operatorname{Rep}_{f}(A, \Delta)$ is semisimple, and for a compact group $G$ the semisimplicity of $\operatorname{Rep}_{f} G$ is well known. Recall from Section 2.1 that a faithful functor between semisimple categories is full if and only if it maps simple objects to simple objects and non-isomorphic simple objects have non-isomorphic images. The first property was used to define the map $\gamma$ of Proposition 4.9, and the second is the injectivity of $\gamma$. Finally, essential surjectivity of $D$ is expressed by surjectivity of $\gamma$. Since $D$ is monoidal it gives rise to an equivalence of tensor categories by [31].

Now we prove the characterization of cocommutative discrete aqg used above.

Proof of Theorem 4. . Clearly, if the closure of $\operatorname{span}_{\mathbb{C}}\{g \mid g \in G\}$ is $M(A)$, then by linearity and continuity of $\Delta: M(A) \rightarrow M(A \otimes A)$, we see that $(A, \Delta)$ is cocommutative. We prove the converse direction. By Lemma 4.3 it suffices to show that any $x \in A$ is the limit of linear combinations of elements of $G$. Fix $x \in A$. For any aqg $(A, \Delta)$ there exists a linear inclusion $Q: A \hookrightarrow \hat{A}_{r}^{*}$ determined by

$$
Q(b) \hat{\pi}_{r}(\hat{a})=\hat{a}(b)=\varphi(b a)
$$

for all $a, b \in A$. To see this, to any $a, b \in A$ we can choose an element $c \in A$ such that $\hat{c} \hat{b}^{*}=\hat{b}^{*}$. (Such $c$ can be obtained as inverse Fourier transform of a local unit for ${\hat{b^{*}}}^{*}$ in $\hat{A}$.) Now we observe that

$$
Q(b) \hat{\pi}_{r}(\hat{a})=\varphi\left(\left(b^{*}\right)^{*} a\right)=\hat{\psi}\left(\hat{b}^{*} \hat{a}\right)=\hat{\psi}\left(\hat{c}{\hat{b}^{*}}^{*} \hat{a}\right)=\hat{\psi}\left(\hat{b}^{*} \hat{a} \hat{\rho}(\hat{c})\right)=\left(\hat{\pi}_{r}(\hat{a}) \hat{\Lambda}(\hat{\rho}(\hat{c})), \hat{\Lambda}\left(\hat{b}^{*}\right)\right),
$$

so $Q(b)=\left(\cdot \hat{\Lambda}(\hat{\rho}(\hat{c})), \hat{\Lambda}\left(\hat{b}^{*}\right)\right)$. Now $\hat{A}_{r}$ is a unital commutative $C^{*}$-algebra. Let $Y$ be the set of $*$-characters on $\hat{A}_{r}$. Gelfand's theorem tells us that $Y$ is a compact Hausdorff space and that the map $\Gamma: \hat{A}_{r} \rightarrow C(Y)$ given by $\Gamma(a)(y)=y(a)$, for all $a \in \hat{A}_{r}$ and $y \in Y$, is a unital $*$-isomorphism from $\hat{A}_{r}$ to the $C^{*}$-algebra $C(Y)$ of continuous functions on $Y$. Thus $Q(x) \Gamma^{-1} \in C(Y)^{*}$. By the Krein-Milman theorem for probability measures 27, Theorem 2.5.4] any element of $C(Y)^{*}$ is a $w^{*}$-limit of linear combinations of Dirac measures $\delta_{y} \in C(Y)^{*}$. Hence we may write

$$
Q(x) \Gamma^{-1}=\lim _{\lambda} \sum_{k} c_{\lambda k} \delta_{y_{\lambda k}}
$$

for some $c_{\lambda k} \in \mathbb{C}$ and $y_{\lambda k} \in Y$. Thus we get

$$
\begin{aligned}
\hat{a}(x) & =Q(x) \hat{\pi}_{r}(\hat{a})=Q(x) \Gamma^{-1} \Gamma \hat{\pi}_{r}(\hat{a})=\lim _{\lambda} \sum_{k} c_{\lambda k} \delta_{y_{\lambda k}} \Gamma \hat{\pi}_{r}(\hat{a}) \\
& =\lim _{\lambda} \sum_{k} c_{\lambda k} \Gamma \hat{\pi}_{r}(\hat{a})\left(y_{\lambda k}\right)=\lim _{\lambda} \sum_{k} c_{\lambda k} y_{\lambda k} \hat{\pi}_{r}(\hat{a})
\end{aligned}
$$

for all $a \in A$. Now the $y_{\lambda k} \hat{\pi}_{r}: \hat{A} \rightarrow \mathbb{C}$ are unital $*$-homomorphisms, so we may define $g_{\lambda k} \in M(A)$ by

$$
g_{\lambda k}=\left(\iota \otimes y_{\lambda k} \hat{\pi}_{r}\right) U
$$

where $U \in M(A \otimes \hat{A})$ is the universal corepresentation of $(A, \Delta)$. Since $U$ is unitary, so are the elements $g_{\lambda k}$ and moreover,

$$
\begin{aligned}
\Delta g_{\lambda k} & =\Delta\left(\iota \otimes y_{\lambda k} \hat{\pi}_{r}\right) U=\left(\iota \otimes \iota \otimes y_{\lambda k} \hat{\pi}_{r}\right)(\Delta \otimes \iota) U=\left(\iota \otimes y_{\lambda k} \hat{\pi}_{r}\right)\left(U_{13} U_{23}\right) \\
& =\left(\iota \otimes y_{\lambda k} \hat{\pi}_{r}\right) U \otimes\left(\iota \otimes y_{\lambda k} \hat{\pi}_{r}\right) U=g_{\lambda k} \otimes g_{\lambda k},
\end{aligned}
$$

thus all $g_{\lambda k} \in G$.

Now for any $\xi \in B\left(H_{i}\right)^{\prime}$ and $i \in I$ observe that $\xi p_{i} \in \hat{A}$ since $\varphi$ is, up to a factor $d_{i}$, the trace on $B\left(H_{i}\right)$. Thus by Theorem 3.4 we have $\left(\xi p_{i} \otimes \iota\right) U=\xi p_{i}$. Hence by the previous formulae we get

$$
\begin{aligned}
\xi p_{i}(x) & =\lim _{\lambda} \sum_{k} c_{\lambda k} y_{\lambda k} \hat{\pi}_{r}\left(\xi p_{i}\right)=\lim _{\lambda} \sum_{k} c_{\lambda k} y_{\lambda k} \hat{\pi}_{r}\left(\xi p_{i} \otimes \iota\right) U \\
& =\lim _{\lambda} \sum_{k} c_{\lambda k} \xi p_{i}\left(\iota \otimes y_{\lambda k} \hat{\pi}_{r}\right) U=\lim _{\lambda} \sum_{k} c_{\lambda k} \xi p_{i}\left(g_{\lambda k}\right)=\lim _{\lambda} \xi p_{i}\left(\sum_{k} c_{\lambda k} g_{\lambda k}\right) .
\end{aligned}
$$


Since this is true for all $i \in I$ and $\xi \in B\left(H_{i}\right)^{\prime}$, we thus get

$$
x=\lim _{\lambda} \sum_{k} c_{\lambda k} g_{\lambda k}
$$

as desired.

This concludes the proof of Theorem 4.7.

Corollary 4.12 For every $i \in I$ we have $\operatorname{span}_{\mathbb{C}}\left\{p_{i}(g) \mid g \in G\right\}=B\left(H_{i}\right)$.

Proof. First we show that for $0 \neq a \in A$, there exist $g \in G$ such that $\varphi(g a) \neq 0$. Thus let $0 \neq a \in A$. Then there exists $x \in A$ such that $\hat{a}(x)=\varphi(x a) \neq 0$, thus $\hat{a}=\varphi(\cdot a) \neq 0$. Furthermore, there exists a finite set $\Lambda \subset I$ such that

$$
\hat{a}(b)=\varphi(b a)=\sum_{i \in \Lambda} d_{i} T r_{i}\left(p_{i}(b a)\right)
$$

for all $b \in M(A)$. Since the product in $M(A)$ is continuous, we thus see that $\hat{a}: M(A) \rightarrow \mathbb{C}$ is continuous. Thus by Theorem 4.7 there exists $g \in G$ such that $\varphi(g a) \neq 0$.

Assume now that $\operatorname{span}_{\mathbb{C}}\left\{p_{i}(g) \mid g \in G\right\} \neq B\left(H_{i}\right)$. Then there exists a non-zero $a \in B\left(H_{i}\right)$ such that $\operatorname{Tr}_{B\left(H_{i}\right)}\left(p_{i}(g) a\right)=0$ for all $g \in G$. Since $\varphi\left\lceil B\left(H_{i}\right)=d_{i} \operatorname{Tr}_{B\left(H_{i}\right)}\right.$, this is a contradiction.

Let $G$ be a compact group and consider a unitary representation $u: G \rightarrow B(K)$ of $G$ on some finite dimensional Hilbert space $K$. Since $u$ is continuous (here with respect to the norm), we have $u \in C(G, B(K)$ ). Since $C(G, B(K)) \cong C(G) \otimes B(K)$, we may thus regard $u$ as a unitary element $\tilde{V}$ of the unital $*$-algebra $C(G) \otimes B(K)$. Now $u: G \rightarrow B(K)$ being a representation simply means that $(\Delta \otimes \iota) \tilde{V}=\tilde{V}_{13} \tilde{V}_{23}$, where $\Delta: C(G) \rightarrow C(G \times G) \cong C(G) \otimes C(G)$ is given by $\Delta(a)\left(g, g^{\prime}\right)=a\left(g g^{\prime}\right)$, for all $a \in C(G)$ and $g, g^{\prime} \in G$. Thus $\tilde{V}$ is a unitary corepresentation of the commutative compact quantum group $(C(G), \Delta)$ in the sense of Woronowicz. It is easy to see that one may recover $u$ from $\tilde{V}$ by $u(g)=\left(\delta_{g} \otimes \iota\right) \tilde{V}$, for $g \in G$.

THEOREM 4.13 Let $(A, \Delta)$ be a cocommutative discrete aqg, so $(\hat{A}, \hat{\Delta})$ is a commutative compact aqg. Let $G$ be the compact group such that $(C(G), \Delta) \cong\left(\hat{A}_{r}, \hat{\Delta}_{r}\right)$. For any $V \in \operatorname{Corep}_{f}(\hat{A}, \hat{\Delta})$, let $\tilde{V} \in C(G) \otimes B(K)$ be the unitary corepresentation of $(C(G), \Delta)$ given by $\tilde{V}=\left(\hat{\pi}_{r} \otimes \iota\right) V$. Then there exists an isomorphism from $G$ to the intrinsic group of $(A, \Delta)$ given by

$$
g \mapsto \tilde{g}=\left(\iota \otimes \delta_{g} \hat{\pi}_{r}\right) U \in M(A),
$$

where $U$ is the universal corepresentation of $(A, \Delta)$. Moreover, the equivalence

$$
\operatorname{Corep}_{f}(\hat{A}, \hat{\Delta}) \stackrel{P}{\longrightarrow} \operatorname{Rep}_{f}(A, \Delta) \stackrel{D_{f}}{\longrightarrow} \operatorname{Rep}_{f} G
$$

given by the composition

$$
V \mapsto \pi_{V} \mapsto u_{\pi_{V}}=: u_{V},
$$

with $P$ and $D_{f}$ established in Theorem 3.14 and Theorem 4.11, is described more directly by

$$
u_{V}(\tilde{g})=\left(\delta_{g} \otimes \iota\right) \tilde{V},
$$

for all $g \in G$.

Proof. From the proof of Theorem 4.7, it is easily seen that $g \mapsto \tilde{g}$ is indeed a homeomorphism from $G$ to the intrinsic group of $(A, \Delta)$ which also preserves the products. Let $g \in G$. We proceed to show that 
$u_{V}(\tilde{g})=\left(\delta_{g} \otimes \iota\right) \tilde{V}$. Since $V \in \hat{A} \otimes B(K)$ we may write $V=\sum_{k} \omega_{k} \otimes x_{k}$ for $\omega_{k} \in \hat{A}$ and $x_{k} \in B(K)$. Then

$$
\begin{aligned}
& u_{V}(\tilde{g})=u_{V}\left(\left(\iota \otimes \delta_{g} \hat{\pi}_{r}\right) U\right)=\pi_{V}\left(\left(\iota \otimes \delta_{g} \hat{\pi}_{r}\right) U\right) \\
& \quad=\left(\left[\left(\iota \otimes \delta_{g} \hat{\pi}_{r}\right) U\right] \otimes \iota\right) V=\sum_{k}\left[\left(\iota \otimes \delta_{g} \hat{\pi}_{r}\right) U\right]\left(\omega_{k}\right) x_{k}=\sum_{k}\left(\omega_{k} \otimes \delta_{g} \hat{\pi}_{r}\right) U x_{k} \\
& \quad=\sum_{k} \delta_{g} \hat{\pi}_{r}\left(\left(\omega_{k} \otimes \iota\right) U\right) x_{k}=\sum_{k} \delta_{g} \hat{\pi}_{r}\left(\omega_{k}\right) x_{k}=\left(\delta_{g} \hat{\pi}_{r} \otimes \iota\right)\left(\sum_{k} \omega_{k} \otimes x_{k}\right)=\left(\delta_{g} \hat{\pi}_{r} \otimes \iota\right) V=\left(\delta_{g} \otimes \iota\right) \tilde{V},
\end{aligned}
$$

as claimed.

\section{From Concrete Tensor Categories to Discrete Quantum Groups}

The strategy we adopt in this section was been pursued by J.E. Roberts [29] in the discrete Kac algebra case, and has then been adapted to the more algebraic setting of discrete aqg $(A, \Delta)$ by the authors.

\subsection{Natural Transformations of $*$-Preserving Functors}

Proposition 5.1 Let $\mathcal{D}, \mathcal{K}$ be $*$-categories and $F: \mathcal{D} \rightarrow \mathcal{K}$ a $*$-preserving functor. Let $\mathrm{Nat} F$ be the set of all natural transformations from $F$ to itself, viz.

$$
\text { Nat } F=\left\{b=\left(b_{X} \in \operatorname{End} F(X), X \in \mathcal{D}\right) \mid F(s) \circ b_{X}=b_{Y} \circ F(s) \forall s: X \rightarrow Y\right\} .
$$

Then with the following pointwise operations Nat $F$ is a unital $*$-algebra:

$$
\begin{aligned}
\left(\lambda b+\lambda^{\prime} b^{\prime}\right)_{X} & =\lambda b_{X}+\lambda^{\prime} b_{X}^{\prime}, \\
\left(b b^{\prime}\right)_{X} & =b_{X} \circ b_{X}^{\prime}, \\
\left(b^{*}\right)_{X} & =\left(b_{X}\right)^{*}, \\
\mathbf{1}_{X} & =i d_{F(X)},
\end{aligned}
$$

for $b, b^{\prime} \in \operatorname{Nat} F, \lambda, \lambda^{\prime} \in \mathbb{C}$ and $X \in \mathcal{D}$.

Proof. If $s \in \operatorname{Mor}(X, Y)$, then

$$
F(s) \circ\left(b^{*}\right)_{X}=\left(b_{X} \circ F\left(s^{*}\right)\right)^{*}=\left(F\left(s^{*}\right) \circ b_{Y}\right)^{*}=\left(b^{*}\right)_{Y} \circ F(s),
$$

where we have used $F\left(s^{*}\right)=F(s)^{*}$. Thus $b^{*} \in$ Nat $F$. Similarly, we get $b b^{\prime}, \lambda b+\lambda^{\prime} b^{\prime} \in$ Nat $F$, so with these operations Nat $F$ is a $*$-algebra. Obviously 1 is a unit for Nat $F$.

Definition 5.2 Let $B$ be $a$ *-algebra and $\mathcal{K} a *$-category. Then the category $\operatorname{Rep}_{\mathcal{K}} B$ of 'representations of $B$ in $\mathcal{K}$ ' has as objects the pairs $\left(X, \pi_{X}\right)$, where $X \in \operatorname{Obj} \mathcal{K}$ and $\pi_{X}$ is a non-degenerate $*$-homomorphism of $B$ into End $X$. The morphisms are given by

$$
\operatorname{Mor}_{\operatorname{Rep}_{\mathcal{K}} B}\left(\left(X, \pi_{X}\right),\left(Y, \pi_{Y}\right)\right)=\left\{s \in \operatorname{Mor}_{\mathcal{K}}(X, Y) \mid s \circ \pi_{X}(b)=\pi_{Y}(b) \circ s \quad \forall b \in B\right\} .
$$

Note that if $\mathcal{K}=\mathcal{H}$, the category of finite dimensional Hilbert spaces, we have End $H=B(H)$. Thus our definition reduces to the usual notion of finite dimensional non-degenerate $*$-representations of $B$ : $\operatorname{Rep}_{\mathcal{H}} B=$ $\operatorname{Rep}_{f} B$.

Proposition 5.3 Let $\mathcal{C}, \mathcal{K}$ be $*$-categories and $E: \mathcal{C} \rightarrow \mathcal{K}$ a $*$-preserving functor. Define a unital $*$-algebra $B=$ Nat $E$. There is a $*$-preserving functor $F: \mathcal{C} \rightarrow \operatorname{Rep}_{\mathcal{K}} B$ satisfying $K \circ F=E$, where $K: \operatorname{Rep}_{\mathcal{K}} B \rightarrow$ $\mathcal{K},\left(X, \pi_{X}\right) \mapsto X$ is the forgetful functor. 
Proof. We set $F(X)=\left(E(X), \pi_{X}\right)$ for $X \in \operatorname{Obj} \mathcal{C}$ and $F(s)=E(s)$ for the morphisms of $\mathcal{C}$. Here the $*-$ representation $\pi_{X}$ is defined by

$$
\pi_{X}: \quad B \rightarrow \operatorname{End} X, \quad b \mapsto b_{X} .
$$

That $F$ really is a functor follows from

$$
F(s) \circ \pi_{X}(b)=E(s) \circ b_{X}=b_{Y} \circ E(s)=\pi_{Y}(b) \circ F(s) \quad \forall s: X \rightarrow Y \forall b \in B,
$$

where we used $b \in B=$ Nat $E$.

Proposition 5.4 Let $\mathcal{D}$ be a semisimple $*$-category, $\mathcal{K}$ a $*$-category and $F: \mathcal{D} \rightarrow \mathcal{K}$ a $*$-preserving functor. Then there is an isomorphism

$$
\psi_{F}: \text { Nat } F \rightarrow \prod_{i \in I_{\mathcal{D}}} \text { End } F\left(X_{i}\right)
$$

of *-algebras.

Proof. We define a map $\psi_{F}:$ Nat $F \rightarrow \prod_{i \in I_{\mathcal{D}}}$ End $F\left(X_{i}\right)$ by

$$
\psi_{F}(b)=\prod_{i \in I_{\mathcal{D}}} b_{X_{i}}
$$

for all $b \in$ Nat $F$. Clearly $\psi_{F}$ is a unital $*$-algebra homomorphism. We first show that it is injective. Suppose $\psi_{F}(b)=0$, so $b_{X_{i}}=0$ for all $i \in I_{\mathcal{D}}$. Let $X \in \mathcal{D}$. We must show that $b_{X}=0$. Recall that, for any $i \in I_{\mathcal{D}}, \operatorname{Mor}\left(X_{i}, X\right)$ is a Hilbert space with the inner product $(s, t) \operatorname{id}_{X_{i}}=t^{*} s$ and that every $s \in \operatorname{Mor}\left(X_{i}, X\right)$ is a multiple of an isometry. Let $s_{i \alpha} \in \operatorname{Mor}\left(X_{i}, X\right), i \in I_{\mathcal{C}}, \alpha=1, \ldots, \operatorname{dim} \operatorname{Mor}\left(X_{i}, X\right)$ be an orthonormal basis w.r.t. this inner product satisfying $\sum_{i \alpha} s_{i \alpha} \circ s_{i \alpha}^{*}=\operatorname{id}_{X}$. Hence

$$
b_{X}=b_{X} \circ F\left(\mathrm{id}_{X}\right)=\sum_{i \alpha} b_{X} \circ F\left(s_{i} \circ s_{i}^{*}\right)=\sum_{i \alpha} F\left(s_{i}\right) \circ b_{X_{i}} \circ F\left(s_{i}^{*}\right)=0,
$$

and injectivity of $\psi_{F}$ follows.

Next we prove surjectivity of $\psi_{F}$. Given $\left(b_{i} \in \operatorname{End} F\left(X_{i}\right), i \in I_{\mathcal{D}}\right)$ we need to construct $b \in$ Nat $F$ such that $\psi_{F}(b)=\prod_{i \in I_{\mathcal{D}}} b_{i}$. Thus let $X \in \mathcal{D}$. As above, pick a basis of isometries $s_{i \alpha} \in \operatorname{Mor}\left(X_{i}, X\right), i \in I_{\mathcal{D}}$, with $\sum_{i \alpha} s_{i \alpha} \circ s_{i \alpha}^{*}=\operatorname{id}_{X}$. Define $b_{X} \in$ End $F(X)$ by

$$
b_{X}=\sum_{i \alpha} F\left(s_{i \alpha}\right) \circ b_{i} \circ F\left(s_{i \alpha}^{*}\right) .
$$

Another choice of orthonormal bases $\left(s_{i \alpha}\right)$ in $\operatorname{Mor}\left(X_{i}, X\right), i \in I_{\mathcal{D}}$ does not affect $b_{X}$ since any two such bases are related by an orthogonal transformation. It remains to show that $b \in \operatorname{Nat} F$, viz. $F(s) \circ b_{X}=b_{Y} \circ F(s) \forall s$ : $X \rightarrow Y$. To this purpose consider $u \in \operatorname{Mor}(X, Y)$. Pick isometries $t_{j \beta} \in \operatorname{Mor}\left(Y_{j}, Y\right)$, with $\sum_{j \beta} t_{j \beta} \circ t_{j \beta}^{*}=\mathrm{id}_{Y}$, and define $b_{Y} \in$ End $F(Y)$ by

$$
b_{Y}=\sum_{j \beta} F\left(t_{j \beta}\right) \circ b_{j} \circ F\left(t_{j \beta}^{*}\right) .
$$

Then

$$
F(u) \circ b_{X}=\sum_{i \alpha} F\left(u \circ s_{i \alpha}\right) \circ b_{i} \circ F\left(s_{i \alpha}^{*}\right)=\sum_{i \alpha, j \beta} F\left(t_{j \beta} \circ t_{j \beta}^{*} \circ u \circ s_{i \alpha}\right) \circ b_{i} \circ F\left(s_{i \alpha}^{*}\right) .
$$

But $t_{j \beta}^{*} \circ u \circ s_{i \alpha} \in \operatorname{Mor}\left(X_{i}, X_{j}\right)$ is zero unless $i=j$ and otherwise a scalar multiple of $\operatorname{id}_{X_{i}}$. Hence

$F(u) \circ b_{X}=\sum_{i \alpha \beta} F\left(t_{i \beta} \circ\left(t_{i \beta}^{*} \circ u \circ s_{i \alpha}\right)\right) \circ b_{i} \circ F\left(s_{i \alpha}^{*}\right)=\sum_{i \alpha, j \beta} F\left(t_{j \beta}\right) \circ b_{j} \circ F\left(t_{j \beta}^{*} \circ u \circ s_{i \alpha} \circ s_{i \alpha}^{*}\right)=b_{Y} \circ F(u)$. 
The last identity in this equation follows by precisely the same sort of argument as used before. This proves the naturality of the family $b \in \operatorname{Nat} F$. Obviously we have $b_{X_{i}}=b_{i}$ for all $i \in I_{\mathcal{D}}$. Thus $\psi_{F}(b)=\prod_{i \in I_{\mathcal{D}}} b_{i}$, which proves surjectivity of $\psi_{F}$.

The isomorphism $\psi_{F}$ depends on the chosen family $X_{i}, i \in I$ of irreducibles in $\mathcal{C}$, but is canonical once the latter is fixed. Thus there is no risk in suppressing it and writing Nat $F=\prod_{i \in I_{\mathcal{D}}}$ End $F\left(X_{i}\right)$, as will be done in the sequel.

EXAMPLE 5.5 The simplest application of this proposition is to the identity $\mathcal{D} \rightarrow \mathcal{D}$, when we get

$$
\operatorname{Nat}(I d) \cong \prod_{i \in I_{\mathcal{D}}} \mathbb{C}
$$

Examples of natural transformations of the identity are the 'twists' appearing in the context of braided tensor categories.

EXAMPLE 5.6 Suppose $\mathcal{D}$ is a tensor $*$-category with tensor product $\otimes$. A less trivial application of the proposition is the case of the tensor functor $F=\otimes: \mathcal{D} \times \mathcal{D} \rightarrow \mathcal{D}$, where $\mathcal{D} \times \mathcal{D}$ is the product $*$-category. Note that the isomorphism classes of irreducible objects in $\mathcal{D} \times \mathcal{D}$ are precisely $I_{\mathcal{D}} \times I_{\mathcal{D}}$. By semisimplicity of $\mathcal{D}$ we have a finite decomposition

$$
X_{i} \otimes X_{j} \cong \bigoplus_{k \in I_{\mathcal{D}}} N_{i j}^{k} X_{k}
$$

for any $i, j \in I_{\mathcal{D}}$ and thus $\operatorname{End}\left(X_{i} \otimes X_{j}\right) \cong \bigoplus_{k \in I_{\mathcal{D}}} M_{N_{i j}^{k}}(\mathbb{C})$. We therefore obtain

$$
\mathrm{Nat} \otimes \cong \prod_{\substack{i, j, k \in I_{\mathcal{D}} \\ N_{i j}^{k} \neq 0}} M_{N_{i j}^{k}}(\mathbb{C}) .
$$

The following example is a continuation of Example 5.5.

Example 5.7 Suppose $\mathcal{C}$ is a semisimple $*$-category. Consider the $*$-algebra $A=\oplus_{i \in I_{\mathcal{C}}} \mathbb{C}$. Then Proposition 5.3 provides an equivalence $F: \mathcal{C} \rightarrow \operatorname{Rep}_{f} A$ of categories such that $F\left(X_{i}\right) \cong p_{i}$ for all $i \in I_{\mathcal{C}}$. Let $K: \operatorname{Rep}_{f} A \rightarrow \mathcal{H}$ be the forgetful functor. Then $K \circ F$ is an embedding functor for $\mathcal{C}$ as a $*$-category. By Proposition 5.4 and Example 5.5 we also conclude that

$$
\operatorname{Nat}(K \circ F) \cong \operatorname{Nat} I d \cong \prod_{i \in I_{\mathcal{C}}} \mathbb{C}
$$

(Here of course, it does not make sense to say that the functors $I d$ and $K \circ F$ are unitarily equivalent.) Suppose now that $\mathcal{C}$ is in addition a tensor category. Then the faithful functor $K \circ F$ cannot in general be monoidal. To see this let $X_{i}, X_{j}$ with $i, j \in I_{\mathcal{C}}$. Then $K \circ F\left(X_{i}\right) \cong K\left(p_{i}\right) \cong \mathbb{C}$, so $K \circ F\left(X_{i}\right) \otimes K \circ F\left(X_{j}\right) \cong \mathbb{C}$, whereas

$$
K \circ F\left(X_{i} \otimes X_{j}\right) \cong \bigoplus_{k \in I_{\mathcal{C}}} N_{i j}^{k} K \circ F\left(X_{k}\right) \cong \bigoplus_{k \in I_{\mathcal{C}}} N_{i j}^{k} \mathbb{C},
$$

where we used the decomposition $X_{i} \otimes X_{j} \cong \oplus_{k \in I_{\mathcal{C}}} N_{i j}^{k} X_{k}$. 
Given a *-preserving functor $E: \mathcal{C} \rightarrow \mathcal{K}$ and writing $B=$ Nat $E$ it is natural to ask under which conditions the functor $F: \mathcal{C} \rightarrow \operatorname{Rep}_{\mathcal{K}} B$ defined in Proposition 5.3 is an equivalence of categories. Instead of pursuing this line further we restrict ourselves to the case where $\mathcal{C}$ is semisimple and $\mathcal{K}=\mathcal{H}$. Then End $E(X)$ is a finite dimensional matrix algebra for every $X \in \mathcal{C}$ and Proposition 5.4 shows that Nat $E$ is a direct product of matrix algebras. The representation theory of such algebras is quite intricate, cf. [13]. There it is shown, among other results, that every irreducible representation of $B=\prod_{i \in I} B\left(H_{i}\right)$ is equivalent to a projection $p_{i}$ iff the index set $I$ has less than measurable cardinality. In practice, this is no serious restriction but for reasons which will become clear in the next subsection we prefer to work with direct sums instead of direct products.

TheOREM 5.8 Let $\mathcal{C}$ be a semisimple $*$-category and $E: \mathcal{C} \rightarrow \mathcal{H}$ a $*$-preserving functor. The $*$-algebra $A=$ $\oplus_{i \in I_{\mathcal{C}}} B\left(E\left(X_{i}\right)\right)$ embeds canonically into Nat $E \cong \prod_{i \in I_{\mathcal{C}}} B\left(E\left(X_{i}\right)\right)$. The functor

$$
F: \mathcal{C} \rightarrow \operatorname{Rep}_{f} A, \quad F(X)=\left(E(X), \pi_{X}\right), \pi_{X}(a)=a_{X} \forall a \in A, \quad F(s)=E(s)
$$

satisfies $K \circ F=E$. It gives rise to an equivalence of categories iff $E$ is faithful.

Proof. $F$ as defined above differs from the functor defined in Proposition 5.3 only in the restriction of the representations $\pi_{X}$ to $A \subset$ Nat $E$. Thus it clearly is a $*$-preserving functor. By definition it is clear that $K \circ F=E$. Since $F$ coincides with $E$ on morphisms, $E$ is faithful if $F$ is an equivalence. Assume now that $E$ is faithful. Let $X \in \mathcal{C}$ be irreducible. Then $F(X)$ is a representation $\pi_{X}: a \mapsto a_{X}$ of $A$ on the Hilbert space $E(X)$. As is clear from the proof of Proposition 5.4, for $X=X_{i}$ we have $\pi_{X}=p_{i}$, which clearly is irreducible. Since $a \in A$ is a natural transformation, $\pi_{X}: a \mapsto a_{X}$ is an irreducible $*$-representation whenever $X$ is irreducible. Consider $i \neq j, i, j \in I_{\mathcal{C}}$. By Proposition 2.10, the projections $p_{i}=F\left(X_{i}\right), p_{j}=F\left(X_{j}\right)$ are inequivalent $*$-representations. By semisimplicity of $\operatorname{Rep}_{f} A$ this implies that $F$ is full. Since the $p_{i}, i \in I_{\mathcal{C}}$ exhaust the equivalence classes of irreducible $*$-representations of $A$ it is clear that $F$ is essentially surjective, completing the proof.

REMARK 5.9 The theorem remains true if one replaces $A$ by the direct product algebra Nat $E$ provided one makes the additional assumption that the set $I_{\mathcal{C}}$ of isomorphism classes of $\mathcal{C}$ has less than measurable cardinality.

\subsection{Concrete Tensor *-Categories and Discrete Quantum Semigroups}

From now on, $\mathcal{C}$ will be a semisimple strict tensor $*$-category. (As discussed in the Introduction, the strictness assumption does not entail a loss of generality.) We will introduce other requirements as we proceed. By Theorem 5.8 we have associated a $*$-algebra $A$ to a $*$-preserving functor $E: \mathcal{C} \rightarrow \mathcal{H}$. The aim of this subsection is to endow $A$ with the structure of a discrete aqg under suitable conditions on $\mathcal{C}$ and $E$. To bring out the special rôle of the target category $\mathcal{H}$, we work in a more general setting as far as seems justifiable.

Let $E: \mathcal{C} \rightarrow \mathcal{D}$ be a $*$-preserving tensor functor into some tensor $*$-category $\mathcal{D}$. Recall that by Proposition 5.1, Nat $E$ is a unital $*$-algebra for every $*$-preserving functor $E: \mathcal{C} \rightarrow \mathcal{D}$.

Proposition 5.10 Let $\mathcal{C}, \mathcal{D}$ be tensor $*$-categories, $\mathcal{C}$ being strict, and let $E: \mathcal{C} \rightarrow \mathcal{D}$ be a $*$-preserving tensor functor. Then

$$
\tilde{\Delta}: \operatorname{Nat} E \rightarrow \operatorname{Nat} E \otimes, \quad b=\left(b_{X}\right) \mapsto \tilde{\Delta}(b)=\left(\tilde{\Delta}(b)_{Y, Z}\right), \quad \tilde{\Delta}(b)_{Y, Z}=b_{Y \otimes Z}
$$

defines a unital $*$-homomorphism.

Proof. The morphisms in $\mathcal{C} \times \mathcal{C}$ are of the form $s \times t$ where $s: Y \rightarrow Y^{\prime}, t: Z \rightarrow Z^{\prime}$. Then $s \otimes t \in$ $\operatorname{Mor}\left(Y \otimes Z, Y^{\prime} \otimes Z^{\prime}\right)$. We compute

$$
(E \otimes)(s \times t) \circ \tilde{\Delta}(b)_{Y, Z}=E(s \otimes t) \circ b_{Y \otimes Z}=b_{Y^{\prime} \otimes Z^{\prime}} \circ E(s \otimes t)=\tilde{\Delta}(b)_{Y^{\prime}, Z^{\prime}} \circ(E \otimes)(s \times t),
$$


where we used $b=\left(b_{X}\right) \in \operatorname{Nat} E$. We conclude that $\tilde{\Delta}(b) \in \operatorname{Nat} E \otimes$. That $b \mapsto \tilde{\Delta}(b)$ is a unital $*$-homomorphism follows immediately from Proposition 5.1.

We now consider the analogues of $(\Delta \otimes \iota), \mathcal{C}$ being strict, and $(\iota \otimes \Delta)$ in the present general setting. $\otimes^{2}$ will denote the functor $\otimes \circ(\otimes \times I d)=\otimes \circ(I d \times \otimes): \mathcal{C} \times \mathcal{C} \times \mathcal{C} \rightarrow \mathcal{C}$, where we have used the strictness of $\mathcal{C}$.

Proposition 5.11 Let $\mathcal{C}, \mathcal{D}$ be tensor $*$-categories and let $E: \mathcal{C} \rightarrow \mathcal{D}$ be a $*$-preserving tensor functor. There are unital $*$-homomorphisms $\widetilde{\Delta \otimes \iota, \iota \otimes \Delta}:$ Nat $E \otimes \rightarrow$ Nat $E \otimes^{2}$ defined by

$\widetilde{\Delta \otimes \iota}: \quad$ Nat $E \otimes \rightarrow$ Nat $E \otimes^{2}, \quad b=\left(b_{X, Y}\right) \mapsto(\widetilde{\Delta \otimes \iota})(b)=\left(\widetilde{(\widetilde{\Delta} \iota)}(b)_{U, V, W}\right), \quad(\widetilde{\Delta \otimes \iota})(b)_{U, V, W}=b_{U \otimes V, W}$,

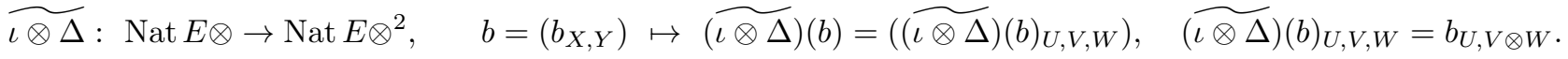

These morphisms satisfy $(\widetilde{\Delta \otimes \iota}) \tilde{\Delta}=(\widetilde{\iota \otimes \Delta}) \tilde{\Delta}$.

Proof. That $(\widetilde{\Delta \otimes \iota})(b) \in$ Nat $E \otimes^{2}$ for $b \in$ Nat $E \otimes$ follows from the following computation:

$$
\begin{aligned}
& \left(E \otimes^{2}\right)(s \times t \times u) \circ(\widetilde{\Delta \otimes \iota})(b)_{Y, Z, W}=E(s \otimes t \otimes u) \circ b_{Y \otimes Z, W} \\
& =b_{Y^{\prime} \otimes Z^{\prime}, W^{\prime}} \circ E(s \otimes t \otimes u)=(\widetilde{\Delta \otimes \iota})(b)_{Y^{\prime}, Z^{\prime}, W^{\prime}} \circ\left(E \otimes^{2}\right)(s \times t \times u),
\end{aligned}
$$

whenever $s: U \rightarrow U^{\prime}, t: V \rightarrow V^{\prime}, u: W \rightarrow W^{\prime}$. The argument for $\left.\widetilde{(\iota \otimes}\right)(b)$ is completely analogous. Clearly, both maps $(\widetilde{\Delta \otimes \iota}),(\widetilde{\iota \otimes \Delta})$ are unital $*$-homomorphisms. The coassociativity property follows from

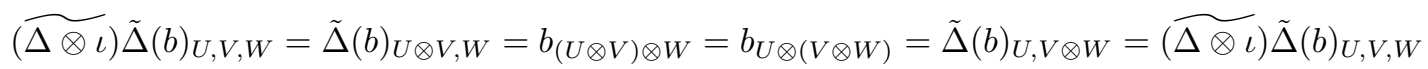

with $b \in$ End $E$, where we have used the strictness of $\mathcal{C}$.

Proposition 5.12 There are unital $*$-homomorphisms $\tilde{\varepsilon}: \operatorname{Nat} E \rightarrow \operatorname{End}(E(\mathbf{1}))$ and $\widetilde{\varepsilon \otimes} \iota, \widetilde{\otimes} \varepsilon:$ Nat $E \otimes \rightarrow$ Nat $E$ defined by

$$
\begin{aligned}
& \tilde{\varepsilon}: \quad \quad \quad \operatorname{Nat} E \rightarrow \operatorname{End}(E(\mathbf{1})), \quad b=\left(b_{X}\right) \mapsto b_{\mathbf{1}}, \\
& \widetilde{\varepsilon \otimes \iota}: \quad \text { Nat } E \otimes \rightarrow \text { Nat } E, \quad b=\left(b_{X, Y}\right) \mapsto(\widetilde{\varepsilon \otimes} \iota)(b)=\left((\widetilde{\varepsilon \otimes} \iota)(b)_{X}\right), \quad(\widetilde{\varepsilon \otimes} \iota)(b)_{X}=b_{1, X}, \\
& \widetilde{\iota \otimes \varepsilon}: \quad \text { Nat } E \otimes \rightarrow \operatorname{Nat} E, \quad b=\left(b_{X, Y}\right) \mapsto(\widetilde{\iota \otimes \varepsilon})(b)=\left((\widetilde{\iota \otimes \varepsilon})(b)_{X}\right), \quad(\widetilde{\iota \otimes \varepsilon})(b)_{X}=b_{X, \mathbf{1}} \text {. }
\end{aligned}
$$

They satisfy $(\widetilde{\varepsilon \otimes} \iota) \tilde{\Delta}=(\widetilde{\iota \otimes}) \tilde{\Delta}=\iota$.

Proof. Clearly, the map $\tilde{\varepsilon}: b=\left(b_{X}\right) \mapsto b_{1} \in \operatorname{End} E(\mathbf{1})$ is a unital $*$-homomorphism. The proofs that $(\widetilde{\varepsilon \otimes \iota})(b),(\widetilde{\iota \otimes \varepsilon})(b)$ are in fact in Nat $E$ and that the maps $b \mapsto(\widetilde{\varepsilon \otimes \iota})(b), b \mapsto(\widetilde{\iota \otimes \varepsilon})(b)$ are $*$-homomorphisms proceed along the same lines as for coproducts and are therefore omitted. For $b \in$ Nat $E$ the computation

$$
(\widetilde{\varepsilon \otimes \iota}) \tilde{\Delta}(b)=\tilde{\Delta}(b)_{\mathbf{1}, X}=b_{\mathbf{1} \otimes X}=b_{X}=b_{X \otimes \mathbf{1}}=\tilde{\Delta}(b)_{X, \mathbf{1}}=(\widetilde{\iota \otimes \varepsilon}) \tilde{\Delta}(b),
$$

where we used the strictness of the unit $\mathbf{1}$, concludes the proof.

We have thus seen that we can define a 'coproduct' $\tilde{\Delta}:$ Nat $E \rightarrow$ Nat $E \otimes$ and a 'counit' $\tilde{\varepsilon}:$ Nat $E \rightarrow \operatorname{End} E(\mathbf{1})$ satisfying analogues of the usual properties. A priori, however, Nat $E \otimes$ has nothing to do with Nat $E \otimes$ Nat $E$, regardless of how we interpret the tensor product. The following result indicates how to proceed. We now assume that $\mathcal{C}$ is semisimple. As before, by $I_{\mathcal{C}}$ we denote the set of isomorphism classes of irreducible objects of $\mathcal{C}$. For every $i \in I_{\mathcal{C}}$ we pick a representative $X_{i}$.

DefinITION 5.13 Given a tensor $*$-category $\mathcal{C}$, an embedding functor (for $\mathcal{C}$ ) is a faithful $*$-preserving tensor functor $E: \mathcal{C} \rightarrow \mathcal{H}$. A concrete tensor $*$-category is a tensor $*$-category together with an embedding functor. 
Proposition 5.14 Let $\mathcal{C}$ be a semisimple tensor $*$-category and $E: \mathcal{C} \rightarrow \mathcal{H}$ an embedding functor. With the *-algebra $A=\bigoplus_{i \in I_{\mathcal{C}}} B\left(E\left(X_{i}\right)\right)$ we have the following canonical *-isomorphisms:

$$
\begin{array}{lll}
\text { Nat } E \stackrel{\psi_{1}}{\longrightarrow} M(A) & =\prod_{i \in I_{\mathcal{C}}} B\left(E\left(X_{i}\right)\right), \\
\text { Nat } E \otimes & \stackrel{\psi_{2}}{\longrightarrow} M(A \otimes A) & =\prod_{i, j \in I_{\mathcal{C}}} B\left(E\left(X_{i}\right)\right) \otimes B\left(E\left(X_{j}\right)\right), \\
\text { Nat } E \otimes^{2} \stackrel{\psi_{3}}{\longrightarrow} M(A \otimes A \otimes A) & =\prod_{i, j, k \in I_{\mathcal{C}}} B\left(E\left(X_{i}\right)\right) \otimes B\left(E\left(X_{j}\right)\right) \otimes B\left(E\left(X_{k}\right)\right) .
\end{array}
$$

Proof. The isomorphism $\psi_{1}$ was established in Propositions 5.4 and 2.10 and suppressed subsequently. As to $\psi_{2}$, we have

$$
\begin{aligned}
\text { Nat } E \otimes & =\prod_{i, j \in I_{\mathcal{C}}} \text { End } E\left(X_{i} \otimes X_{j}\right) \cong \prod_{i, j \in I_{\mathcal{C}}} \text { End }\left(E\left(X_{i}\right) \otimes E\left(X_{j}\right)\right) \\
& =\prod_{i, j \in I_{\mathcal{C}}} B\left(E\left(X_{i}\right) \otimes E\left(X_{j}\right)\right)=\prod_{i, j \in I_{\mathcal{C}}} B\left(E\left(X_{i}\right)\right) \otimes B\left(E\left(X_{j}\right)\right)=M(A \otimes A) .
\end{aligned}
$$

Here we used the identification $B(H \otimes K) \equiv B(H) \otimes B(K)$ for finite dimensional Hilbert spaces and the identifications made after Propositions 2.10 and 5.4. The isomorphism between End $E\left(X_{i} \otimes X_{j}\right)$ and End $E\left(X_{i}\right) \otimes$ $E\left(X_{j}\right)$ is induced in the canonical way from the isomorphism $d_{X_{i}, X_{j}}^{E}: E\left(X_{i}\right) \otimes E\left(X_{j}\right) \rightarrow E\left(X_{i} \otimes X_{j}\right)$ which makes $E$ monoidal. Unitarity of the $d_{X_{i}, X_{j}}^{E}$ implies that $\psi_{2}$ is $*$-preserving. The argument for $\psi_{3}$ is completely analogous.

REMARK 5.15 In the above proof it was crucial that the canonical $*$-homomorphisms $\otimes:$ End $X \otimes_{\mathbb{C}}$ End $Y \rightarrow$ $\operatorname{End}(X \otimes Y)$ are $*$-isomorphisms for all $X, Y \in \mathcal{H}$. A semisimple tensor $*$-category has this property iff every object is a finite direct sum of copies of the tensor unit 1. [Proof. The 'if' clause is easy. As to the 'only if' part, let $Z$ be irreducible and consider $X \cong Y \cong \mathbf{1} \oplus Z$, thus $X \otimes Y \cong \mathbf{1} \oplus Z \oplus Z \oplus Z^{2}$. If $Z \neq \mathbf{1}$ then End $X \otimes_{\mathbb{C}}$ End $Y \cong \mathbb{C}^{2} \otimes \mathbb{C}^{2}$ is commutative whereas $\operatorname{End}(X \otimes Y)$ is non-commutative since $X \otimes Y$ contains the irreducible $Z$ with multiplicity at least two. This contradicts End $X \otimes_{\mathbb{C}} \operatorname{End} Y \cong \operatorname{End}(X \otimes Y)$.] This property is known to characterize the (semisimple) tensor $*$-category $\mathcal{H}$ uniquely up to equivalence. We thus see that with $E=I d: \mathcal{C} \rightarrow \mathcal{C}$, we cannot conclude that Nat $E \otimes \cong M(A \otimes A)$. This is consistent with the computation of Nat $\otimes$ in Example 5.6.

From now on we restrict ourselves to the case we are ultimately interested in.

Definition 5.16 Let $\mathcal{C}$ be a semisimple tensor $*$-category and let $E: \mathcal{C} \rightarrow \mathcal{H}$ be an embedding functor. Using the preceding results we define unital $*$-homomorphisms as follows.

$$
\begin{array}{lll}
\bar{\varepsilon}: M(A) \rightarrow \mathbb{C}, & a \mapsto \tilde{\varepsilon} \circ \psi_{1}^{-1}(a), \\
\bar{\Delta}: M(A) \rightarrow M(A \otimes A), & a \mapsto \psi_{2} \circ \tilde{\Delta} \circ \psi_{1}^{-1}(a) .
\end{array}
$$

Here we have implicitly used the irreducibility of $E\left(\mathbf{1}_{\mathcal{C}}\right) \cong \mathbf{1}_{\mathcal{H}} \cong \mathbb{C}$, giving rise to the isomorphism $\psi_{0}$ : End $E(\mathbf{1}) \rightarrow \mathbb{C}, c \operatorname{id}_{E(\mathbf{1})} \mapsto c$.

REMARK 5.17 1. In the sequel we will usually use the isomorphisms $\psi_{1}, \psi_{2}$ to identify $M(A) \equiv \operatorname{Nat} E, M(A \otimes$ $A) \equiv$ Nat $E \otimes$ and suppress the symbols $\psi_{1}, \psi_{2}$. Thus for $a \in M(A), i \in I, a_{i}$ will denote both the $i$-component of $a$ in $\prod_{i \in I} B\left(E\left(X_{i}\right)\right)$ and the action $a_{X_{i}}$ on $E\left(X_{i}\right)$ of the natural transformation $\psi_{1}^{-1}(a) \in$ Nat $E$.

2. Let $a \in M(A)$. As $\Delta(a)_{i j}=a_{X_{i} \otimes X_{j}}$ and $(a \otimes a)_{i j}=a_{i} \otimes a_{j}$ we find

$$
\Delta(a)=a \otimes a \quad \Leftrightarrow \quad a_{X_{i} \otimes X_{j}}=a_{X_{i}} \otimes a_{X_{j}} \quad \forall i, j \in I .
$$

Thus the grouplike elements of $M(A)$ are in one-to-one correspondence with those natural transformations of the embedding functor which respect the tensor structure. 
Lemma 5.18 The restriction $\Delta=\bar{\Delta} \uparrow A$ is a non-degenerate $*$-homomorphism from $A$ to $M(A \otimes A)$, i.e. $\Delta(A)(A \otimes A)=(A \otimes A) \Delta(A)=(A \otimes A)$.

Proof. To simplify the notation we write $H_{i}=B\left(E\left(X_{i}\right)\right)$ and use the isomorphisms $\psi_{1}, \psi_{2}$ to identify $M(A)$ with Nat $E$ and $M(A \otimes A)$ with Nat $E \otimes$. We will only prove $\Delta(A)(A \otimes A)=(A \otimes A)$, the proof of the other identity being completely analogous. As $A=\oplus_{i} B\left(H_{i}\right)$, it suffices to show that for every $i, j \in I_{\mathcal{C}}$ there is a $c \in A$ with $\Delta(c)\left(I_{i} \otimes I_{j}\right)=I_{i} \otimes I_{j}$, since this implies $a_{i} \otimes b_{j}=\Delta(c)\left(I_{i} \otimes I_{j}\right)\left(a_{i} \otimes b_{j}\right) \in \Delta(A)(A \otimes A)$ for all $a_{i} \in B\left(H_{i}\right), b_{j} \in B\left(H_{j}\right)$. Recall from the Propositions 5.14 and 5.4 that $M(A)$ is isomorphic to the *algebra of natural transformations of $E$, and these are uniquely determined by their actions on the irreducibles: $M(A) \ni b \leftrightarrow\left(b_{i}\right)$, where $b_{i} \equiv b_{X_{i}}$. Similarly, $M(A \otimes A) \ni b \leftrightarrow\left(b_{i j}\right)$ with $b_{i j} \equiv b_{X_{i}, X_{j}}$. By the definition of $\tilde{\Delta}$ we have $\tilde{\Delta}(b)_{i j}=b_{X_{i} \otimes X_{j}}$. As usual, let $\left(v_{i j}^{k \alpha}, \alpha=1, \ldots, N_{i j}^{k}\right)$ be an orthonormal basis in $\operatorname{Mor}\left(X_{k}, X_{i} \otimes X_{j}\right)$ for all $i, j, k \in I_{\mathcal{C}}$. Since $b \in M(A) \equiv$ End $E$ is a natural transformation we have

$$
\tilde{\Delta}(b)_{i j}=b_{X_{i} \otimes X_{j}}=b_{X_{i} \otimes X_{j}} \circ \operatorname{id}_{E\left(X_{i} \otimes X_{j}\right)}=b_{X_{i} \otimes X_{j}} \circ E\left(\sum_{k \alpha} v_{i j}^{k \alpha} \circ v_{i j}^{k \alpha *}\right)=\sum_{k \alpha} E\left(v_{i j}^{k \alpha}\right) \circ b_{k} \circ E\left(v_{i j}^{k \alpha *}\right) .
$$

Now fix $i_{0}, j_{0} \in I_{\mathcal{C}}$ and let $b_{k}=\operatorname{id}_{E\left(X_{k}\right)}$ if $X_{k}$ is contained in $X_{i_{0}} \otimes X_{j_{0}}$ and $b_{k}=0$ otherwise. Since $X_{k} \prec X_{i_{0}} \otimes X_{j_{0}}$ only for finitely many $k$ we have $b=\left(b_{k}\right) \in A$ and

$$
\left.\tilde{\Delta}(b)_{i_{0} j_{0}}=\sum_{k \alpha} E\left(v_{i_{0} j_{0}}^{k \alpha}\right) \circ \operatorname{id}_{E\left(X_{k}\right)} \circ E\left(v_{i_{0} j_{0}}^{k \alpha *}\right)=\sum_{k \alpha} E\left(v_{i_{0} j_{0}}^{k \alpha} \circ v_{i_{0} j_{0}}^{k \alpha *}\right)=E\left(\operatorname{id}_{X_{i_{0}} \otimes X_{j_{0}}}\right)=\operatorname{id}_{E\left(X_{i_{0}} \otimes X_{j_{0}}\right.}\right) .
$$

Having identified Nat $E \otimes \equiv M(A \otimes A)$ this precisely means that the $\left(i_{0}, j_{0}\right)$-component of $\Delta(b) \in M(A \otimes A)=$ $\prod_{i j} B\left(H_{i}\right) \otimes B\left(H_{j}\right)$ is the identity $I_{i_{0}} \otimes I_{j_{0}}$ and therefore $\Delta(b)\left(I_{i_{0}} \otimes I_{j_{0}}\right)=\left(I_{i_{0}} \otimes I_{j_{0}}\right)$ as desired.

Corollary 5.19 Defining $*$-homomorphisms $\varepsilon: A \rightarrow \mathbb{C}$ and $\Delta: A \rightarrow M(A \otimes A)$ by $\varepsilon=\bar{\varepsilon} \uparrow A$ and $\Delta=\bar{\Delta} \uparrow A$, $\bar{\varepsilon}$ and $\bar{\Delta}$ are the unique extensions of $\varepsilon$ and $\Delta$, respectively, to $M(A)$.

Proof. It is clear that $\varepsilon$ is not identically zero, thus non-degenerate. Non-degeneracy of $\Delta$ has been proven in Lemma 5.18. Now the claim follows from the fact [35] that a non-degenerate $*$-homomorphism $\phi: A \rightarrow M(B)$ has a unique extension to a unital $*$-homomorphism $\phi: M(A) \rightarrow M(B)$.

Proposition 5.20 Let $\mathcal{C}$ be a semisimple tensor $*$-category and $E: \mathcal{C} \rightarrow \mathcal{H}$ an embedding functor. Then the *-algebra $A=\oplus_{i \in I_{\mathcal{C}}} B\left(E\left(X_{i}\right)\right)$ is a discrete quantum semigroup, i.e. has a non-degenerate coproduct $\Delta: A \rightarrow$ $M(A \otimes A)$ in the sense of [35] and a counit $\varepsilon: A \rightarrow \mathbb{C}$.

Proof. By Lemma 5.18, $\Delta$ is non-degenerate, thus also $\Delta \otimes \iota: A \otimes A \rightarrow M(A \otimes A) \otimes A \subset M(A \otimes A \otimes A)$ is non-degenerate and extends uniquely to $\overline{\Delta \otimes \iota}: M(A \otimes A) \rightarrow M(A \otimes A \otimes A)$. Another unital $*$-homomorphism $M(A \otimes A) \rightarrow M(A \otimes A \otimes A)$ is given by $\psi_{3} \circ\left(\widetilde{\Delta \otimes \iota)} \circ \psi_{2}^{-1}\right.$. The latter clearly restricts to $\Delta \otimes \iota$ on $A \otimes A$, thus it coincides with $\overline{\Delta \otimes \iota}$ on all of $M(A \otimes A)$. In the same way we make sense of $\overline{\iota \otimes \Delta}: M(A \otimes A) \rightarrow M(A \otimes A \otimes A)$ and see that it coincides with $\left.\psi_{3} \circ \widetilde{(\iota \otimes \Delta}\right) \circ \psi_{2}^{-1}$. Now, the coassociativity property proved in Proposition 5.11 implies

$$
\overline{\Delta \otimes \iota} \circ \bar{\Delta}=\overline{\iota \otimes \Delta} \circ \bar{\Delta}: M(A) \rightarrow M(A \otimes A \otimes A),
$$

which is the proper formulation of coassociativity for $(A, \Delta)$.

As observed earlier, the $*$-homomorphism $\varepsilon: A \rightarrow \mathbb{C}$ is non-degenerate, thus $\varepsilon \otimes \iota, \iota \otimes \varepsilon: A \otimes A \rightarrow A$ are non-degenerate too and have unique extensions $\overline{\varepsilon \otimes \iota}, \overline{\iota \otimes \varepsilon}$ to $M(A \otimes A)$. Again, it is clear that $\overline{\varepsilon \otimes \iota}=$ $\psi_{1} \circ(\widetilde{\varepsilon \otimes \iota}) \circ \psi_{2}^{-1}$ and similarly for $\overline{\iota \otimes \varepsilon}$. Thus with Proposition 5.12 we conclude

$$
\overline{\varepsilon \otimes \iota} \circ \bar{\Delta}=\overline{\iota \otimes \varepsilon} \circ \bar{\Delta}=\mathrm{id}: M(A) \rightarrow M(A) .
$$

We conclude that $(A, \Delta)$ is a discrete quantum semigroup. 
TheOREm 5.21 Let $\mathcal{C}$ be a semisimple tensor $*$-category and $E: \mathcal{C} \rightarrow \mathcal{H}$ an embedding functor. Let $(A, \Delta)$ be the corresponding discrete quantum semigroup. Then the functor $F: \mathcal{C} \rightarrow \operatorname{Rep}_{f}(A, \Delta)$ defined by

$$
F(X)=\left(E(X), \pi_{X}\right), \quad \pi_{X}(a)=a_{X} \forall a \in A, \quad F(s)=E(s)
$$

provides an equivalence of tensor $*$-categories such that $K \circ F=E$.

Proof. With $A$ and $F: \mathcal{C} \rightarrow \operatorname{Rep}_{f} A$ as defined in Theorem 5.8 we have an equivalence of categories. It remains to show that $F: \mathcal{C} \rightarrow \operatorname{Rep}_{f}(A, \Delta)$ is monoidal for the monoidal structure of $\operatorname{Rep}_{f}(A, \Delta)$ in Definition 3.12. Thus we must exhibit isomorphisms $d_{X, Y}^{F}: F(X) \otimes F(Y) \rightarrow F(X \otimes Y)$ satisfying the conditions of Definition 2.1. In view of $F(X)=\left(E(X), \pi_{X}\right)$, an obvious candidate is the isomorphism $d_{X, Y}^{E}: E(X) \otimes E(Y) \rightarrow E(X \otimes Y)$ which we have by virtue of the assumed monoidality of $E$. If we set $d_{X, Y}^{F}=d_{X, Y}^{E}$ for all $X, Y \in \mathcal{C}$, the equations in Definition 2.1 are clearly satisfied. It only remains to prove that $d_{X, Y}^{F}$ is in $\operatorname{Mor}_{\operatorname{Rep}_{f}(A, \Delta)}(F(X) \otimes F(Y), F(X \otimes Y))$, i.e.

$$
d_{X, Y}^{F} \circ\left(\pi_{X} \times \pi_{Y}\right)(a)=\pi_{X \otimes Y}(a) \circ d_{X, Y}^{F} \quad \forall a \in A .
$$

Keeping in mind the various identifications we have made so far, we get

$$
\begin{gathered}
d_{X, Y}^{F} \circ\left(\pi_{X} \times \pi_{Y}\right)(a)=d_{X, Y}^{F} \circ \overline{\left(\pi_{X} \otimes \pi_{Y}\right)} \Delta(a)=d_{X, Y}^{F} \circ \overline{\left(\pi_{X} \otimes \pi_{Y}\right) \bar{\Delta}}(a) \\
=d_{X, Y}^{F} \circ \overline{\left(\pi_{X} \otimes \pi_{Y}\right)} \psi_{2} \tilde{\Delta}(a)=\pi_{X, Y} \tilde{\Delta}(a) \circ d_{X, Y}^{F}=a_{X \otimes Y} \circ d_{X, Y}^{F}=\pi_{X \otimes Y}(a) \circ d_{X, Y}^{F}
\end{gathered}
$$

for all $a \in A$. (The reader is invited to check the required identity explicitly for $X=X_{i}, Y=X_{j}$, as suffices by naturality of the isomorphisms $d^{F}$.)

\subsection{Conjugates and Antipodes: Discrete Quantum Groups}

We have already seen that the concrete semisimple tensor $*$-categories $\operatorname{Rep}_{f}(A, \Delta)$ and $\operatorname{Corep}_{f}(\hat{A}, \hat{\Delta})$ come with conjugates whenever $(A, \Delta)$ is a discrete aqg. In this section we prove the converse. We start off with a couple of preparatory results.

LEMMA 5.22 Let $\mathcal{C}$ be a semisimple tensor $*$-category with conjugates and let $E: \mathcal{C} \rightarrow \mathcal{H}$ be an embedding functor. Then the coproduct $\Delta: A \rightarrow M(A \otimes A)$ for the discrete quantum semigroup $(A, \Delta)$ given by Proposition 5.20 has the following inclusion properties

$$
(1 \otimes A) \Delta(A) \subset A \otimes A, \quad(A \otimes 1) \Delta(A) \subset A \otimes A .
$$

Proof. We prove only the inclusion $(A \otimes 1) \Delta(A) \subset A \otimes A$, the proof of the inclusion $(1 \otimes A) \Delta(A) \subset A \otimes A$ being completely analogous. We need to show that $(a \otimes 1) \Delta(b) \in A \otimes A$ for all $a, b \in A$. It is clearly sufficient to show this for $b=\left(b_{i}\right)$ where all components except $i=m$ vanish and for $a=I_{n}$. By the calculation done in the proof of Lemma 5.18 we have

$$
\Delta(b)_{i j}=b_{X_{i} \otimes X_{j}}=\sum_{k \in I_{\mathcal{C}}} \sum_{\alpha=1}^{N_{i j}^{k}} E\left(V_{i j}^{k \alpha}\right) \circ b_{k} \circ E\left(V_{i j}^{k \alpha *}\right) \in \operatorname{End} E\left(X_{i} \otimes X_{j}\right) .
$$

In view of $a=I_{n}$ and $b_{i}=0$ for $i \neq m$ we obtain

$$
((a \otimes 1) \Delta(b))_{i j}=\delta_{i, n} \sum_{\alpha=1}^{N_{n j}^{m}} E\left(V_{n j}^{m \alpha}\right) \circ b_{m} \circ E\left(V_{n j}^{m \alpha *}\right) .
$$

Now, since $\mathcal{C}$ has conjugates we have isomorphisms $\operatorname{Mor}\left(X_{n} \otimes X_{j}, X_{m}\right) \cong \operatorname{Mor}\left(X_{j}, \overline{X_{n}} \otimes X_{m}\right)$. By semisimplicity, for fixed $n, m$ the latter space is non-zero only for finitely many $j \in I_{\mathcal{C}}$. Thus $((a \otimes 1) \Delta(b))_{i j}$ is non-zero only for $i=n$ and finitely many $j$ and therefore $(a \otimes 1) \Delta(b) \in A \otimes A$. 
Proposition 5.23 Let $\mathcal{C}$ be a semisimple tensor $*$-category with conjugates and let $E: \mathcal{C} \rightarrow \mathcal{H}$ be an embedding functor. Let $b=\left(b_{X, Y}\right) \in$ Nat $E \otimes$. Using the identifications $B((E(X \otimes Y))=B(E(X)) \otimes B(E(Y))$, for any objects $X$ and $Y$ in $\mathcal{C}$, we may write $b_{X, X}=\sum_{k} a_{X}^{k} \otimes b_{X}^{k} \in B(E(X)) \otimes B(E(X))$, and then define $\tilde{m}(b)_{X} \in B(E(X))$ by

$$
\tilde{m}(b)_{X}=\sum_{k} a_{X}^{k} \circ b_{X}^{k}
$$

for any object $X \in \mathcal{C}$. This gives a unital linear map $\tilde{m}:$ Nat $E \otimes \rightarrow$ Nat $E$ which sends $b \in N a t E \otimes$ to $\tilde{m}(b)$. Furthermore, it restricts to the linearized multiplication $m: A \otimes A \rightarrow A$ on $A \subset$ Nat $E$.

Proof. The bilinear multiplication map from $B(E(X)) \otimes B(E(X))$ to $B(E(X))$ exists since $E(X)$ is finite dimensional. The rest is obvious.

Proposition 5.24 Let $\mathcal{C}$ be a semisimple tensor $*$-category with conjugates and let $E: \mathcal{C} \rightarrow \mathcal{H}$ be an embedding functor. Then the discrete quantum semigroup $(A, \Delta)$ given by Proposition 5.20 is a discrete aqg. The coinverse $S: A \rightarrow A$ is given by

$$
S(a)_{X}=E\left(\operatorname{id}_{X} \otimes r_{X}^{*}\right) \circ \operatorname{id}_{E(X)} \otimes a_{\bar{X}} \otimes \operatorname{id}_{E(X)} \circ E\left(\bar{r}_{X} \otimes \operatorname{id}_{X}\right)
$$

for all $a \in A \subset$ Nat $E$ and objects $X$ in $\mathcal{C}$, and where $\left(r_{X}, \bar{r}_{X}, \bar{X}\right)$ is any solution of the conjugate equations associated to $X$.

Proof. In view of Proposition 2.4 it is sufficient to show that $(A \otimes 1) \Delta(A) \subset A \otimes A$ and $(1 \otimes A) \Delta(A) \subset A \otimes A$ and to produce an invertible coinverse $S$. We thus get an aqg since Theorem 2.13 provides a (non-zero) leftinvariant positive functional for discrete multiplier Hopf $*$-algebras. Now the inclusions $(A \otimes 1) \Delta(A) \subset A \otimes A$ and $(1 \otimes A) \Delta(A) \subset A \otimes A$ are the content of Lemma 5.22.

We show that the map $S: A \rightarrow A$ given by the formula in the theorem is well-defined. We first show that for $a \in A$ and $X \in \mathcal{C}$, the formula for $S(a)_{X} \in$ End $E(X)$ does not depend on the choice of solution $\left(r_{X}, \bar{r}_{X}, \bar{X}\right)$ of the conjugate equations associated to $X$. Suppose therefore that $\left(r_{X}^{\prime}, \bar{r}_{X}^{\prime}, \bar{X}^{\prime}\right)$ is another solution of the conjugate equations associated to $X$. By [23] there exists a unique invertible $t \in \operatorname{Hom}\left(\bar{X}, \bar{X}^{\prime}\right)$ such that $r_{X}^{\prime}=t \otimes \operatorname{id}_{X} \circ r_{X}$ and $\bar{r}_{X}^{\prime}=\operatorname{id}_{X} \otimes t^{*-1} \circ \bar{r}_{X}$. Thus

$$
\begin{aligned}
& E\left(\operatorname{id}_{X} \otimes r_{X}^{\prime *}\right) \circ \operatorname{id}_{E(X)} \otimes a_{\bar{X}^{\prime}} \otimes \operatorname{id}_{E(X)} \circ E\left(\bar{r}_{X}^{\prime} \otimes \operatorname{id}_{X}\right) \\
& \quad=E\left(\operatorname{id}_{X} \otimes\left(r_{X}^{*} \circ t^{*} \otimes \operatorname{id}_{X}\right)\right) \circ \operatorname{id}_{E(X)} \otimes a_{\bar{X}^{\prime}} \otimes \operatorname{id}_{E(X)} \circ E\left(\left(\operatorname{id}_{X} \otimes t^{*-1} \circ \bar{r}_{X}\right) \otimes \operatorname{id}_{X}\right) \\
& \quad=E\left(\operatorname{id}_{X} \otimes r_{X}^{*}\right) \circ \operatorname{id}_{E(t)} \otimes\left(E\left(t^{*}\right) \circ a_{\bar{X}^{\prime}} \circ E\left(t^{*-1}\right)\right) \otimes \operatorname{id}_{E(X)} \circ E\left(\bar{r}_{X} \otimes \operatorname{id}_{X}\right) \\
& \quad=E\left(\operatorname{id}_{X} \otimes r_{X}^{*}\right) \circ \operatorname{id}_{E(X)} \otimes\left(E\left(t^{*} \circ t^{*-1} \circ a_{\bar{X}}\right) \otimes \operatorname{id}_{E(X)}\right) \circ E\left(\bar{r}_{X} \otimes i_{X}\right) \\
& \quad=E\left(\operatorname{id}_{X} \otimes r_{X}^{*}\right) \circ\left(\operatorname{id}_{E(X)} \otimes a_{\bar{X}} \otimes i_{E(X)}\right) \circ E\left(\bar{r}_{X} \otimes \operatorname{id}_{X}\right),
\end{aligned}
$$

and $S(a)_{X} \in$ End $E(X)$ does not depend on the choice of solution $\left(r_{X}, \bar{r}_{X}, \bar{X}\right)$ of the conjugate equations associated with $X$.

Next we show that $S(a)=\left(S(a)_{X}\right) \in$ Nat $E$ for $a \in A$, i.e., we must show that $E(t) \circ S(a)_{X}=S(a)_{Y} \circ E(t)$, for all $a \in A$ and $t \in \operatorname{Hom}(X, Y)$. By the existence of the transpose $t^{\bullet *} \in \operatorname{Hom}(Y, X)$ given in [23], we get

$$
\begin{aligned}
E(t) \circ S(a)_{X} & =E\left(\operatorname{id}_{Y} \otimes r_{X}^{*}\right) \circ E(t) \otimes a_{\bar{X}} \otimes \operatorname{id}_{E(X)} \circ E\left(\bar{r}_{X} \otimes \operatorname{id}_{X}\right) \\
& =E\left(\operatorname{id}_{Y} \otimes r_{X}^{*}\right) \circ \operatorname{id}_{E(Y)} \otimes a_{\bar{X}} \otimes \operatorname{id}_{E(X)} \circ E\left(\left(t \otimes \operatorname{id}_{\bar{X}} \circ \bar{r}_{X}\right) \otimes \operatorname{id}_{X}\right) \\
& =E\left(\operatorname{id}_{Y} \otimes r_{X}^{*}\right) \circ \operatorname{id}_{E(Y)} \otimes a_{\bar{X}} \otimes \operatorname{id}_{E(X)} \circ E\left(\left(\operatorname{id}_{Y} \otimes t^{\bullet *} \circ \bar{r}_{Y}\right) \otimes \operatorname{id}_{X}\right) \\
& =E\left(\operatorname{id}_{Y} \otimes r_{X}^{*}\right) \circ \operatorname{id}_{E(Y)} \otimes\left(a_{\bar{X}} \circ E\left(t^{\bullet *}\right)\right) \otimes \operatorname{id}_{E(X)} \circ E\left(\bar{r}_{Y} \otimes \operatorname{id}_{X}\right) \\
& =E\left(\operatorname{id}_{Y} \otimes r_{X}^{*}\right) \circ \operatorname{id}_{E(Y)} \otimes\left(E\left(t^{\bullet *}\right) \circ a_{\bar{Y}}\right) \otimes \operatorname{id}_{E(X)} \circ E\left(\bar{r}_{Y} \otimes \operatorname{id}_{X}\right) \\
& =E\left(\operatorname{id}_{Y} \otimes\left(r_{X}^{*} \circ t^{\bullet *} \otimes \operatorname{id}_{X}\right)\right) \circ \operatorname{id}_{E(Y)} \otimes a_{\bar{Y}} \otimes \operatorname{id}_{E(X)} \circ E\left(\bar{r}_{Y} \otimes \operatorname{id}_{X}\right) \\
& =E\left(\operatorname{id}_{Y} \otimes\left(r_{Y}^{*} \circ \operatorname{id}_{\bar{Y}} \otimes t\right)\right) \circ \operatorname{id}_{E(Y)} \otimes a_{\bar{Y}} \otimes \operatorname{id}_{E(X)} \circ E\left(\bar{r}_{Y} \otimes \operatorname{id}_{X}\right) \\
& =E\left(\operatorname{id}_{Y} \otimes r_{Y}^{*}\right) \circ \operatorname{id}_{E(Y)} \otimes a_{\bar{Y}} \otimes E(t) \circ E\left(\bar{r}_{Y} \otimes \operatorname{id}_{Y}\right) \\
& =S(a)_{Y} \circ E(t),
\end{aligned}
$$


as desired.

Clearly, we have $S(a) \in A$ for all $a \in A$ and it is also clear that the map $S$ is linear. We must prove the two identities

$$
m(\iota \otimes S)((a \otimes 1) \Delta(b))=\varepsilon(b) a, \quad m(S \otimes \iota)((1 \otimes a) \Delta(b))=\varepsilon(b) a,
$$

for all $a, b \in A$. We prove only the first one. Thus let $a, b \in A$ and write $(a \otimes 1) \Delta(b)=\sum_{k} a^{k} \otimes b^{k}$ for some $a^{k}, b^{k} \in A$. This means that

$$
\left(a_{X} \otimes 1_{Y}\right) \circ b_{X \otimes Y}=\sum_{k} a_{X}^{k} \otimes b_{Y}^{k}
$$

for all objects $X$ and $Y$ of $\mathcal{C}$. Thus we get

$$
\begin{aligned}
& m(\iota \otimes S)((a \otimes 1) \Delta(b))_{X}=\sum_{k} m\left(a^{k} \otimes S\left(b^{k}\right)\right)_{X}=\sum_{k} a_{X}^{k} \circ S\left(b^{k}\right)_{X} \\
& =\sum_{k} a_{X}^{k} \circ E\left(\operatorname{id}_{X} \otimes r_{X}^{*}\right) \circ \operatorname{id}_{X} \otimes b \frac{k}{X} \otimes \operatorname{id}_{E(X)} \circ E\left(\bar{r}_{X} \otimes \operatorname{id}_{X}\right) \\
& =E\left(\operatorname{id}_{X} \otimes r_{X}^{*}\right) \circ\left(\sum_{k} a_{X}^{k} \otimes b \frac{k}{X}\right) \otimes \operatorname{id}_{E(X)} \circ E\left(\bar{r}_{X} \otimes \operatorname{id}_{X}\right) \\
& =E\left(\operatorname{id}_{X} \otimes r_{X}^{*}\right) \circ\left(a_{X} \otimes \operatorname{id}_{E(\bar{X})} \circ b_{X \otimes \bar{X}}\right) \otimes \operatorname{id}_{E(X)} \circ E\left(\bar{r}_{X} \otimes \operatorname{id}_{X}\right) \\
& =a_{X} \circ E\left(\mathrm{id}_{X} \otimes r_{X}^{*}\right) \circ b_{X \otimes \bar{X}} \otimes \operatorname{id}_{E(X)} \circ E\left(\bar{r}_{X} \otimes \operatorname{id}_{X}\right) \\
& =a_{X} \circ E\left(\mathrm{id}_{X} \otimes r_{X}^{*}\right) \circ E\left(\bar{r}_{X} \otimes \mathrm{id}_{X}\right) b_{1}=a_{X} \circ E\left(\mathrm{id}_{X} \otimes r_{X}^{*} \circ \bar{r}_{X} \otimes \operatorname{id}_{X}\right) b_{1} \\
& =a_{X} \circ E\left(\mathrm{id}_{X}\right) b_{\mathbf{1}}=a_{X} b_{\mathbf{1}}=a_{X} \varepsilon(b)=(\varepsilon(b) a)_{X}
\end{aligned}
$$

for all objects $X$ in $\mathcal{C}$, so $m(\iota \otimes S)((a \otimes 1) \Delta(b))=\varepsilon(b) a$ as natural transformations.

It remains to show that $S$ is invertible. We do that by showing $S\left(S(a)^{*}\right)=a^{*}$ for all $a \in A$. Now with $X \in \mathcal{C}$, we calculate

$$
\begin{aligned}
& S\left(S(a)^{*}\right)_{X}=E\left(\operatorname{id}_{X} \otimes r_{X}^{*}\right) \circ \operatorname{id}_{E(X)} \otimes S(a)_{X}^{*} \otimes \operatorname{id}_{E(X)} \circ E\left(\bar{r}_{X} \otimes \operatorname{id}_{X}\right) \\
= & E\left(\operatorname{id}_{X} \otimes r_{X}^{*}\right) \circ \operatorname{id}_{E(X)} \otimes\left[E\left(\operatorname{id}_{\bar{X}} \otimes \bar{r}_{X}^{*}\right) \circ \operatorname{id}_{E(\bar{X})} \otimes a_{X} \otimes \operatorname{id}_{E(\bar{X})} \circ E\left(r_{X} \otimes \operatorname{id}_{\bar{X}}\right)\right]^{*} \otimes \operatorname{id}_{E(X)} \circ E\left(\bar{r}_{X} \otimes \operatorname{id}_{X}\right),
\end{aligned}
$$

where we used $\left(\bar{r}_{X}, r_{X}, X\right)$ as a solution of the conjugate equations for $\bar{X} \in \mathcal{C}$. Thus

$$
\begin{aligned}
& S\left(S(a)^{*}\right)_{X} \\
& =E\left(\mathrm{id}_{X} \otimes r_{X}^{*}\right) \circ \operatorname{id}_{E(X)} \otimes\left[E\left(r_{X}^{*} \otimes \operatorname{id}_{\bar{X}}\right) \circ \operatorname{id}_{E(\bar{X})} \otimes a_{X}^{*} \otimes \operatorname{id}_{E(\bar{X})} \circ E\left(\operatorname{id}_{\bar{X}} \otimes \bar{r}_{X}\right)\right] \otimes \operatorname{id}_{E(X)} \circ E\left(\bar{r}_{X} \otimes \operatorname{id}_{X}\right) \\
& =E\left(\operatorname{id}_{X} \otimes r_{X}^{*} \circ \operatorname{id}_{X} \otimes r_{X}^{*} \otimes \operatorname{id}_{\bar{X} \otimes X}\right) \circ \operatorname{id}_{E(X \otimes \bar{X})} \otimes a_{X}^{*} \otimes \operatorname{id}_{E(\bar{X} \otimes X)} \circ E\left(\operatorname{id}_{X \otimes \bar{X}} \otimes \bar{r}_{X} \otimes \operatorname{id}_{X} \circ \bar{r}_{X} \otimes \operatorname{id}_{X}\right) \\
& =E\left(\mathrm{id}_{X} \otimes r_{X}^{*} \otimes r_{X}^{*}\right) \circ \mathrm{id}_{E(X \otimes \bar{X})} \otimes a_{X}^{*} \otimes \mathrm{id}_{E(\bar{X} \otimes X)} \circ E\left(\bar{r}_{X} \otimes \bar{r}_{X} \otimes \mathrm{id}_{X}\right) \\
& =E\left(\mathrm{id}_{X} \otimes r_{X}^{*} \otimes r_{X}^{*} \circ \bar{r}_{X} \otimes \mathrm{id}_{X \otimes \bar{X} \otimes X}\right) \circ a_{X}^{*} \otimes \operatorname{id}_{E(\bar{X} \otimes X)} \circ E\left(\bar{r}_{X} \otimes \operatorname{id}_{X}\right) \\
& =E\left(\left(\mathrm{id}_{X} \otimes r_{X}^{*}\right) \circ\left(\bar{r}_{X} \otimes \mathrm{id}_{X}\right) \otimes \mathrm{id}_{\bar{X} \otimes X} \circ \operatorname{id}_{X} \otimes r_{X}^{*}\right) \circ a_{X}^{*} \otimes \operatorname{id}_{E(\bar{X} \otimes X)} \circ E\left(\bar{r}_{X} \otimes \operatorname{id}_{X}\right) \\
& =E\left(\mathrm{id}_{X} \otimes r_{X}^{*}\right) \circ a_{X}^{*} \otimes \operatorname{id}_{E(\bar{X} \otimes X)} \circ E\left(\bar{r}_{X} \otimes \mathrm{id}_{X}\right)=a_{X}^{*} \circ E\left(\mathrm{id}_{X} \otimes r_{X}^{*} \circ \bar{r}_{X} \otimes \operatorname{id}_{X}\right) \\
& =a_{X}^{*} \circ \operatorname{id}_{E(X)}=a_{X}^{*}=\left(a^{*}\right)_{X} \text {. }
\end{aligned}
$$

After these preparatory computations, the following Main Result is essentially a restatement of Theorem 5.21 and Proposition 5.24.

THEOREM 5.25 Let $\mathcal{C}$ be a semisimple tensor $*$-category with conjugates and $E: \mathcal{C} \rightarrow \mathcal{H}$ an embedding functor. Let $(A, \Delta)$ be the corresponding discrete aqg. Then the functor $F: \mathcal{C} \rightarrow \operatorname{Rep}_{f}(A, \Delta)$ defined by

$$
F(X)=\left(E(X), \pi_{X}\right), \quad \pi_{X}(a)=a_{X} \forall a \in A, \quad F(s)=E(s)
$$

provides an equivalence of tensor $*$-categories such that $K \circ F=E$. 
REMARK 5.26 1. In combination with Theorem 3.14 this gives Woronowicz' generalized Tannaka-Krein result. Woronowicz constructs $\operatorname{Corep}_{f}(\hat{A}, \hat{\Delta})$ for a compact aqg $(\hat{A}, \hat{\Delta})$ more directly and uses the universal corepresentation $U$ of $(A, \Delta)$ implicitly. In his proof Woronowicz constructs a compact matrix pseudogroup from a category with a generator. Our result is more general in that this assumption is redundant.

2. In view of Theorem 3.20, an alternative reconstruction theorem for a concrete semisimple tensor $*$-category $\mathcal{C}$ with conjugates would be that

$$
\mathcal{C} \stackrel{\otimes}{\cong} \operatorname{Comod}_{f}\left(\hat{A}, \hat{\Delta}^{o p}\right)
$$

where $\left(\hat{A}, \hat{\Delta}^{o p}\right)$ is a Hopf $*$-algebra with a positive invariant functional. We refer to [34, 15] for similar constructions, where concrete non-semisimple tensor categories are shown to be equivalent to tensor categories of finite dimensional comodules over (infinite dimensional) Hopf algebras (with no *-operation).

We now discuss how the aqg $(A, \Delta)$ depends on the choice of the embedding functor $E$. This discussion is only preliminary and will be taken up again in part II of this series. We need the following

Definition 5.27 Let $\mathcal{C}, \mathcal{D}$ be strict tensor categories and $E, E^{\prime}: \mathcal{C} \rightarrow \mathcal{D}$ be tensor functors (with structural isomorphisms e, $\left.e^{\prime}, d_{X, Y}, d_{X, Y}^{\prime}\right)$. Then $E, E^{\prime}$ are said to be isomorphic as tensor functors, $E \stackrel{\otimes}{\cong} E^{\prime}$, if the diagram

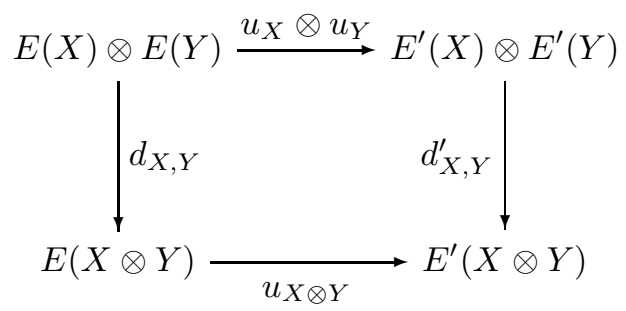

commutes for all $X, Y \in \mathcal{C}$, where $u: E \rightarrow E^{\prime}$ is a natural transformation whose components $u_{X}: E(X) \rightarrow$ $E^{\prime}(X)$ are isomorphisms. A similar and obvious commutative diagram involves the structural isomorphisms $e, e^{\prime}$. If $\mathcal{C}, \mathcal{D}$ are tensor $*$-categories and $E, E^{\prime}$ are $*$-preserving then all $u_{X}$ must be unitary.

Proposition 5.28 Let $\mathcal{C}$ be a semisimple tensor $*$-category $\mathcal{C}$ with conjugates. Let $E, E^{\prime}: \mathcal{C} \rightarrow \mathcal{H}$ be embedding functors, and let $(A, \Delta),\left(A^{\prime}, \Delta^{\prime}\right)$ be the corresponding discrete aqg. An isomorphism $u: E \stackrel{\otimes}{\cong} E^{\prime}$ gives rise to an isomorphism $\theta:(A, \Delta) \cong\left(A^{\prime}, \Delta^{\prime}\right)$ with $\pi_{X}^{\prime} \theta=\pi_{X}$ for all $X \in \mathcal{C}$.

Proof. Let $u: E \rightarrow E^{\prime}$ be a unitary equivalence of tensor $*$-functors. Then $\theta:$ Nat $E \rightarrow$ Nat $E^{\prime}$ given by $\theta(a)_{X}=u_{X} a_{X} u_{X}^{*}$ for $a \in \operatorname{Nat} E$ and $X \in \mathcal{C}$ restricts to a unital $*$-homomorphism from $A$ to $A^{\prime}$. Suppressing as usual the natural transformations $d$ and $d^{\prime}$, we clearly have $\Delta^{\prime}(\theta(a))_{(X, Y)}=u_{X \otimes Y} a_{X \otimes Y} u_{X \otimes Y}^{*}$, whereas $(\theta \otimes \theta) \Delta(a)_{(X, Y)}=\left(u_{X} \otimes u_{Y}\right) a_{X \otimes Y}\left(u_{X}^{*} \otimes u_{Y}^{*}\right)$, for all $a \in \operatorname{Nat} E$ and $X, Y \in \mathcal{C}$. Since $u_{X \otimes Y}=u_{X} \otimes u_{Y}$, for all $X, Y \in \mathcal{C}$, we get $(\theta \otimes \theta) \Delta=\Delta^{\prime} \theta$, and $(A, \Delta) \cong\left(A^{\prime}, \Delta^{\prime}\right)$.

The following definition will be useful in our discussion of embedded symmetric tensor categories.

DeFINITION 5.29 Let $\mathcal{C}, \mathcal{D}$ be tensor categories and $E: \mathcal{C} \rightarrow \mathcal{D}$ a tensor functor. Then the automorphism group $\mathrm{Aut}^{\otimes} E$ consists of the natural isomorphisms of $E$ for which the diagram (5.1) commutes (with $E^{\prime}=E$ ), together with the obvious componentwise group structure. If $\mathcal{C}$ is a tensor $*$-category and $E$ is a $*$-preserving embedding functor then the intrinsic group $G_{E}$ is the subgroup of Aut ${ }^{\otimes} E$ where all $u_{X}$ are unitaries. 


\subsection{The Non- $*$ Case}

Our starting point in this paper is a tensor $*$-category with direct sums, subobjects, conjugates and irreducible unit. From it we (re)construct a multiplier Hopf $*$-algebra with positive left invariant functional. Such algebras were called algebraic quantum groups (aqg) by Van Daele, who now prefers to speak of 'multiplier Hopf *algebras with integrals'. We stick to 'algebraic quantum groups' mainly for the sake of definiteness and brevity. When $A$ is no longer a $*$-algebra and the invariant functional is required to be faithful rather than positive $(\varphi(a b)=0$ for all $a \in A$ implies $b=0)$ we arrive at the notion of a regular multiplier Hopf algebras with left invariant functional. Essentially the entire theory works just as well in this setting, provided one adopts a modified notion of dual. We say a tensor category is rigid if every object $X$ comes with a chosen left dual $\bar{X}$ and morphisms $e_{X}: \bar{X} \otimes X \rightarrow \mathbf{1}$ and $d_{X}: \mathbf{1} \rightarrow X \otimes \bar{X}$ satisfying the triangular equations instead of the conjugate equations. In a semisimple category, left duals are automatically two-sided duals since dual morphisms $e_{X}^{\prime}: \mathbf{1} \rightarrow \bar{X} \otimes X$ and $d_{X}^{\prime}: X \otimes \bar{X} \rightarrow \mathbf{1}$ exist. Adopting the standard definition of semisimple categories as abelian categories with all exact sequences splitting, one can prove the following:

THEOREM 5.30 Let $\mathbb{F}$ be an algebraically closed field (of arbitrary characteristic). Let $\mathcal{C}$ be a $\mathbb{F}$-linear semisimple rigid tensor category with End $\mathbf{1} \cong \mathbb{F}$ and let $E: \mathcal{C} \rightarrow \operatorname{Vect}_{\mathbb{F}}$ be a faithful tensor functor. Then there is a discrete regular multiplier Hopf algebra $(A, \Delta)$ with faithful left invariant functional $\varphi$ and an equivalence $F: \mathcal{C} \rightarrow \operatorname{Rep}(A, \Delta)$ such that $K \circ F=E$.

The proof proceeds exactly as for $*$-categories, ignoring all references to the $*$-preserving property of $E$ and the $*$-involution of $A$. Again, existence, uniqueness and faithfulness of the invariant functional are proven using results due to Van Daele. Only the construction of the coinverse requires careful examination since the notion of dual is different.

We close with some remarks on the two different notions of discreteness applying to multiplier Hopf algebras. In analogy to the $*$-case, a regular multiplier Hopf algebra $(A, \Delta)$ with left invariant functional is called discrete if $A$ is a direct sum of finite dimensional matrix algebras over $\mathbb{F}$. In the non-* case Proposition 2.14 fails, and $(A, \Delta)$ is said to be of discrete type iff there exists $0 \neq h \in A$ such that $a h=\varepsilon(a) h$ for all $a \in A$. As in the *-case, $(A, \Delta)$ is of compact type or, equivalently, compact if $A$ has a unit. It is well known 37] that $(A, \Delta)$ is of discrete type iff $(\hat{A}, \hat{\Delta})$ is of compact type.

Every discrete multiplier Hopf algebra has a copy of $\mathbb{F}$ as a direct summand, corresponding to the onedimensional representation $\varepsilon$. The unit $I_{0}$ of this algebra has the property $a I_{0}=I_{0} a=\varepsilon(a) I_{0}$, thus a discrete multiplier Hopf algebra is of discrete type. The following characterizes discrete multiplier Hopf algebras, generalizing a well known result for finite dimensional Hopf algebras (which automatically possess integrals).

Proposition 5.31 Let $(A, \Delta)$ and $(\hat{A}, \hat{\Delta})$ be mutually dual regular multiplier Hopf algebras with faithful left invariant functionals. Then the following are equivalent:

1. $(A, \Delta)$ is discrete.

2. $(A, \Delta)$ is of discrete type and $h$ can be chosen to be idempotent.

3. $(\hat{A}, \hat{\Delta})$ is of compact type and $\hat{\varphi}(1) \neq 0$.

Proof. The implication 1. $\Rightarrow 2$. has been discussed before. Assuming 2., we have $h=h h=\varepsilon(h) h$, thus $\varepsilon(h)=1$. Conversely, if $\varepsilon(h) \neq 0$ then $h / \varepsilon(h)$ satisfies 2. Now, 2. implies that $(\hat{A}, \hat{\Delta})$ is compact, and compactness implies the existence of $0 \neq h \in A$ satisfying $x h=\varepsilon(x) h$ for all $x \in A$. By definition of the dual $(\hat{A}, \hat{\Delta})$ and assuming the normalization $\varphi(h)=1$, we have $\hat{\varphi}(1)=\varepsilon(h)$, yielding the equivalence $2 . \Leftrightarrow 3$. Now assume 3 . holds. Then $(\hat{A}, \hat{\Delta})$ is a Hopf algebra with left invariant functional $\hat{\varphi}$ satisfying $\hat{\varphi}(1) \neq 0$. By classical results, cf., e.g., 33 , 14.0.3] or [1, Theorem 3.3.2], it follows that $(\hat{A}, \hat{\Delta})$ is cosemisimple. This means that the $\mathbb{F}$-coalgebra $(\hat{A}, \hat{\Delta}, \hat{\varepsilon})$ is a direct sum of finite dimensional coalgebras. Since $\mathbb{F}$ is algebraically closed, every finite dimensional $\mathbb{F}$ coalgebra is a matrix coalgebra, i.e. it has a basis $\left\{a_{i j}, 1 \leq i, j \leq n\right\}$ such that $\Delta\left(a_{i j}\right)=\sum_{l} a_{i l} \otimes a_{l j}$. Since 
$A=\{\hat{\varphi}(\cdot a), a \in \hat{A}\} \subset \hat{A}^{*}$ and $\hat{\varphi}$ is faithful, $A$ has a basis $\left\{b_{i j}^{\alpha}=\hat{\varphi}\left(\cdot a_{i j}^{\alpha}\right), \alpha \in I, 1 \leq i, j \leq n_{\alpha} \in \mathbb{N}\right\}$. These elements multiply according to $b_{i j}^{\alpha} b_{i^{\prime} j^{\prime}}^{\alpha^{\prime}}=\delta_{\alpha \alpha^{\prime}} \delta_{j i^{\prime}} b_{i j^{\prime}}^{\alpha}$, thus

$$
A=\bigoplus_{\alpha \in I} M_{n_{\alpha}}(\mathbb{F})
$$

as desired.

REMARK 5.32 1. In the $*$-case, $\hat{\varphi}$ is positive and $1>0$, thus discrete type implies discreteness.

2. We indicate how Proposition 5.31 can be used to prove Theorem 5.30. Given a semisimple tensor category $\mathcal{C}$ with embedding functor $E$, by 34] there exists a Hopf algebra $H$ such that $\mathcal{C} \simeq \operatorname{Comod} H$. By [32, 14.0.3], semisimplicity of $\mathcal{C}$ implies the existence of a left invariant functional $\hat{\varphi}$ on $H$, satisfying $\hat{\varphi}(1) \neq 0$. Thus $(H, \hat{\Delta})$ is a compact multiplier Hopf algebra with left invariant functional. Its dual $(A, \Delta)$ then is a discrete multiplier Hopf algebra with left invariant functional, and by Theorem 3.14 we have $\mathcal{C} \simeq \operatorname{Rep}_{f}(A, \Delta)$. However, the direct proof of Theorem 5.30 analogous to the proof of Theorem 5.25 is more direct and instructive.

\section{Concrete Braided and Symmetric Categories}

In this section we prove that a braiding for the concrete tensor $*$-category $\mathcal{C}$ gives rise to an $R$-matrix for the associated aqg $(A, \Delta)$. If $\mathcal{C}$ is symmetric and the embedding functor $E$ is symmetric monoidal then we may conclude that $(A, \Delta)$ is cocommutative.

The flip automorphism $\sigma$ of $A \otimes A$ defined by $\sigma(a \otimes b)=b \otimes a$ is a non-degenerate $*$-homomorphism. Thus there exists a unique extension to an involutive $*$-automorphism of $M(A \otimes A)$ which we denote by the same symbol.

Definition 6.1 An aqg $(A, \Delta)$ is said to be quasitriangular w.r.t. $R$ iff $R$ is an invertible element of $M(A \otimes A)$ and satisfies

1. $(\Delta \otimes \iota)(R)=R_{13} R_{23}$,

2. $(\iota \otimes \Delta)(R)=R_{13} R_{12}$,

3. $\Delta^{o p}=R \Delta(\cdot) R^{-1}$,

where $\Delta^{o p} \equiv \sigma \Delta$. We sometimes refer to such an element $R$ as an $R$-matrix. If, in addition, $\sigma(R)=R^{-1}$ we call $(A, \Delta)$ triangular.

The first equation means that the invertible element $R \in M(A \otimes A)$ is a non-degenerate corepresentation of $(A, \Delta)$ on $A$. It is easy to see that $(A, \Delta)$ is quasitriangular w.r.t. $R$ iff $\left(A, \Delta^{o p}\right)$ is quasitriangular w.r.t. $\sigma(R)$.

Definition 6.2 A braiding for a (strict) tensor category $\mathcal{C}$ is a family of isomorphisms $c_{X, Y}: X \otimes Y \rightarrow Y \otimes X$, for all $X, Y \in \mathcal{C}$ natural in both arguments

$$
t \otimes s \circ c_{X, Y}=c_{X^{\prime}, Y^{\prime}} \circ s \otimes t \quad \forall s: X \rightarrow X^{\prime}, t: Y \rightarrow Y^{\prime},
$$

satisfying

$$
\begin{aligned}
c_{X, Y \otimes Z} & =\operatorname{id}_{Y} \otimes c_{X, Z} \circ c_{X, Y} \otimes \mathrm{id}_{Z}, \\
c_{X \otimes Y, Z} & =c_{X, Z} \otimes \mathrm{id}_{Y} \circ \mathrm{id}_{X} \otimes c_{Y, Z}, \\
c_{X, \mathbf{1}} & =c_{\mathbf{1}, X}=\mathrm{id}_{X}
\end{aligned}
$$

for all $X, Y, Z \in \mathcal{C}$. A braiding is called a symmetry if, in addition, it satisfies

$$
c_{X, Y} \circ c_{Y, X}=\operatorname{id}_{Y \otimes X} \quad \forall X, Y \in \mathcal{C} .
$$


A tensor category with a (chosen) braiding or symmetry is called a braided or symmetric tensor category, respectively. A braided functor $F: \mathcal{C} \rightarrow \mathcal{D}$ is a monoidal functor between strict braided tensor categories making

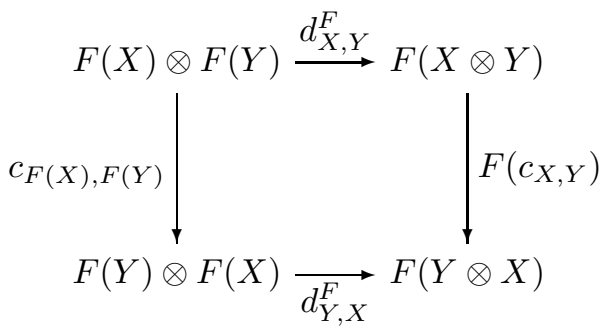

commutative for all $X, Y \in \mathcal{C}$.

The following result establishes the relation between $R$-matrices for $(A, \Delta)$ and braidings for $\operatorname{Rep}_{f}(A, \Delta)$.

Proposition 6.3 Let $(A, \Delta)$ be a discrete aqg. Let $R \in M(A \otimes A)$ and consider

$$
c_{(H, \pi),\left(H^{\prime}, \pi^{\prime}\right)} \equiv \Sigma_{H, H^{\prime}}\left(\overline{\pi \otimes \pi^{\prime}}\right)(R), \quad(H, \pi),\left(H^{\prime}, \pi^{\prime}\right) \in \operatorname{Rep}_{f}(A, \Delta) .
$$

Here $\Sigma_{H, K}$ is the canonical unitary flip from $H \otimes K \rightarrow K \otimes H$. Then the family $\left(c_{(H, \pi),\left(H^{\prime}, \pi^{\prime}\right)}\right)$ is a braiding for $\operatorname{Rep}_{f}(A, \Delta)$ iff $R$ is an R-matrix. Also $c_{(H, \pi),\left(H^{\prime}, \pi^{\prime}\right)}$ is unitary for all $(H, \pi),\left(H^{\prime}, \pi^{\prime}\right)$ iff $R$ is unitary. If these equivalent conditions are satisfied then $(A, \Delta)$ is triangular iff the corresponding braiding for $\operatorname{Rep}_{f}(A, \Delta)$ is symmetric.

Proof. Throughout the proof we write $c_{\pi, \pi^{\prime}}$ for $c_{(H, \pi),\left(H^{\prime}, \pi^{\prime}\right)}$ and note that $c_{\pi, \pi^{\prime}}: H \otimes H^{\prime} \rightarrow H^{\prime} \otimes H$, for all $(H, \pi),\left(H^{\prime} \pi^{\prime}\right) \in \operatorname{Rep}_{f}(A, \Delta)$. Also note that

$$
\overline{\left(\pi \otimes \pi^{\prime}\right)} \sigma(a)=\Sigma_{H^{\prime}, H} \overline{\left(\pi^{\prime} \otimes \pi\right)}(a) \Sigma_{H, H^{\prime}} \quad \forall(H, \pi),\left(H^{\prime}, \pi^{\prime}\right) \in \operatorname{Rep}_{f}(A, \Delta) \quad \forall a \in M(A \otimes A) .
$$

Now suppose $R \in M(A \otimes A)$ satisfies $\Delta^{o p}(\cdot) R=R \Delta(\cdot)$. Then

$$
\begin{aligned}
c_{\pi, \pi^{\prime}}\left(\pi \times \pi^{\prime}\right)(a) & =\Sigma_{H, H^{\prime}}\left(\overline{\pi \otimes \pi^{\prime}}\right)(R)\left(\overline{\pi \otimes \pi^{\prime}}\right) \Delta(a)=\Sigma_{H, H^{\prime}}\left(\overline{\pi \otimes \pi^{\prime}}\right)(R \Delta(a))=\Sigma_{H, H^{\prime}}\left(\overline{\pi \otimes \pi^{\prime}}\right)\left(\Delta^{o p}(a) R\right) \\
& =\Sigma_{H, H^{\prime}}\left(\overline{\pi \otimes \pi^{\prime}}\right) \Delta^{o p}(a)\left(\overline{\pi \otimes \pi^{\prime}}\right)(R)=\Sigma_{H, H^{\prime}}\left(\overline{\pi \otimes \pi^{\prime}}\right)(\sigma \Delta(a)) \Sigma_{H^{\prime}, H} \Sigma_{H, H^{\prime}}\left(\overline{\pi \otimes \pi^{\prime}}\right)(R) \\
& =\left(\overline{\pi^{\prime} \otimes \pi}\right) \Delta(a) \Sigma_{H, H^{\prime}}\left(\overline{\pi \otimes \pi^{\prime}}\right)(R)=\left(\pi^{\prime} \times \pi\right)(a) c_{\pi, \pi^{\prime}},
\end{aligned}
$$

so we have seen that $c_{\pi, \pi^{\prime}}: \pi \times \pi^{\prime} \rightarrow \pi^{\prime} \times \pi$ for all $\pi, \pi^{\prime} \in \operatorname{Rep}_{f}(A, \Delta)$. Conversely, by reversing the above calculations, we see that $\Delta^{o p}(\cdot) R=R \Delta(\cdot)$ if $c_{\pi, \pi^{\prime}}: \pi \times \pi^{\prime} \rightarrow \pi^{\prime} \times \pi$ for all $\pi, \pi^{\prime} \in \operatorname{Rep}_{f}(A, \Delta)$.

It is straightforward to see that $R$ is invertible iff $c_{\pi, \pi^{\prime}}: \pi \times \pi^{\prime} \rightarrow \pi^{\prime} \times \pi$ is an isomorphism for all $\pi, \pi^{\prime} \in \operatorname{Rep}_{f}(A, \Delta)$, that $R$ is unitary iff $c_{\pi, \pi^{\prime}}$ is unitary for all $\pi, \pi^{\prime} \in \operatorname{Rep}_{f}(A, \Delta)$, and that $R^{-1}=\sigma(R)$ iff $c_{\pi^{\prime}, \pi} c_{\pi, \pi^{\prime}}=\mathrm{id}_{\pi, \pi^{\prime}}$ for all $\pi, \pi^{\prime} \in \operatorname{Rep}_{f}(A, \Delta)$.

Pick $\pi, \pi^{\prime}, \pi^{\prime \prime} \in \operatorname{Rep}_{f}(A, \Delta)$. Then

$$
\begin{aligned}
& \overline{\left(\pi \otimes \pi^{\prime} \otimes \pi^{\prime \prime}\right)}(\iota \otimes \Delta)(R)=\overline{\left(\pi \otimes\left(\pi^{\prime} \times \pi^{\prime \prime}\right)\right)}(R) \\
& \quad=\Sigma_{H^{\prime} \otimes H^{\prime \prime}, H} \Sigma_{H, H^{\prime} \otimes H^{\prime \prime}} \overline{\left(\pi \otimes\left(\pi^{\prime} \times \pi^{\prime \prime}\right)\right)}(R)=\Sigma_{H^{\prime} \otimes H^{\prime \prime}, H} c_{\pi, \pi^{\prime} \times \pi^{\prime \prime}},
\end{aligned}
$$

whereas

$$
\begin{aligned}
& \overline{\left(\pi \otimes \pi^{\prime} \otimes \pi^{\prime \prime}\right)}\left(R_{13} R_{12}\right)=\left(\Sigma_{H^{\prime \prime}, H} \Sigma_{H, H^{\prime \prime}} \overline{\left(\pi \otimes \pi^{\prime \prime}\right.}(R)\right)_{13}\left(\Sigma_{H^{\prime}, H} \Sigma_{H, H^{\prime}} \overline{\left(\pi \otimes \pi^{\prime}\right.}(R)\right)_{12} \\
& \quad=\left(\Sigma_{H^{\prime \prime}, H} c_{\pi, \pi^{\prime \prime}}\right)_{13}\left(\Sigma_{H^{\prime}, H} c_{\pi, \pi^{\prime}}\right)_{12}=\left(\Sigma_{H^{\prime \prime}, H}\right)_{13}\left(c_{\pi, \pi^{\prime \prime}}\right)_{13}\left(\Sigma_{H^{\prime}, H}\right)_{12}\left(c_{\pi, \pi^{\prime}}\right)_{12} \\
& \quad=\Sigma_{H^{\prime} \otimes H^{\prime \prime}, H}\left(c_{\pi, \pi^{\prime \prime}}\right)_{23} \quad\left(c_{\pi, \pi^{\prime}}\right)_{12} .
\end{aligned}
$$

Hence $(\iota \otimes \Delta)(R)=R_{13} R_{12}$ iff

$$
c_{\pi, \pi^{\prime} \times \pi^{\prime \prime}}=\left(c_{\pi, \pi^{\prime \prime}}\right)_{23} \quad\left(c_{\pi, \pi^{\prime}}\right)_{12}=\mathrm{id}_{\pi^{\prime}} \otimes c_{\pi, \pi^{\prime \prime}} \circ c_{\pi, \pi^{\prime}} \otimes \mathrm{id}_{\pi^{\prime \prime}}
$$


for all $\pi, \pi^{\prime}, \pi^{\prime \prime} \in \operatorname{Rep}_{f}(A, \Delta)$. Similarly, one shows that $(\Delta \otimes \iota) R=R_{13} R_{23}$ iff

$$
c_{\pi \times \pi^{\prime}, \pi^{\prime \prime}}=\left(c_{\pi, \pi^{\prime \prime}}\right)_{12} \quad\left(c_{\pi^{\prime}, \pi^{\prime \prime}}\right)_{23}=c_{\pi, \pi^{\prime \prime}} \otimes \mathrm{id}_{\pi^{\prime}} \circ \mathrm{id}_{\pi} \otimes c_{\pi^{\prime}, \pi^{\prime \prime}},
$$

for all $\pi, \pi^{\prime}, \pi^{\prime \prime} \in \operatorname{Rep}_{f}(A, \Delta)$.

TheOREM 6.4 Let $\mathcal{C}$ be a semisimple braided tensor $*$-category with conjugates and $E: \mathcal{C} \rightarrow \mathcal{H}$ a faithful $*$ preserving tensor functor. Let $(A, \Delta)$ and $F: \mathcal{C} \rightarrow \operatorname{Rep}_{f}(A, \Delta)$ be as constructed in Theorem 5.2S. Then $(A, \Delta)$ is quasitriangular w.r.t. a unique $R \in M(A \otimes A)$ such that the functor $F: \mathcal{C} \rightarrow \operatorname{Rep}_{f}(A, \Delta)$ is an equivalence of braided tensor categories w.r.t. the braiding for $\operatorname{Rep}_{f}(A, \Delta)$ provided by $R$. $R$ is unitary iff $c_{X, Y}$ is unitary for all $X, Y .(A, \Delta)$ is triangular iff the corresponding braiding for $\operatorname{Rep}_{f}(A, \Delta)$ is symmetric.

Proof. For $i, j \in I_{\mathcal{C}}$ we have

$$
F\left(c_{X_{i}, X_{j}}\right) \in \operatorname{Mor}_{\mathcal{H}}\left(E\left(X_{i}\right) \otimes E\left(X_{j}\right), E\left(X_{j}\right) \otimes E\left(X_{i}\right)\right)=B\left(E\left(X_{i}\right) \otimes E\left(X_{j}\right), E\left(X_{j}\right) \otimes E\left(X_{i}\right)\right) .
$$

Thus $\Sigma_{E\left(X_{j}\right), E\left(X_{i}\right)} F\left(c_{X_{i}, X_{j}}\right) \in B\left(E\left(X_{i}\right) \otimes E\left(X_{j}\right)\right)$ and we can define $R \in M(A \otimes A)=\prod_{i j} B\left(E\left(X_{i}\right) \otimes E\left(X_{j}\right)\right)$ by $R_{i j}=\Sigma_{E\left(X_{j}\right), E\left(X_{i}\right)} F\left(c_{X_{i}, X_{j}}\right)$. Naturality of the braiding and functoriality of $F$ now imply that we have

$$
F\left(c_{X, Y}\right)=\Sigma_{E(X), E(Y)}\left(\overline{\pi_{X} \otimes \pi_{Y}}\right)(R) \quad \forall X, Y \in \mathcal{C} .
$$

Since $F$ is an equivalence, we see that the family $F\left(c_{X, Y}\right)$ is a braiding for $\operatorname{Rep}_{f}(A, \Delta)$. Denote this family by $\left(c_{\left(E(X), \pi_{X}\right),\left(E(Y), \pi_{Y}\right)}\right)$, so

$$
c_{\left(E(X), \pi_{X}\right),\left(E(Y), \pi_{Y}\right)}=\Sigma_{E(X), E(Y)}\left(\overline{\pi_{X} \otimes \pi_{Y}}\right)(R) \quad \forall X, Y \in \mathcal{C} .
$$

By Proposition 6.3, the element $R \in M(A \otimes A)$ is therefore an $R$-matrix which is unitary iff $c_{X, Y}$ is unitary for all $X, Y$ and satisfies $\sigma(R)=R^{-1}$ iff $c_{Y, X} \circ c_{X, Y}=\operatorname{id}_{X \otimes Y}$ for all $X, Y$. With the braiding on $\operatorname{Rep}_{f}(A, \Delta)$ given by $R$ according to Proposition 6.3, we get by definition $F\left(c_{X, Y}\right)=c_{F(X), F(Y)}$, for all $X, Y$, as $F(X)=\left(E(X), \pi_{X}\right)$ for all $X$. Thus $F$ is a braided functor.

REMARK 6.5 It seems worthwhile comparing our result with [16, Proposition XIII.1.4], where it is shown that the tensor category of left modules over a bialgebra $(H, m, \eta, \Delta, \varepsilon)$ is braided iff $H$ is quasitriangular w.r.t. some $R$-matrix. The proof uses the left regular representation of $H$ and therefore would not apply to the category of finite dimensional $H$-modules if $H$ is infinite dimensional. Our setting differs too in that $A$ is non-unital whenever it is infinite dimensional.

REMARK 6.6 As is well known, the category $\mathcal{H}$ of Hilbert spaces has a unique braiding, the flip $\Sigma_{H, K}: H \otimes K \rightarrow$ $K \otimes H$. It is thus natural to ask when the functors $E$ respects this braiding. If so, then

$$
E\left(c_{X, Y} \circ c_{Y, X}\right)=E\left(c_{X, Y}\right) \circ E\left(c_{Y, X}\right)=\Sigma_{E(X), E(Y)} \circ \Sigma_{E(Y), E(X)}=\operatorname{id}_{E(Y) \otimes E(X)}=\operatorname{id}_{E(Y \otimes X)}=E\left(\operatorname{id}_{Y \otimes X}\right),
$$

where we suppressed the isomorphisms $d_{X, Y}^{E}$ as is our policy throughout. If $E$ is faithful this implies $c_{X, Y} \circ$ $c_{Y, X}=\operatorname{id}_{Y \otimes X}$, thus $\mathcal{C}$ is symmetric. In other words, for non-symmetric categories $\mathcal{C}$ there is no embedding functor satisfying $E\left(c_{X, Y}\right)=\Sigma_{E(X), E(Y)}$. For symmetric $\mathcal{C}$ we have the following easy yet important result.

Corollary 6.7 Let $\mathcal{C}$ be a semisimple symmetric tensor $*$-category and let $E: \mathcal{C} \rightarrow \mathcal{H}$ be a symmetric embedding functor, i.e., $E\left(c_{X, Y}\right)=\Sigma_{E(X), E(Y)}$ for all $X, Y \in \mathcal{C}$. Then the corresponding discrete aqg $(A, \Delta)$ is cocommutative and $F$ is a $*$-preserving symmetric monoidal equivalence. 
Proof. It is clear from the definition of the $R$-matrix $R$ in the proof of Theorem 6.4 that $R=1$ in this case, so $\Delta^{o p}=\Delta$.

We are now in a position to re-prove the classical Tannaka-Krein duality theorem for compact groups.

THEOREM 6.8 Let $\mathcal{C}$ be a semisimple tensor $*$-category with conjugates and unitary symmetry, and let $E: \mathcal{C} \rightarrow$ $\mathcal{H}$ be $a *$-preserving faithful symmetric tensor functor. Then there exists a compact group $G$ and an equivalence $F^{\prime}: \mathcal{C} \rightarrow \operatorname{Rep}_{f} G$ of symmetric tensor $*$-categories such that $K^{\prime} \circ F^{\prime}=E$, where $K^{\prime}: \operatorname{Rep}_{f} G \rightarrow \mathcal{H}$ is the forgetful functor. The group $G$ is canonically isomorphic to the intrinsic group $G_{E}$ of the embedding functor.

Proof. Under the given assumptions, Theorem 6.4 provides us with a discrete aqg $(A, \Delta)$ and an equivalence $F: \mathcal{C} \rightarrow \operatorname{Rep}_{f}(A, \Delta)$ of tensor $*$-categories such that $K \circ F=E$. By assumption, $E$ preserves the symmetries, i.e. $E\left(c_{X, Y}\right)=\Sigma_{E(X), E(Y)}$ for all $X, Y \in \mathcal{C}$. Then by Corollary 6.7 $(A, \Delta)$ is cocommutative. Let $G$ be the intrinsic group of $(A, \Delta)$ as defined in Definition 4.1. By Theorem 4.11 we have a (concretely given) equivalence $D: \operatorname{Rep}_{f}(A, \Delta) \rightarrow \operatorname{Rep}_{f} G$ of tensor $*$-categories. Thus the composition $F^{\prime}=D \circ F: \mathcal{C} \rightarrow \operatorname{Rep} f G$ is the $\operatorname{desired}$ equivalence of tensor $*$-categories. It satisfies $K^{\prime} \circ F^{\prime}=K^{\prime} \circ D \circ F=K \circ F=E$. The final claim is obvious, since the unitary grouplike elements of $(A, \Delta)$ are by definition of $(A, \Delta)$ precisely the unitary natural isomorphisms of $E$ that are compatible with the tensor structures, in the sense of (5.1).

We end this section with some general facts on $R$-matrices for quasitriangular aqg.

Proposition 6.9 Let $(A, \Delta)$ be a quasitriangular aqg w.r.t. R. Then the following statements hold:

1. $R_{12} R_{13} R_{23}=R_{23} R_{13} R_{12}$.

2. $(\varepsilon \otimes \iota) R=1=(\iota \otimes \varepsilon) R$.

3. $(S \otimes \iota) R=R^{-1}=\left(\iota \otimes S^{-1}\right)(R)$.

4. $(S \otimes S) R=R$.

The first equation is called the 'quantum Yang-Baxter equation'.

Proof. $R$ is a non-degenerate corepresentation of $(A, \Delta)$ on $A$, so 2 . and the first equation in 3 . follow immediately. The second equation in 3. thus follows by noting that $\left(A, \Delta^{o p}\right)$ is quasitriangular w.r.t. $\sigma(R)$ with coinverse $S^{-1}$. Claim 4 follows from 3. by $(S \otimes S) R=(\iota \otimes S)(S \otimes \iota) R=(\iota \otimes S)\left(R^{-1}\right)=(\iota \otimes S)\left(\iota \otimes S^{-1}\right) R=R$. Finally, to prove 1 . first note that both the maps $\Delta^{o p} \otimes \iota$ and $R_{12}(\Delta \otimes \iota)(\cdot) R_{12}^{-1}$ are non-degenerate homomorphisms from $A \otimes A$ to $M(A \otimes A \otimes A)$, and thus both have unique extensions to $M(A \otimes A)$. Because $\Delta^{o p}=R \Delta(\cdot) R^{-1}$ these maps clearly coincide on $A \otimes A$, and thus on $M(A \otimes A)$, extensions being unique. Hence

$$
R_{12} R_{13} R_{23} R_{12}^{-1}=R_{12}(\Delta \otimes \iota)(R) R_{12}^{-1}=\left(\Delta^{o p} \otimes \iota\right)(R)=(\sigma \otimes \iota)(\Delta \otimes \iota)(R)=(\sigma \otimes \iota)\left(R_{13} R_{23}\right)=R_{23} R_{13}
$$

as desired.

Proposition 6.10 Suppose $(A, \Delta)$ is a quasitriangular aqg w.r.t. $R$. Let $U$ denote the universal corepresentation of $(A, \Delta)$ as defined in Theorem 3.4. Define $\pi_{R}: \hat{A} \rightarrow M(A)$ as in Proposition 3.5. Then the following hold:

1. $\pi_{R}$ is a non-degenerate homomorphism.

2. $\left(\pi_{R} \otimes \pi_{R}\right) \hat{\Delta}=\Delta^{o p} \pi_{R}$.

3. $\Delta^{o p}(\cdot)\left(\iota \otimes \pi_{R}\right) U=\left(\iota \otimes \pi_{R}\right) U \Delta(\cdot)$.

Conversely, any map $\pi: \hat{A} \rightarrow M(A)$ satisfying these three properties arises from a unique $R \in M(A \otimes A)$ with $\pi=\pi_{R}$ making $(A, \Delta)$ into a quasitriangular aqg. Moreover, the map $\pi_{R}$ is *-preserving iff $R$ is unitary. 
Proof. It suffices to show that statement 2 is equivalent to $(\iota \otimes \Delta)(R)=R_{13} R_{12}$, this being the only non-trivial step not covered by Proposition 3.5 and Theorem 3.6. But by Theorem 3.4, we get

$$
\begin{aligned}
& \left(\pi_{R} \otimes \pi_{R}\right) \hat{\Delta} \omega=\left(\pi_{R} \otimes \pi_{R}\right) \hat{\Delta}(\omega \otimes \iota) U=\left(\pi_{R} \otimes \pi_{R}\right)(\omega \otimes \iota \otimes \iota)(\iota \otimes \hat{\Delta}) U=\left(\pi_{R} \otimes \pi_{R}\right)(\omega \otimes \iota \otimes \iota)\left(U_{12} U_{13}\right) \\
& \left.\quad=(\omega \otimes \iota \otimes \iota)\left(\iota \otimes \pi_{R} \otimes \pi_{R}\right)\left(U_{12} U_{13}\right)=(\omega \otimes \iota \otimes \iota)\left[\left(\iota \otimes \pi_{R}\right) U\right)_{12}\left(\left(\iota \otimes \pi_{R}\right) U\right)_{13}\right]=(\omega \otimes \iota \otimes \iota)\left(R_{12} R_{13}\right)
\end{aligned}
$$

for all $\omega \in \hat{A}$, whereas

$$
\Delta^{o p} \pi_{R}(\omega)=\Delta^{o p}(\omega \otimes \iota) R=(\omega \otimes \iota \otimes \iota)\left(\iota \otimes \Delta^{o p}\right) R
$$

for all $\omega \in \hat{A}$.

Note that 1. and 2. just mean that $\pi_{R}$ is a morphism of multiplier Hopf algebras from $(\hat{A}, \hat{\Delta})$ to $\left(A, \Delta^{o p}\right)$. It is even an aqg morphism iff $R$ is unitary by the last statement of the proposition.

In Definition 3.13 we introduced the tensor product $V \times V^{\prime}=V_{12} V_{13}^{\prime} \in M\left(A \otimes B \otimes B^{\prime}\right)$ of two unitary corepresentations $V$ and $V^{\prime}$ on $B$ and $B^{\prime}$, respectively. Another choice of a tensor product which works equally well, is given by $V \times_{o p} V^{\prime}=V_{13}^{\prime} V_{12} \in M\left(A \otimes B \otimes B^{\prime}\right)$. In general there is no relation between these two tensor products. In view of the relation between representations of an aqg and corepresentations of its dual aqg (and the relation between the identity representation and the universal corepresentation), the following result should not come as a big surprise.

Proposition 6.11 Let $(A, \Delta)$ be an aqg with universal corepresentation $U$, so hat $U=\sigma(U)$ is the universal corepresentation of $(\hat{A}, \hat{\Delta})$ on $A$. Let $R$ be any element of $M(A \otimes A)$. Then $\Delta^{o p}(\cdot) R=R \Delta(\cdot)$ iff

$$
\left(\hat{U} \times_{o p} \hat{U}\right) R_{23}=R_{23}(\hat{U} \times \hat{U}) .
$$

Proof. By Theorem 3.4, we get

$$
\begin{gathered}
\Delta^{o p}(a) R=\left(\Delta^{o p}(\iota \otimes a) U\right) R=(\sigma \Delta(\iota \otimes a) U) R \\
=((\sigma \otimes a)(\Delta \otimes \iota) U) R=\left((\iota \otimes \iota \otimes a)(\sigma \otimes \iota)\left(U_{13} U_{23}\right)\right) R=(\iota \otimes \iota \otimes a)\left(U_{23} U_{13} R_{12}\right)
\end{gathered}
$$

for all $a \in A$, whereas

$$
R \Delta(a)=R \Delta(\iota \otimes a) U=R(\iota \otimes \iota \otimes a)(\Delta \otimes \iota) U=R(\iota \otimes \iota \otimes a)\left(U_{13} U_{23}\right)=(\iota \otimes \iota \otimes a)\left(R_{12} U_{13} U_{23}\right)
$$

for all $a \in A$. Hence $\Delta^{o p}(\cdot) R=R \Delta(\cdot)$ iff $U_{23} U_{13} R_{12}=R_{12} U_{13} U_{23}$. Permuting indices, we see that the latter equation is equivalent to $U_{31} U_{21} R_{23}=R_{23} U_{21} U_{31}$, which again is equivalent to $\hat{U}_{13} \hat{U}_{12} R_{23}=R_{23} \hat{U}_{12} \hat{U}_{13}$ as desired.

So given any unitary $R$-matrix $R$, the map $f \in \operatorname{Hom}(A \otimes A, A \otimes A)$ given by $f(b)=R b R^{*}$, for all $b \in A \otimes A$, is an arrow from $\Delta$ to $\Delta^{o p}$ and from $\hat{U} \times \hat{U}$ to $\hat{U} \times_{o p} \hat{U}$ in the sense of Remark 3.9. Also $\delta_{R}(a)=f(1 \otimes a)$ for $a \in A$, is a coaction in the sense of Remark 3.10. Similarly, the linear map $\delta_{R}^{\prime}: A \rightarrow M(A \otimes A)$ given by $\delta_{R}^{\prime}(a)=f(a \otimes 1)$, for all $a \in A$, is a non-degenerate $*$-homomorphism such that $\left(\delta_{R}^{\prime} \otimes \iota\right) \delta_{R}^{\prime}=\left(\iota \otimes \Delta^{o p}\right) \delta_{R}^{\prime}$ and $(\iota \otimes \varepsilon) \delta_{R}^{\prime}=\iota$.

\section{References}

[1] E. Abe: Hopf Algebras. Cambridge University Press, 1977.

[2] J. Barrett \& B. Westbury: Spherical categories. Adv. Math. 143, 357-375 (1999).

[3] E. Bedos, R. Conti \& L. Tuset: On amenability and co-amenability of algebraic quantum groups and their corepresentations. math.0A/0111027. 
[4] E. Bedos \& L. Tuset: Amenability of locally compact quantum groups. Preprint (2001).

[5] E. Bedos, G. J. Murphy \& L. Tuset: Co-amenability of compact quantum groups. Journ. Geom. Phys. 40, 130-153 (2001).

[6] L. Breen: Tannakian categories. Proc. Symp. Pure Math. 55, 337-376 (1994).

[7] P. Deligne \& J. S. Milne: Tannakian categories. Lecture notes in mathematics 900, 101-228. Springer 1982.

[8] P. Deligne: Catégories tannakiennes. In: P. Cartier et al. (eds.): Grothendieck Festschrift, vol. II, pp. 111-195. Birkhäuser Verlag 1991.

[9] S. Doplicher \& J. E. Roberts: A new duality theory for compact groups. Invent. Math. 98, 157-218 (1989).

[10] S. Doplicher, C. Pinzari \& J. E. Roberts: An algebraic duality theory for multiplicative unitaries. Internat. J. Math. 12, 415-459 (2001).

[11] M. Enock \& J. M. Schwartz: Kac Algebras and Duality of Locally Compact Groups. Springer Verlag, 1980.

[12] P. Ghez, R. Lima \& J. E. Roberts: $W^{*}$-Categories. Pac. Journ. Math. 12, 79-109 (1985).

[13] D. Guido \& L. Tuset: Representations of direct products of matrix algebras. Fund. Math. 169, 145-160 (2001).

[14] E. Hewitt \& K. A. Ross: Abstract Harmonic Analysis I 83 II. 2nd ed., Springer Verlag, 1994.

[15] A. Joyal \& R. Street: An introduction to Tannaka duality and quantum groups. In: Proceedings of the conference on category theory, Como 1990. Lecture notes in mathematics 1488, 413-492 (1991).

[16] C. Kassel: Quantum Groups. Springer Verlag, 1995.

[17] A. Klimyk \& K. Schmüdgen: Quantum Groups and their Representations. Springer Verlag, 1997.

[18] J. Kustermans: Examining the dual of an algebraic quantum group. funct-an/9704004.

[19] J. Kustermans: Locally compact quantum groups in the universal setting. Internat. J. Math. 12, 289-338 (2001).

[20] J. Kustermans \& A. Van Daele: $\mathrm{C}^{*}$-algebraic quantum groups arising from algebraic quantum groups. Internat. J. Math. 8, 1067-1139 (1997).

[21] Kustermans, J. and Vaes, S.: Locally Compact Quantum Groups. Ann. Scient. Ec. Norm. Sup. 33, 837-934 (2000).

[22] Kustermans, J. and Vaes, S.: Locally Compact Quantum Groups in the von Neumann algebraic setting. math.QA/0005219.

[23] R. Longo \& J. E. Roberts: A theory of dimension. K-Theory 11, 103-159 (1997).

[24] S. Mac Lane: Categories for the Working Mathematician. 2nd edition. Springer, 1997.

[25] M. Müger: Galois theory for braided tensor categories and the modular closure. Adv. Math. 150, 151-201 (2000).

[26] M. Müger: From subfactors to categories and topology I. Frobenius algebras in and Morita equivalence of tensor categories. math.CT/0111204.

[27] G. Pedersen: Analysis Now. Springer Verlag, 1989.

[28] P. Podlés \& S. L. Woronowicz: Quantum deformation of Lorentz group. Commun. Math. Phys. 130, 381-431 (1990). 
[29] J. E. Roberts: The reconstruction problem. Unpublished notes.

[30] J. E. Roberts \& L. Tuset: On the equality of $q$-dimensions and intrinsic dimension. J. Pure Appl. Alg. 156, 329-343 (2001).

[31] N. Saavedra Rivano: Catégories Tannakiennes. Lecture notes in mathematics 265. Springer Verlag, 1972.

[32] M. E. Sweedler: Hopf Algebras. Benjamin, 1969.

[33] T. Tannaka: Über den Dualitätssatz der nichtkommutativen topologischen Gruppen. Tôhoku Math. Journ. 45, 1-12 (1939).

[34] K.-H. Ulbrich: On Hopf algebras and rigid monoidal categories. Israel Math. J. 72, 252-256 (1990).

[35] A. Van Daele: Multiplier Hopf algebras. Trans. Amer. Math. Soc. 342, 917-932 (1994).

[36] A. Van Daele: Discrete quantum groups. Journ. Alg. 180, 431-444 (1996).

[37] A. Van Daele: An algebraic framework for group duality. Adv. in Math. 140, 323-366 (1998).

[38] A. Van Daele: The Haar measure on some locally compact quantum groups. math.0A/0109004.

[39] A. Van Daele \& Y. Zhang: Corepresentation theory of multiplier Hopf algebra I. Internat. Journ. Math. 10, 503-539 (1999).

[40] S. L. Woronowicz: Compact matrix pseudogroups. Commun. Math. Phys. 111, 613-665 (1987).

[41] S. L. Woronowicz: Tannaka-Krein duality for compact matrix pseudogroups. Invent. Math. 93, 35-76 (1988).

[42] S. L. Woronowicz: Compact quantum groups. In: A. Connes, K. Gawedzki \& J. Zinn-Justin (eds.): Symétries Quantiques. [Quantum Symmetries]. Proceedings of the NATO Advanced Study Institute Summer School in Theoretical Physics in Les Houches, 1995. North-Holland, 1998.

[43] S. Yamagami: On unitary representation theories of compact quantum groups. Commun. Math. Phys. 167, 509-529 (1995).

[44] S. Yamagami: Frobenius duality in $C^{*}$-tensor categories. Preprint, 2000.

[45] D. N. Yetter: Quantum groups and representations of monoidal categories. Math. Proc. Camb. Phil. Soc. 108, 261-290 (1990).

Addresses of the authors:

Michael Müger, Korteweg-de Vries Instituut, Universiteit van Amsterdam, Amsterdam, Netherlands.

E-mail: mmueger@science.uva.nl.

John E. Roberts, Dipartimento di Matematica, Università di Roma II 'Tor Vergata', Roma, Italy.

E-mail: roberts@mat.uniroma2.it.

Lars Tuset, Faculty of Engineering, Oslo University College, Oslo, Norway.

E-mail: Lars.Tuset@iu.hio.no. 